\title{
Complementary Synthetic Approaches Toward 9-Phosphatriptycene and Structure-Reactivity Investigations of its Association with Sterically \\ Hindered Lewis Acids
}

Lei Hu, ${ }^{\S \ddagger}$ Damien Mahaut, ${ }^{\S}$ Nikolay Tumanov, ${ }^{\S}$ Johan Wouters, ${ }^{\S}$ Raphaël Robiette, ${ }^{*,+}$

Guillaume Berionni*,§

$\S$ University of Namur, Department of Chemistry, Namur Institute of Structured Matter. Rue de Bruxelles 61, 5000 Namur, Belgium

† Université catholique de Louvain, Institute of Condensed Matter and Nanosciences, Place Louis Pasteur 1 box L4.01.02, 1348 Louvain-la-Neuve, Belgium

Table of Contents

1. Determination of Tolman electronic parameter and of the Lewis basicity of $\mathbf{1}$ Page S2

2. Copies of NMR and mass spectra

Page S6

3. Single-crystal X-ray diffraction analysis

Page S17

4. Computational studies

Page S25

5. References

Page S53 


\section{Determination of Tolman electronic parameter and of the Lewis basicity of 1.}

1.1 IR measurements of the $v_{\text {co }}$ stretching frequency in the $\mathrm{Rh}(\mathrm{acac}) \mathrm{CO}(9$-phosphatriptycene) complex.

$\mathrm{Rh}(\mathrm{acac})(\mathrm{CO})\left(\mathrm{PR}_{3}\right)$ complexes were prepared by mixing a phosphine $\mathrm{PR}_{3}$ and $\mathrm{Rh}(\mathrm{CO})_{2} \mathrm{acac}$ under stoichiometric amounts in $0.5 \mathrm{~mL}$ of $\mathrm{CH}_{2} \mathrm{Cl}_{2}$ in a glove-box and the solution was placed in a IR quartz cell. A linear correlation between the $v$ co stretching frequencies of $\mathrm{Rh}(\mathrm{acac})(\mathrm{CO})\left(\mathrm{PR}_{3}\right)$ and the vco stretching frequencies of $\mathrm{Ni}(\mathrm{CO})_{3}\left(\mathrm{PR}_{3}\right)$ complexes used originally for determining the Tolman electronic parameter TEP has previously been reported by Carow, ${ }^{1}$ which allowed us to determine the TEP value for 9phosphatriptycene 1. We also checked that our IR measurements of the vCO stretching frequency in several $\mathrm{Rh}(\mathrm{acac})(\mathrm{CO})\left(\mathrm{PR}_{3}\right)$ complexes were in agreement with literature data and found that our results are within $\mathrm{a} \pm 2 \mathrm{~cm}^{-1}$ range from reported values (Table S1).

Table S1: Infrared stretching frequencies $\left(\mathrm{cm}^{-1}\right)$ and Tolman electronic parameter for representative phosphines.

\begin{tabular}{lccc}
\hline Phosphines & $\begin{array}{c}\text { TEP } \\
/ \mathbf{c m}^{-1}\end{array}$ & $\begin{array}{c}\text { V(CO) } \mathbf{R h} \text { complex } \\
/ \mathbf{c m}^{\mathbf{1}}\end{array}$ & $\begin{array}{c}\boldsymbol{V}(\mathbf{C O}) \mathbf{R h} \text { complex } \\
/ \mathbf{c m}^{-\mathbf{1}}(\text { this work })\end{array}$ \\
\hline $\mathrm{POPh}_{3}$ & 2085 & 2008 & 2007 \\
$\mathrm{POEt}_{3}$ & 2077 & not reported & 1989 \\
$\mathrm{P}-$ triptycene $\mathbf{1}$ & 2076 & not reported & 1985 \\
$\mathrm{P}\left(p-\mathrm{CF}_{3} \mathrm{C}_{6} \mathrm{H}_{5}\right)_{3}$ & - & 1986 & not determined \\
$\mathrm{P}\left(p-\mathrm{ClC}_{6} \mathrm{H}_{5}\right)_{3}$ & 2073 & 1982 & 1980 \\
$\mathrm{PPh}_{3}$ & 2069 & 1978 & 1976 \\
$\mathrm{P}(p-\text { tolyl })_{3}$ & 2067 & 1974 & 1974 \\
$\mathrm{P} t \mathrm{Bu}_{3}$ & 2056 & 1956 & 1958 \\
\hline
\end{tabular}

${ }^{\mathrm{a}}$ From references 2 and 3 .

1.2 Determination of the Lewis basicity parameter $L B$ of 9-phosphatriptycene 1 from the reversible reaction with benzhydrylium tetrafluoroborates $\mathbf{1 2 a}-\mathbf{b}$ in $\mathrm{CD}_{3} \mathrm{CN}$.

The reaction of 9-phosphatriptycene $1(10.0 \mathrm{mg}, 0.037 \mathrm{mmol}, 0.041 \mathrm{M})$ with the weakly acidic $(L A=-$ $6.33)^{4}$ benzhydrylium ion 12a $(13.4 \mathrm{mg}, 0.037 \mathrm{mmol}, 0.041 \mathrm{M})$ does not proceed in $\mathrm{CD}_{3} \mathrm{CN}(0.6 \mathrm{~mL})$ at $23{ }^{\circ} \mathrm{C}$ in $48 \mathrm{~h}$.

The reaction of 9-phosphatriptycene $1(10.0 \mathrm{mg}, 0.037 \mathrm{mmol}, 0.041 \mathrm{M})$ with the strongly acidic $(L A=-$ $0.83)^{4}$ benzhydrylium ion 12b $(17.5 \mathrm{mg}, 0.037 \mathrm{mmol}, 0.041 \mathrm{M})$ proceeded spontaneously in $\mathrm{CD}_{3} \mathrm{CN}(0.6$ $\mathrm{mL}$ ) at $23{ }^{\circ} \mathrm{C}$ with an immediate color change from dark blue to light pink. The association is reversible because the characteristic signals of the uncomplexed $\mathbf{1}$ and $\mathbf{1 2} \mathrm{b}$ and of the Lewis adduct 13b are observed simultaneously in the ${ }^{1} \mathrm{H}$ and ${ }^{31} \mathrm{P}$ NMR spectra (see section 2.4 page $\mathrm{S} 12$ ).

From the ${ }^{1} \mathrm{H}$ NMR integrals of the representative proton underline on the spectrum below, the equilibrium constant $K_{\text {eq }}$ can be determined. From this value, the Lewis basicity $L B$ of $\mathbf{1}$ is calculated by the equations below and with the reported $L A$ parameter of $\mathbf{1 2 b}(L A=-6.33) .{ }^{4}$

$$
\begin{gathered}
\log K_{\mathrm{eq}}=\log \frac{\left[\text { Lewis adduct 13] }\left[\mathrm{BF}_{4}^{-}\right]\right.}{[\text {free cation 12] }[\text { free Ptrip 1] }}=L A+L B \\
K_{\text {eq }}=(1.04)^{2} /(0.36 \times 0.15)=20.03 \\
L B=\log K_{\text {eq }}-L A=7.63
\end{gathered}
$$




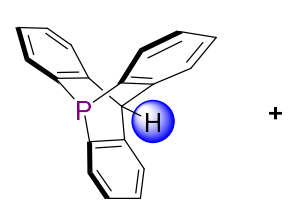

1

${ }^{1} \mathrm{H}$ NMR: 5.75 ppm

(integral $=0.15$ )

${ }^{31}$ P NMR: -64.4 ppm

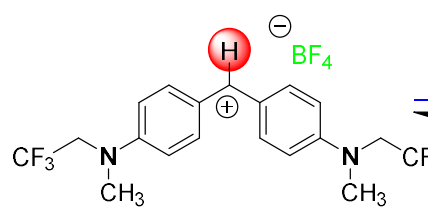

$(\mathrm{mfa})_{2} \mathrm{CH}^{+} \mathrm{BF}_{4}^{-12 b}$

${ }^{1} \mathrm{H}$ NMR: 8.4 ppm

(integral $=0.36$ )

LA: -6.33

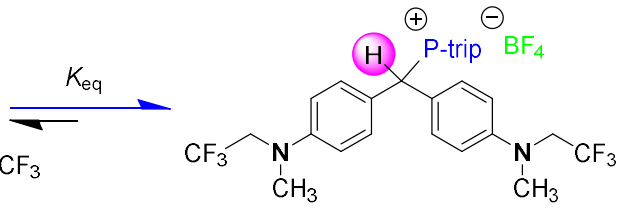

Lewis adduct $13 \mathrm{~b}$ ${ }^{1} \mathrm{H}$ NMR: 6.25 ppm (integral $=1.04$ ) ${ }^{31}$ P NMR: -5.5 ppm

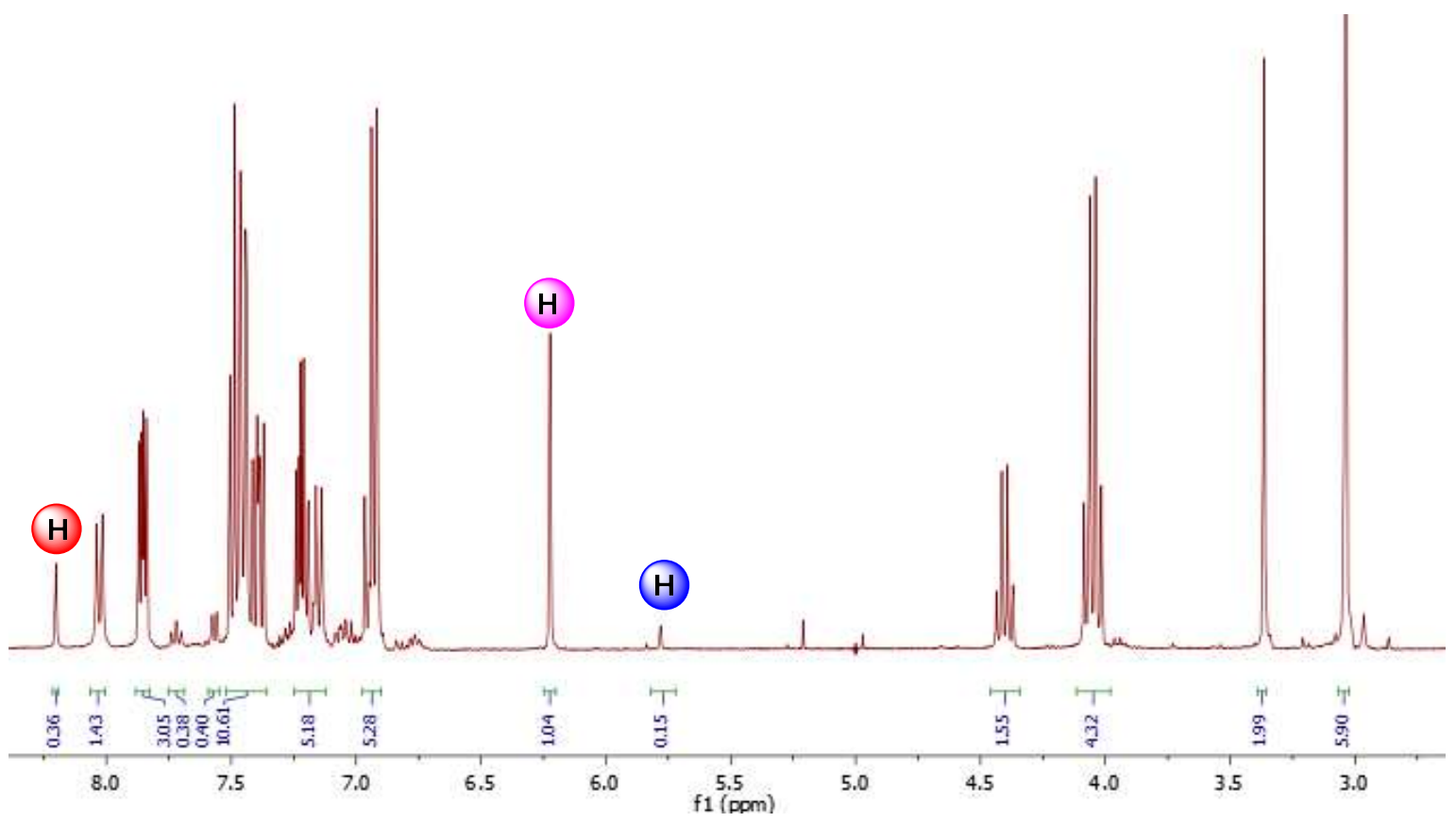

1.3 Determination of the association constant $K$ between $\mathrm{B}\left(\mathrm{C}_{6} \mathrm{~F}_{5}\right)_{3}$ and $\mathbf{1}$ by UV-Vis titrations.

A series of five solutions of constant concentrations of $\mathrm{B}\left(\mathrm{C}_{6} \mathrm{~F}_{5}\right)_{3}$ and of increasing concentrations of 9phosphatriptycene 1 were prepared in $\mathrm{CH}_{2} \mathrm{Cl}_{2}$ under argon according to Table S2. The initial concentration of $\mathrm{B}\left(\mathrm{C}_{6} \mathrm{~F}_{5}\right)_{3}$ was $6.10 \times 10^{-5} \mathrm{M}$. The equilibrium was reached instantaneously and the UV-vis spectra were identical after immediate recording or after $15 \mathrm{~min}$ as indicated by the stable absorbance at the $\lambda^{\max }$ of $\mathrm{B}\left(\mathrm{C}_{6} \mathrm{~F}_{5}\right)_{3}(303 \mathrm{~nm})$ as shown in Figure $\mathrm{S} 1$. The extinction coefficient $\varepsilon$ for $\mathrm{B}\left(\mathrm{C}_{6} \mathrm{~F}_{5}\right)_{3}$ at $\lambda^{\max }=303 \mathrm{~nm}$ was measured twice in $\mathrm{CH}_{2} \mathrm{Cl}_{2}$ and found to be equal to $2.10( \pm 0.07) \times 10^{4} \mathrm{~L} \mathrm{~mol}^{-1} \mathrm{~cm}^{-1}$.

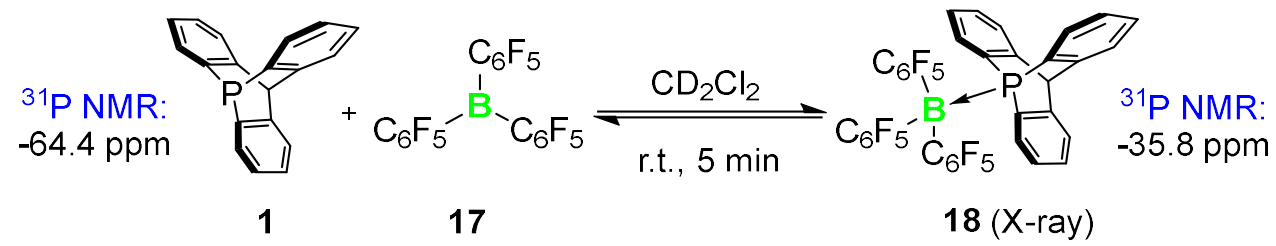


Table S2: Solutions prepared for performing the UV-vis binding titration between $\mathbf{1}$ and $\mathbf{1 7 .}$

\begin{tabular}{cccccc}
\hline$\left[\mathrm{B}\left(\mathrm{C}_{6} \mathrm{~F}_{5}\right)_{3}\right]_{0} / \mathrm{mol}^{-\mathrm{L}^{-1}}$ & $\mathrm{~A}_{\mathrm{eq}}$ & \multicolumn{4}{c}{$\mathrm{A}_{0}-\mathrm{A}_{\mathrm{eq}}\left(\mathrm{A}_{0}-\mathrm{A}_{\mathrm{eq}}\right) / \mathrm{A}_{\mathrm{eq}}[\mathbf{1}]_{0} / \mathrm{mol}^{-\mathrm{L}^{-1}}[\mathbf{1}]_{0}-\left(\left(\mathrm{A}_{0}-\mathrm{A}_{\mathrm{eq}}\right) / \varepsilon_{\mathrm{B}(\mathrm{C} 6 \mathrm{~F} 5) 3}\right.$} \\
\hline $6.10 \times 10^{-5}$ & $1.123\left(=\mathrm{A}_{0}\right)$ & - & - & - & - \\
$6.10 \times 10^{-5}$ & 0.682 & 0.441 & 0.647 & $3.02 \times 10^{-5}$ & $9.20 \times 10^{-6}$ \\
$6.10 \times 10^{-5}$ & 0.510 & 0.613 & 1.202 & $6.03 \times 10^{-5}$ & $3.11 \times 10^{-6}$ \\
$6.10 \times 10^{-5}$ & 0.352 & 0.772 & 2.195 & $9.05 \times 10^{-5}$ & $5.38 \times 10^{-6}$ \\
$6.10 \times 10^{-5}$ & 0.269 & 0.854 & 3.169 & $1.21 \times 10^{-4}$ & $8.03 \times 10^{-5}$ \\
\hline
\end{tabular}

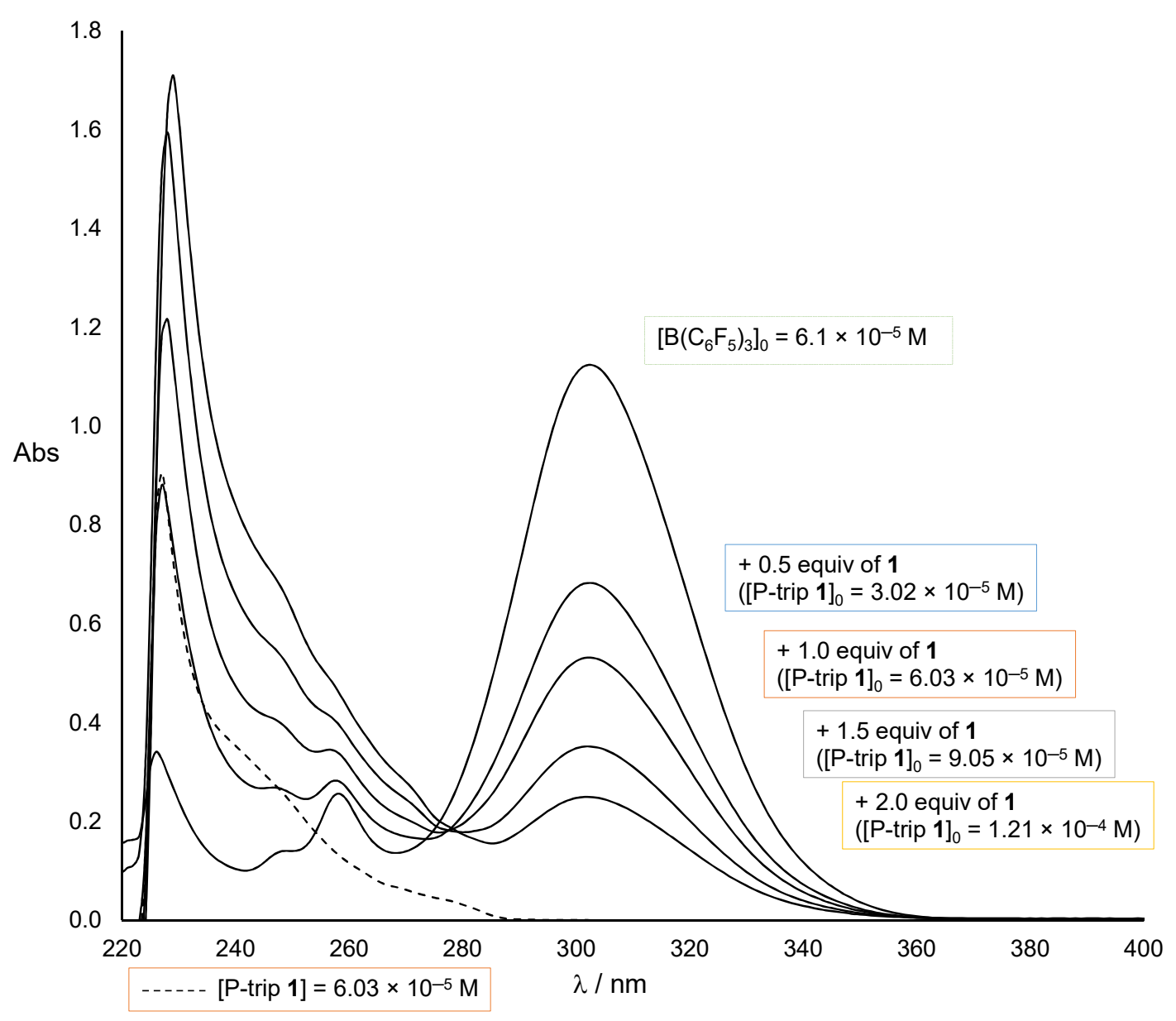

Figure S1. UV-vis measurement of the association constant between 9-phosphatriptycene (1) and $\mathrm{B}_{(}\left(\mathrm{C}_{6} \mathrm{~F}_{5}\right)_{3}$ in $\mathrm{CH}_{2} \mathrm{Cl}_{2}$ at $20^{\circ} \mathrm{C}$.

Because of the proportionality between the absorbance and the concentration of $\mathrm{B}\left(\mathrm{C}_{6} \mathrm{~F}_{5}\right)_{3}(\mathrm{Beer}-\mathrm{Lambert}$ law), the equilibrium constants $K_{\text {eq }}$ can be derived from the initial absorbance $\mathrm{A}_{0}$ of $\mathrm{B}\left(\mathrm{C}_{6} \mathrm{~F}_{5}\right)_{3}$ and its absorbance at equilibrium $\mathrm{A}_{\mathrm{eq}}$ after adding a defined amount of 9-phosphatriptycene $\mathbf{1}$ and after equilibration of the solutions $(<5 \mathrm{~min})$. 


$$
\begin{gathered}
K_{\text {eq }}=\frac{[\text { Lewis adduct } \mathbf{1 8}]_{\mathrm{eq}}}{\left[\mathrm{B}\left(\mathrm{C}_{6} \mathrm{~F}_{5}\right)_{3} \mathbf{1 7}\right]_{\mathrm{eq}}[\mathbf{1}]_{\mathrm{eq}}}=\frac{A_{0}-A_{\mathrm{eq}}}{A_{\mathrm{eq}} \times[\mathbf{1}]_{\mathrm{eq}}} \\
{[\mathbf{1}]_{\mathrm{eq}}=[\mathbf{1}]_{0}-\frac{A_{0}-A_{\mathrm{eq}}}{\varepsilon}}
\end{gathered}
$$

The plot of $\left(\mathrm{A}_{0}-\mathrm{A}_{\mathrm{eq}}\right) / \mathrm{A}_{\mathrm{eq}}$ versus the concentration of $[\mathbf{1}]_{\mathrm{eq}}$ is linear (Figure $\mathrm{S} 2$ ), with a slope equal to $K_{\mathrm{eq}}$ (Figure S2).

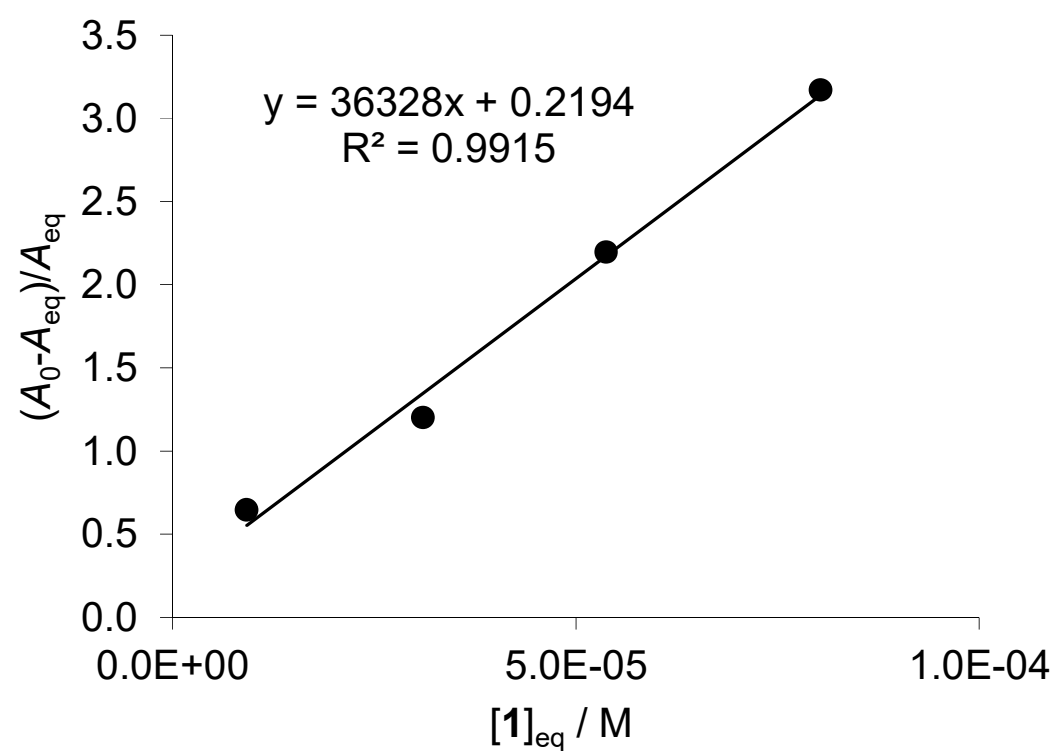

Figure S2. Determination of the association constant between 9-phosphatriptycene (1) and $\mathrm{B}\left(\mathrm{C}_{6} \mathrm{~F}_{5}\right)_{3}$ in $\mathrm{CH}_{2} \mathrm{Cl}_{2}$ at $20^{\circ} \mathrm{C}$. 


\section{Copies of all NMR and HRMS spectra}

2.1 9-phospha-10-hydroxytriptycene $8\left({ }^{1} \mathrm{H},{ }^{31} \mathrm{P},{ }^{13} \mathrm{C}\right.$-NMR, HRMS $)$
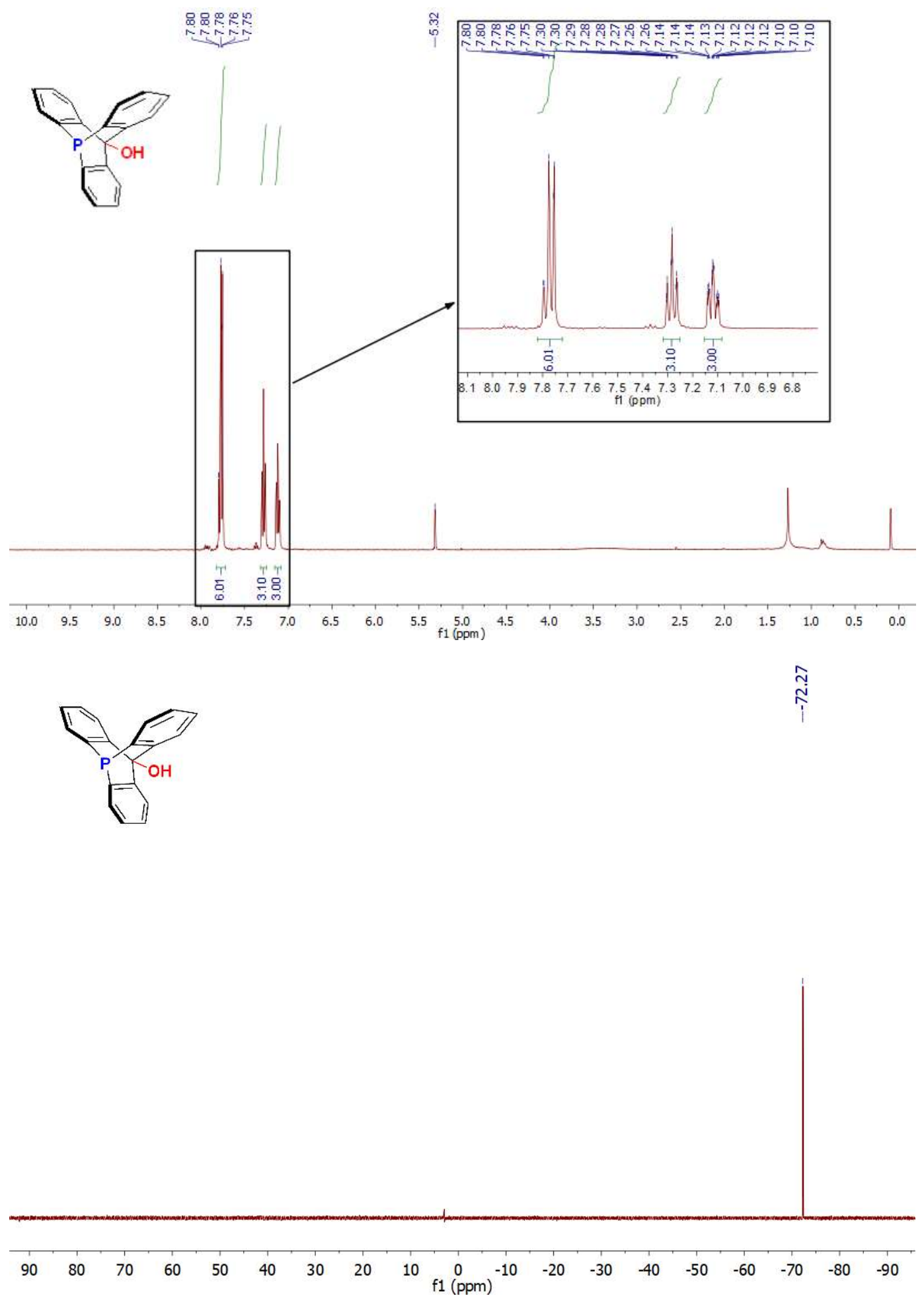

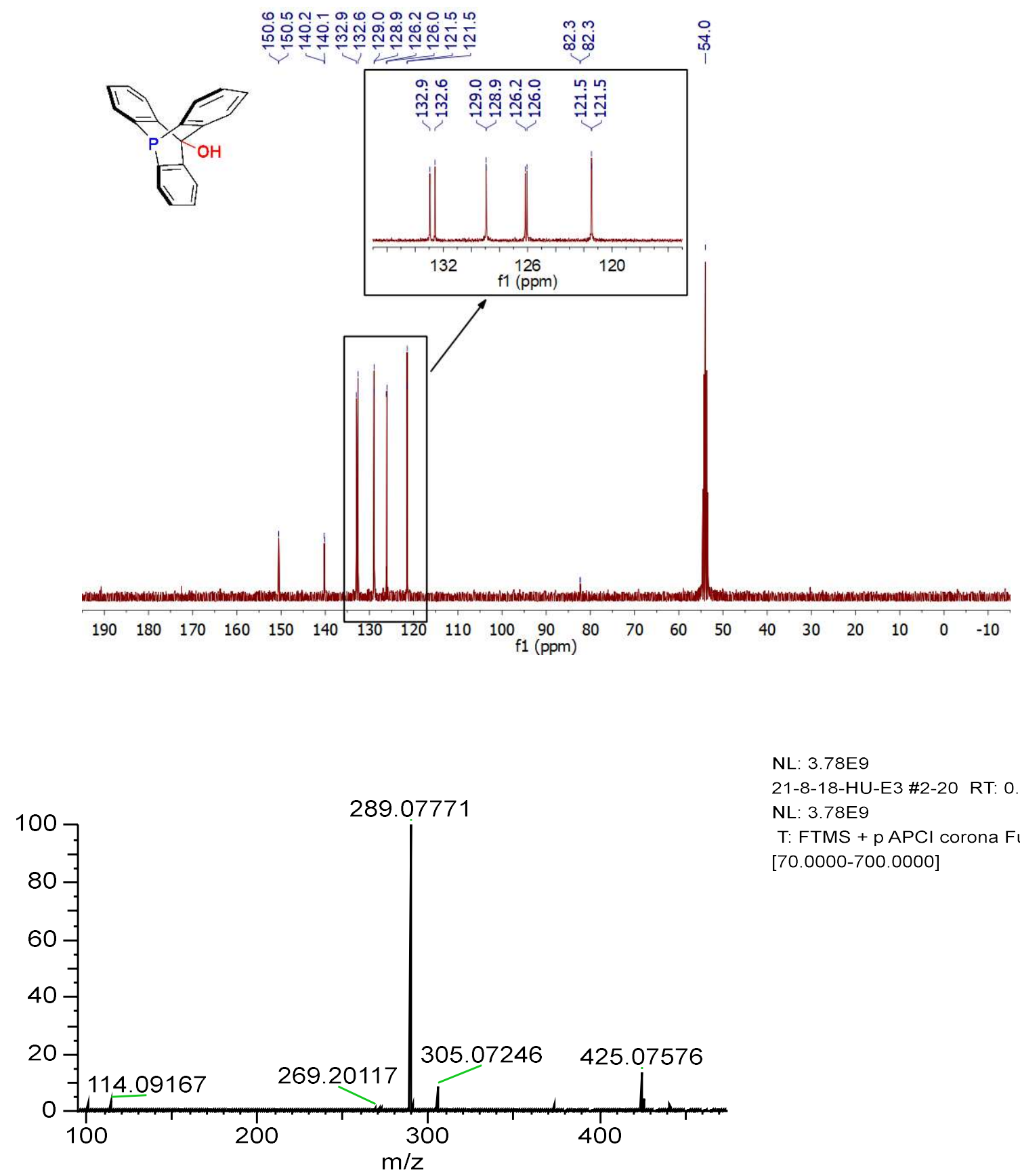

$\mathrm{NL}: 3.78 \mathrm{E} 9$

21-8-18-HU-E3 \#2-20 RT: 0.01-0.09 AV: 19 $\mathrm{NL}: 3.78 \mathrm{E} 9$

T: FTMS + p APCl corona Full ms [70.0000-700.0000] 
2.2 9-phospatriptycene-10-phenyl thiocarbonate $9\left({ }^{1} \mathrm{H},{ }^{31} \mathrm{P},{ }^{13} \mathrm{C}\right.$-NMR, HRMS $)$
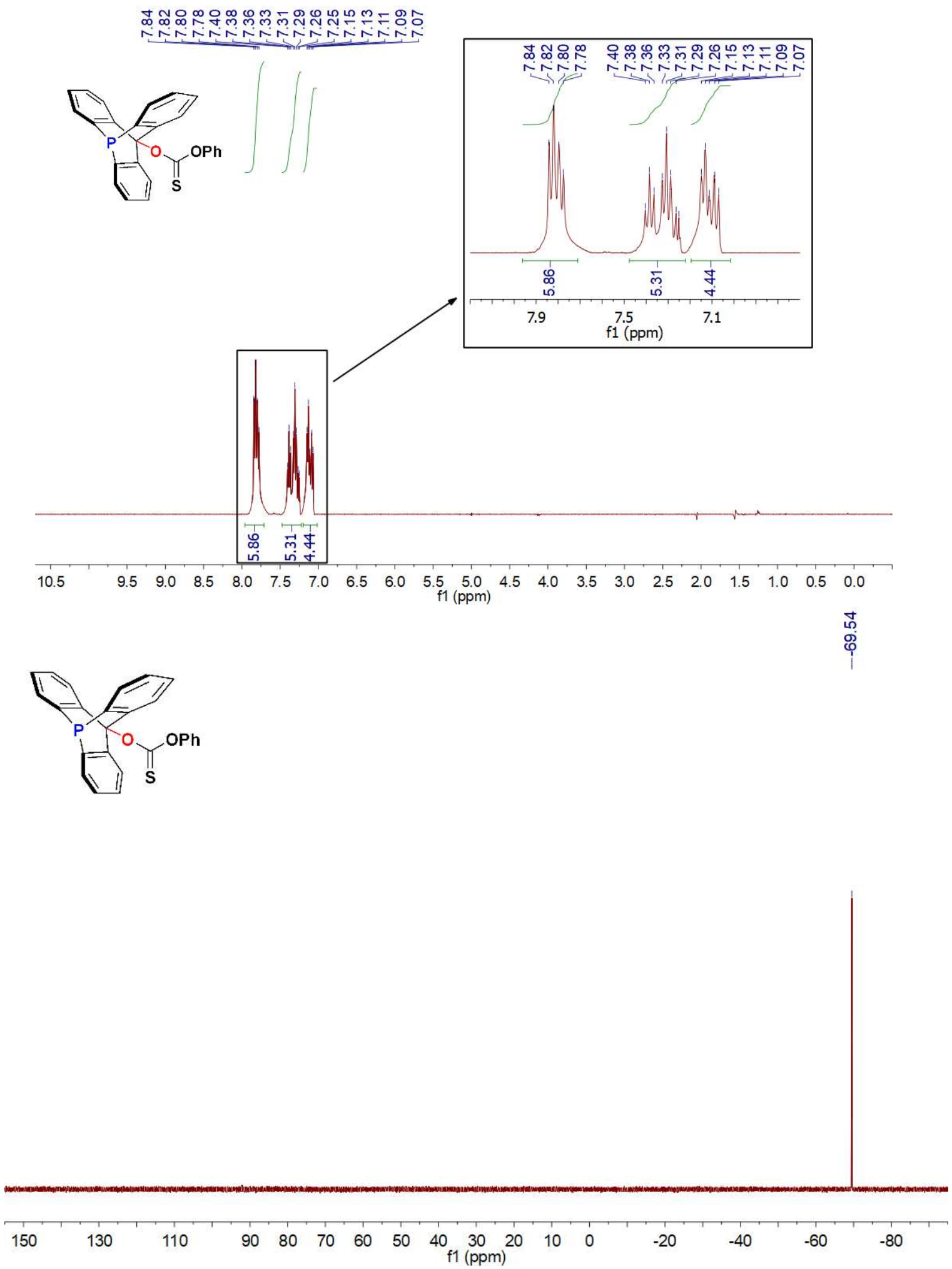


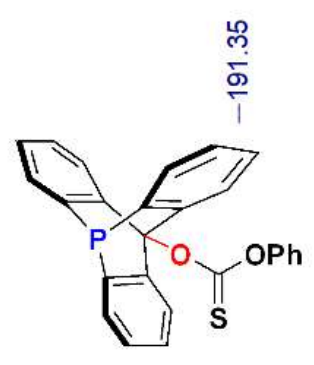

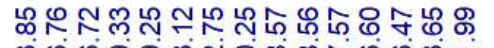

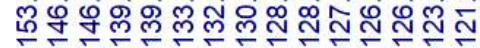

ले

मुं

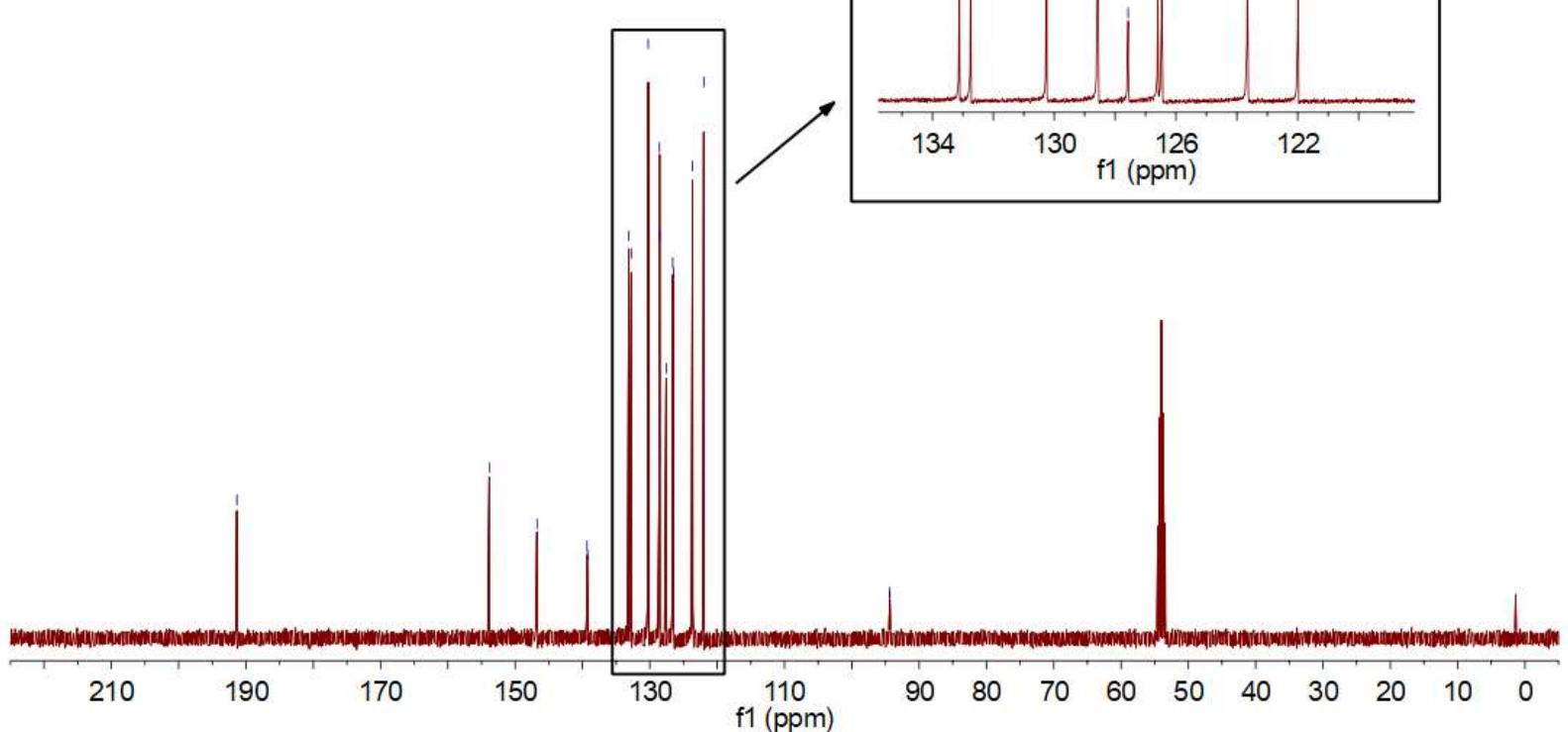

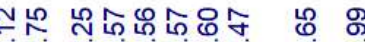

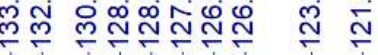

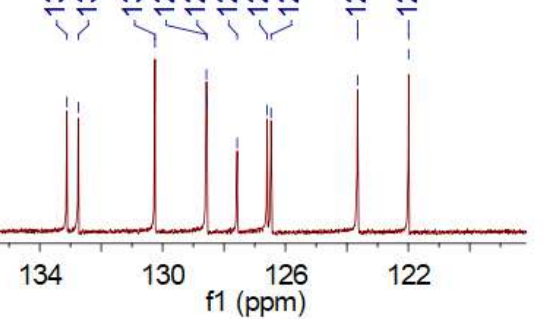


2.3 9-phospatriptycene $\mathbf{1}\left({ }^{1} \mathrm{H},{ }^{31} \mathrm{P},{ }^{13} \mathrm{C}\right.$-NMR, HRMS $)$
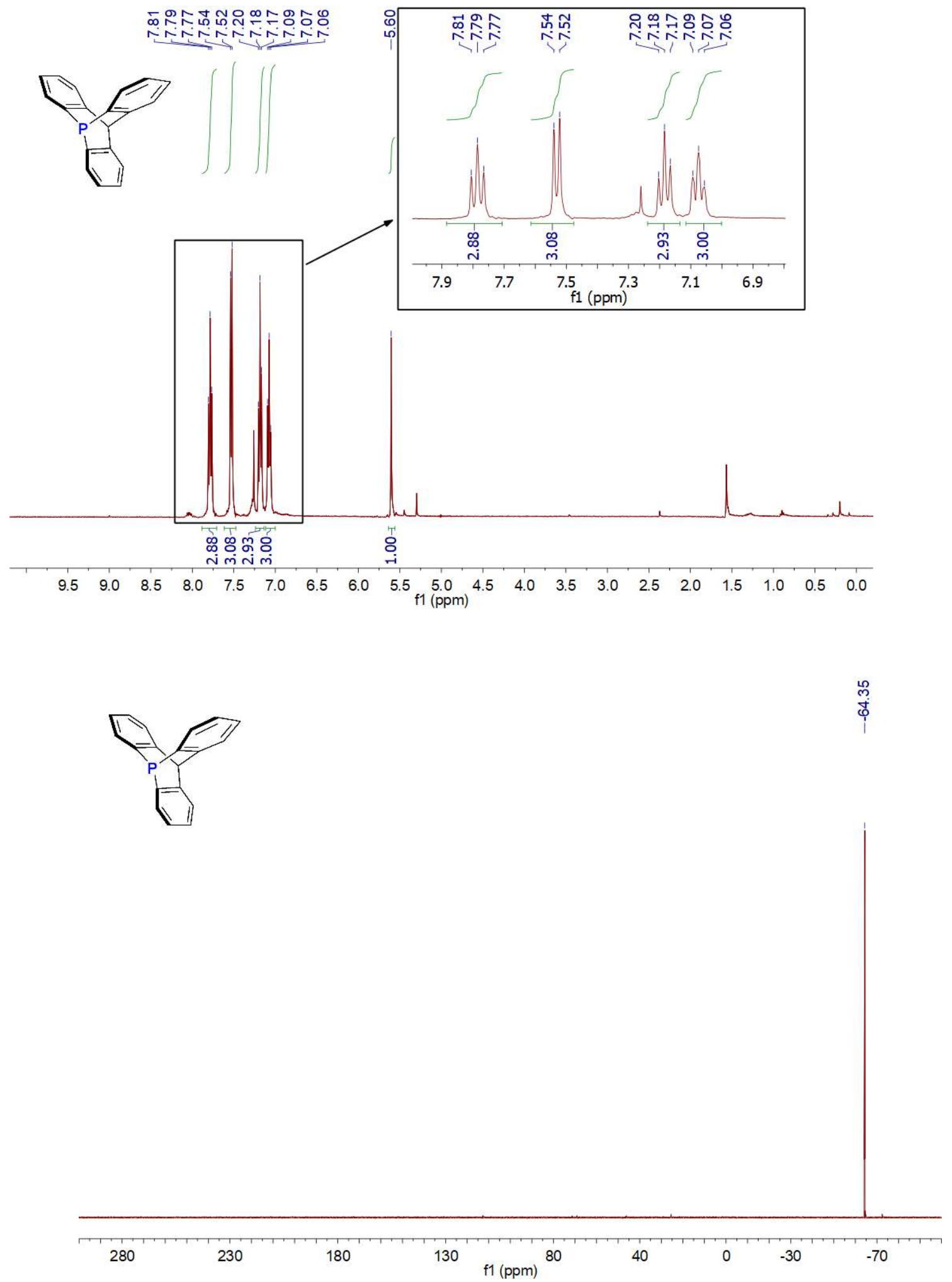

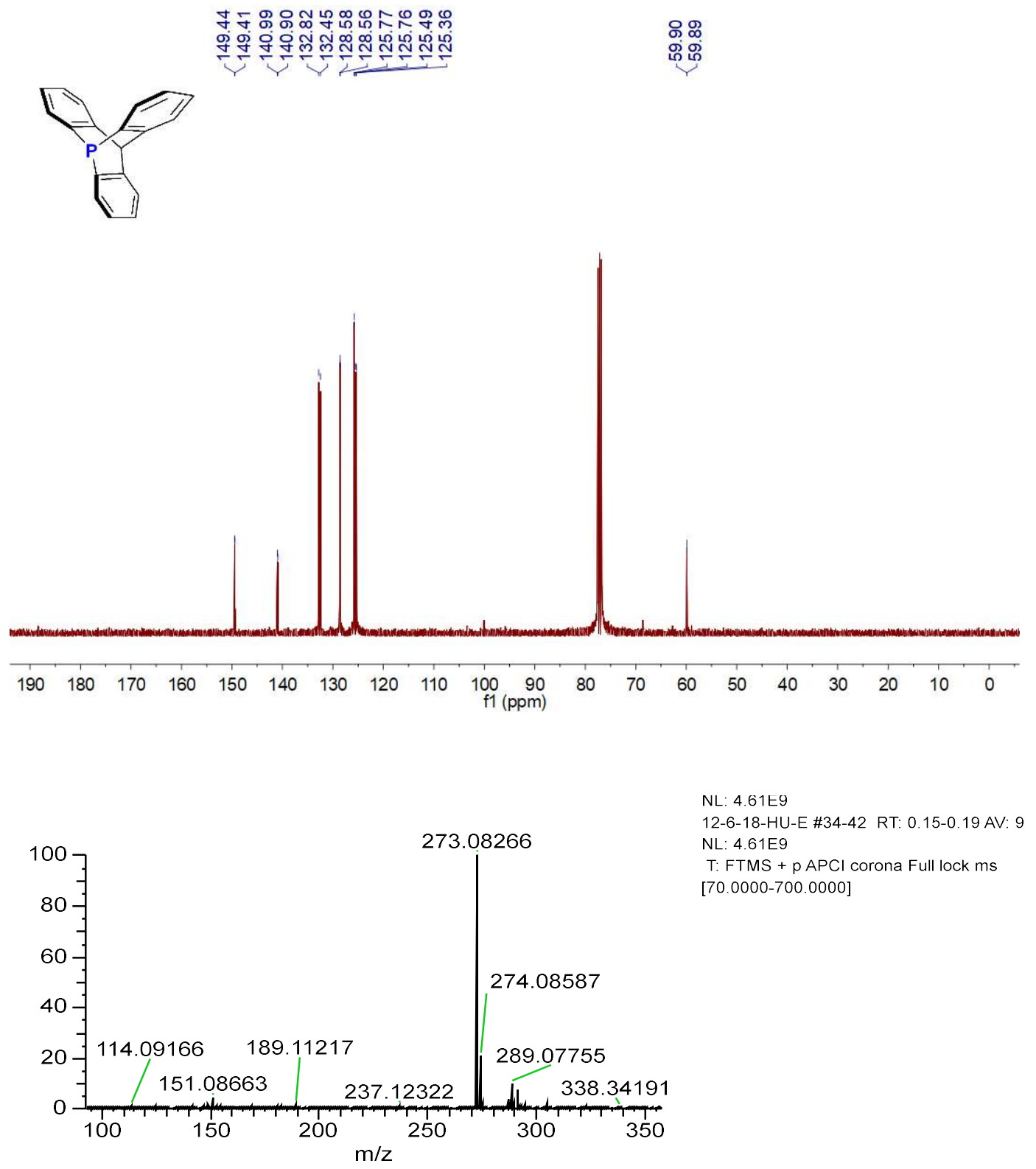

$\mathrm{NL}: 4.61 \mathrm{E} 9$

12-6-18-HU-E \#34-42 RT: 0.15-0.19 AV: 9

$\mathrm{NL}: 4.61 \mathrm{E} 9$

T: FTMS + p APCI corona Full lock ms

[70.0000-700.0000] 
2.4 9-phospatriptycene- benzhydryl tetrafluoroborate complex $\mathbf{1 3 b}\left({ }^{1} \mathrm{H},{ }^{31} \mathrm{P},{ }^{11} \mathrm{~B},{ }^{19} \mathrm{~F}-\mathrm{NMR}\right)$
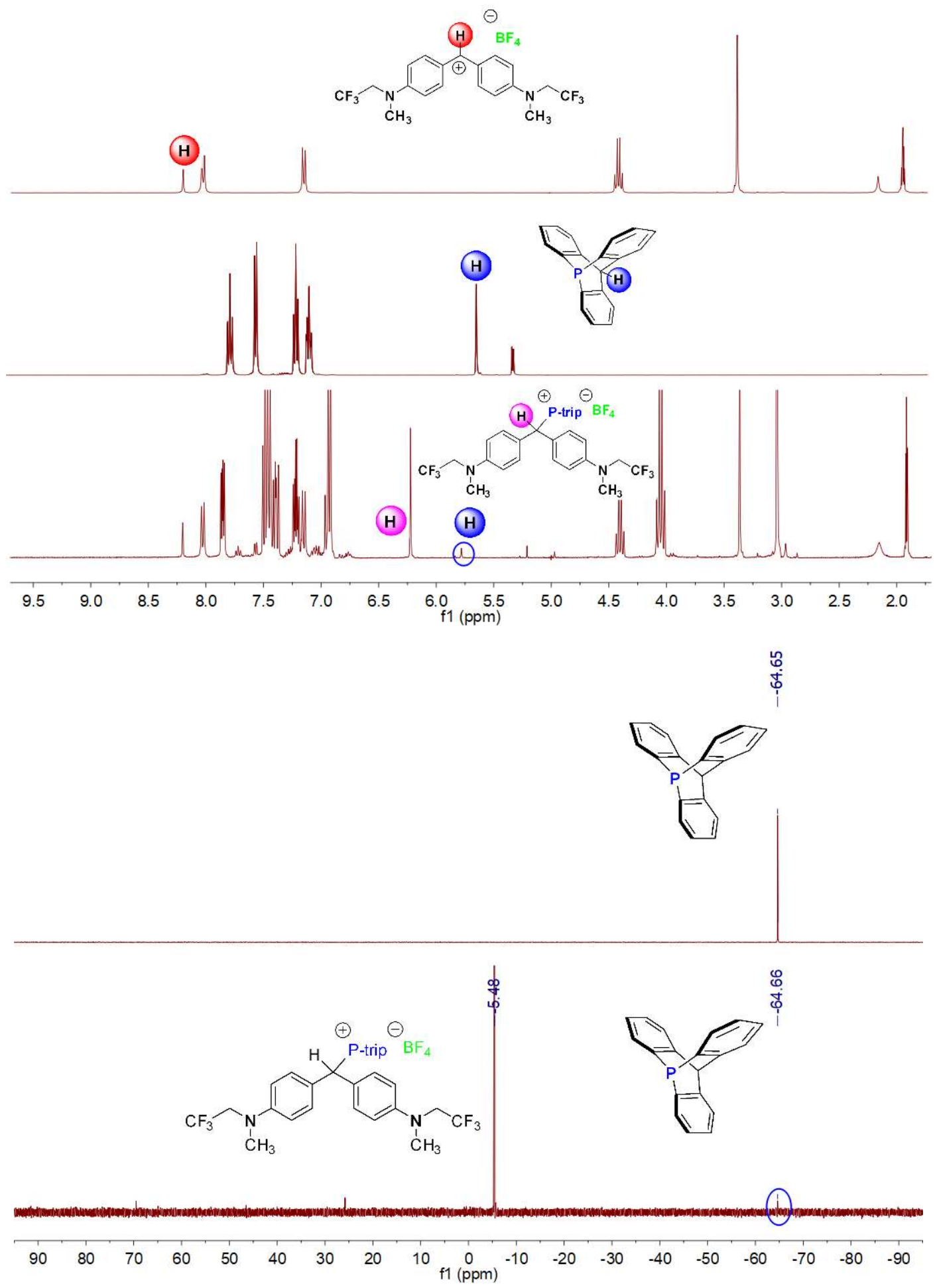

S12 
ํำ

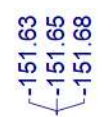
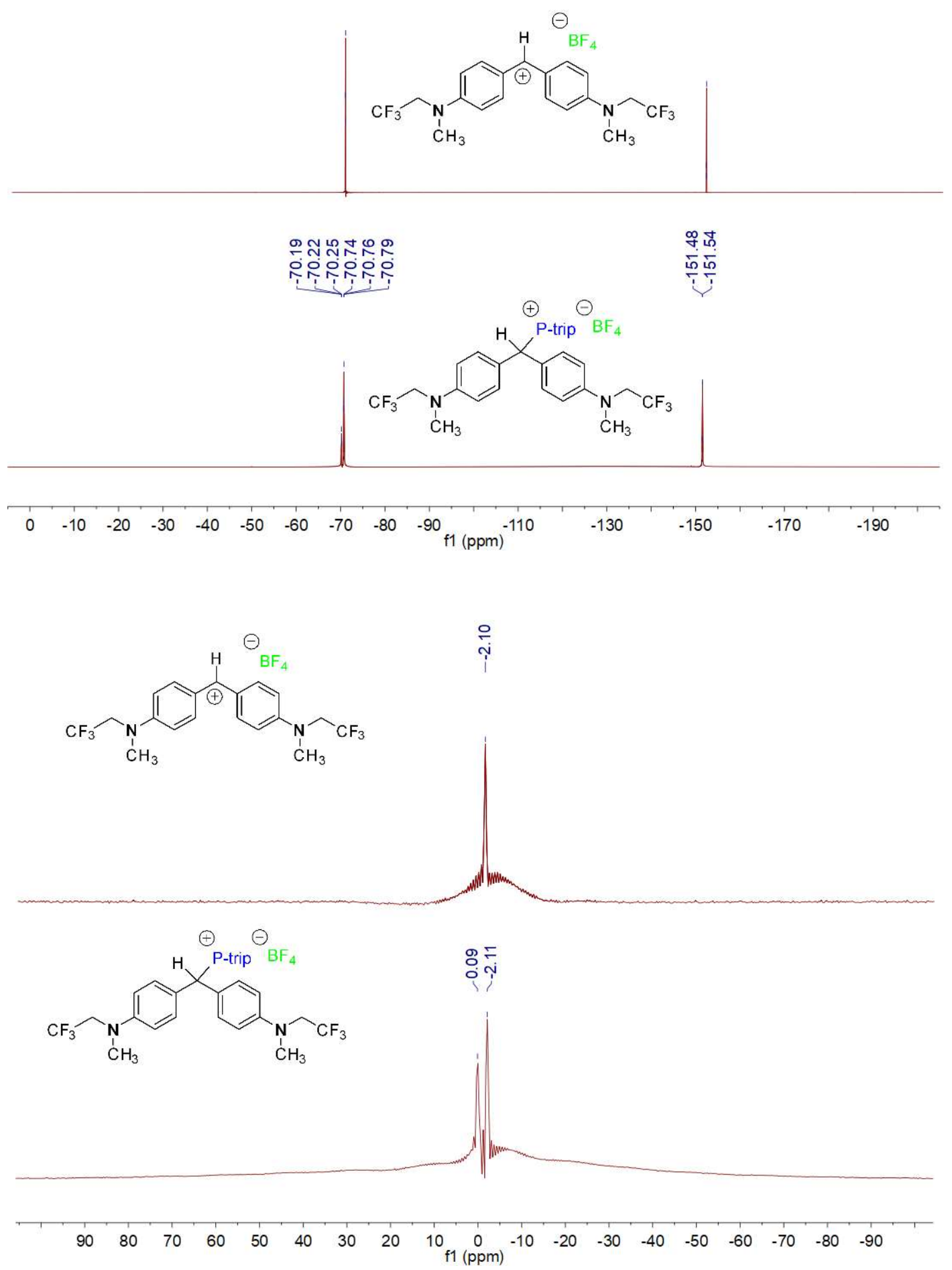

S13 
2.5 9-phospatriptycene-tritylium tetrafluoroborate complex with 9-phospatriptycene and tritylium tetrafluoroborate $\mathbf{1 6}\left({ }^{1} \mathrm{H},{ }^{31} \mathrm{P},{ }^{13} \mathrm{C}-\mathrm{NMR}, \mathrm{HRMS}\right)$

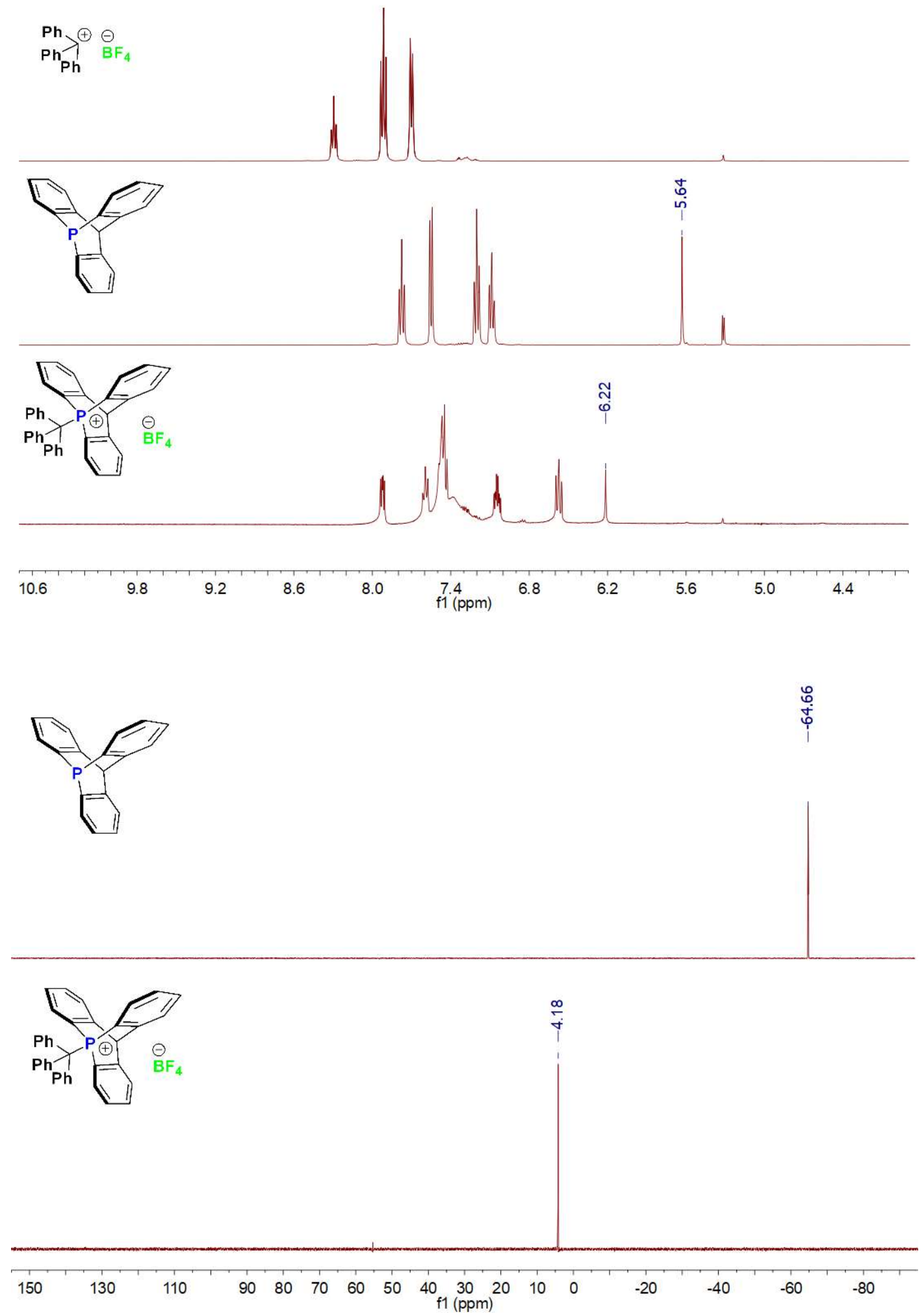




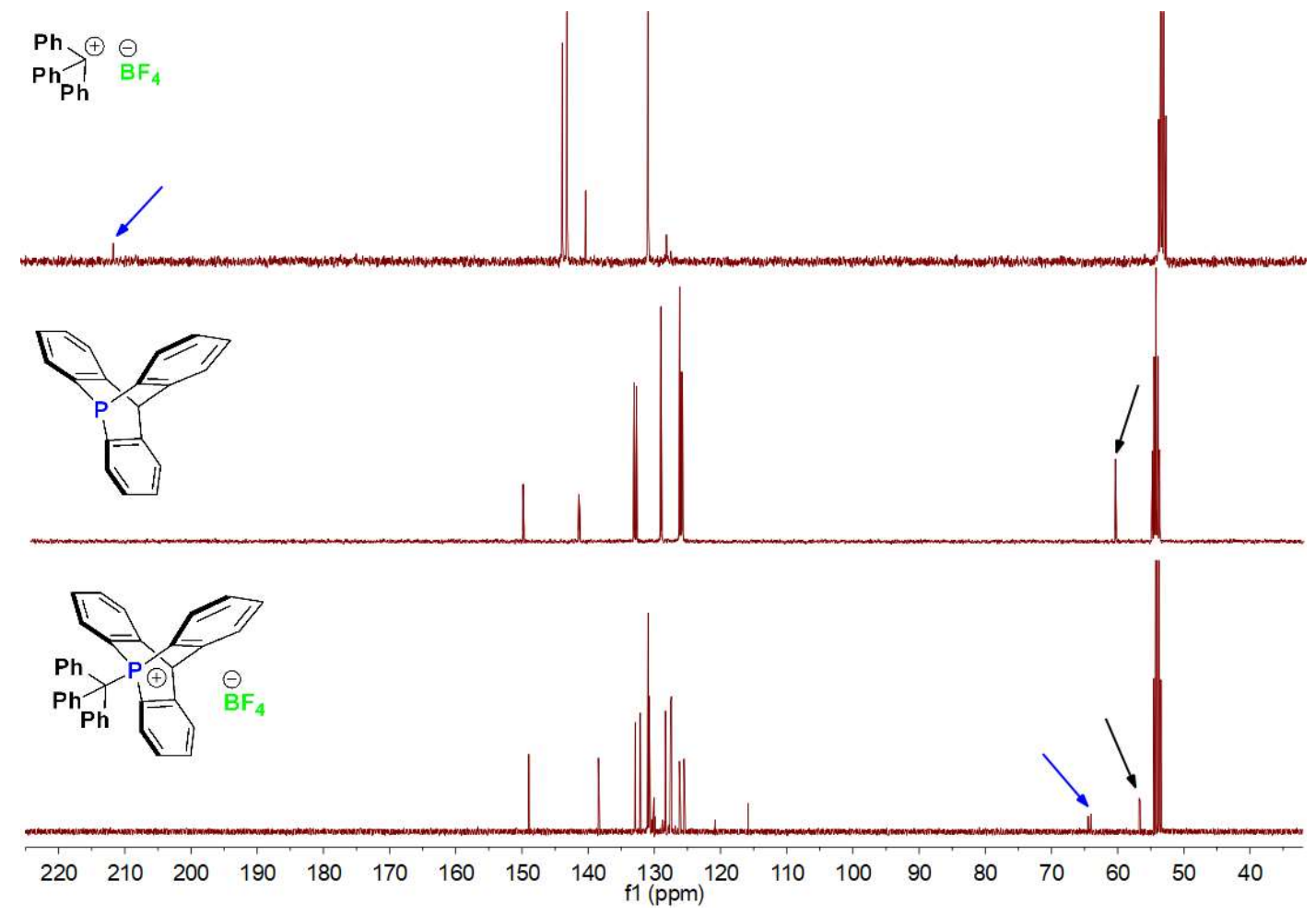

NL: $8.42 \mathrm{E} 8$

11-4-19-HU-E4 \#18-47 RT: 0.08-0.21 AV: 26 NL: $8.42 \mathrm{E} 8$

T: FTMS + p ESI Full lock ms

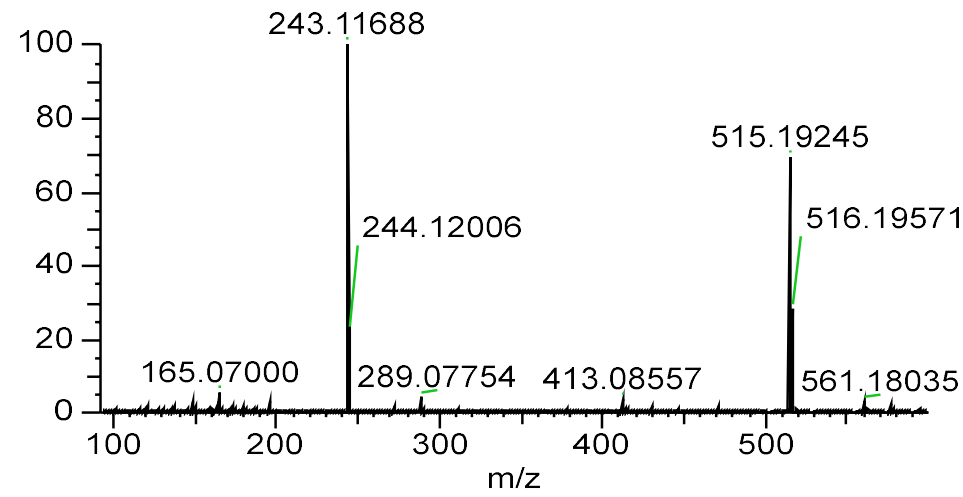

[70.0000-700.0000] 
2.69-phospatriptycene-tris(pentafluorophenyl)borane complex 18 compared with 9-phospatriptycene $\mathbf{1}\left({ }^{1} \mathrm{H},{ }^{31} \mathrm{P}-\mathrm{NMR}\right)$
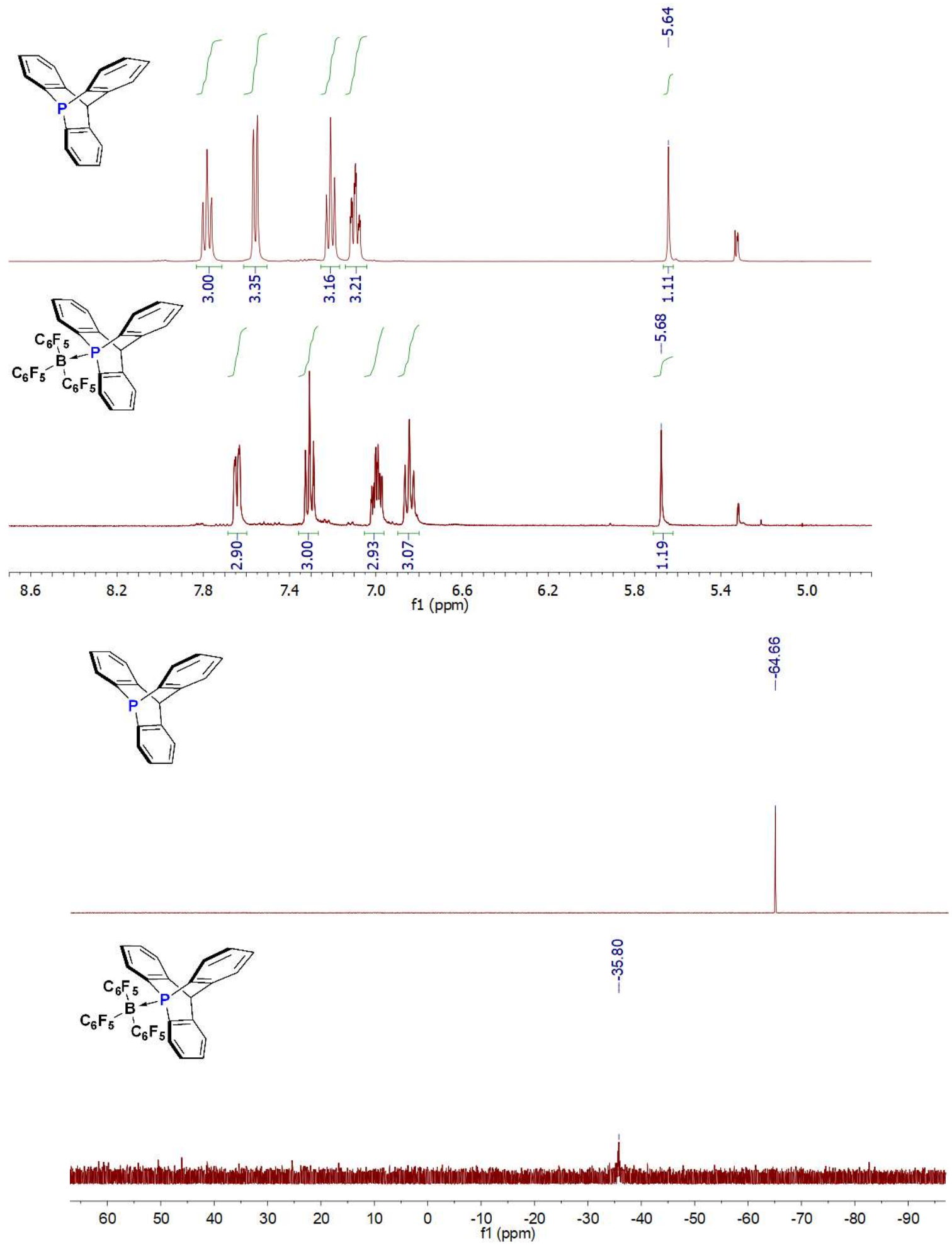


\section{Single-crystal $X$-ray diffraction analysis}

Details of single-crystal X-ray diffraction data collection and determination are given in Table S3. Molecular structure of the compound $\mathbf{8}$ is shown in Figure S3.

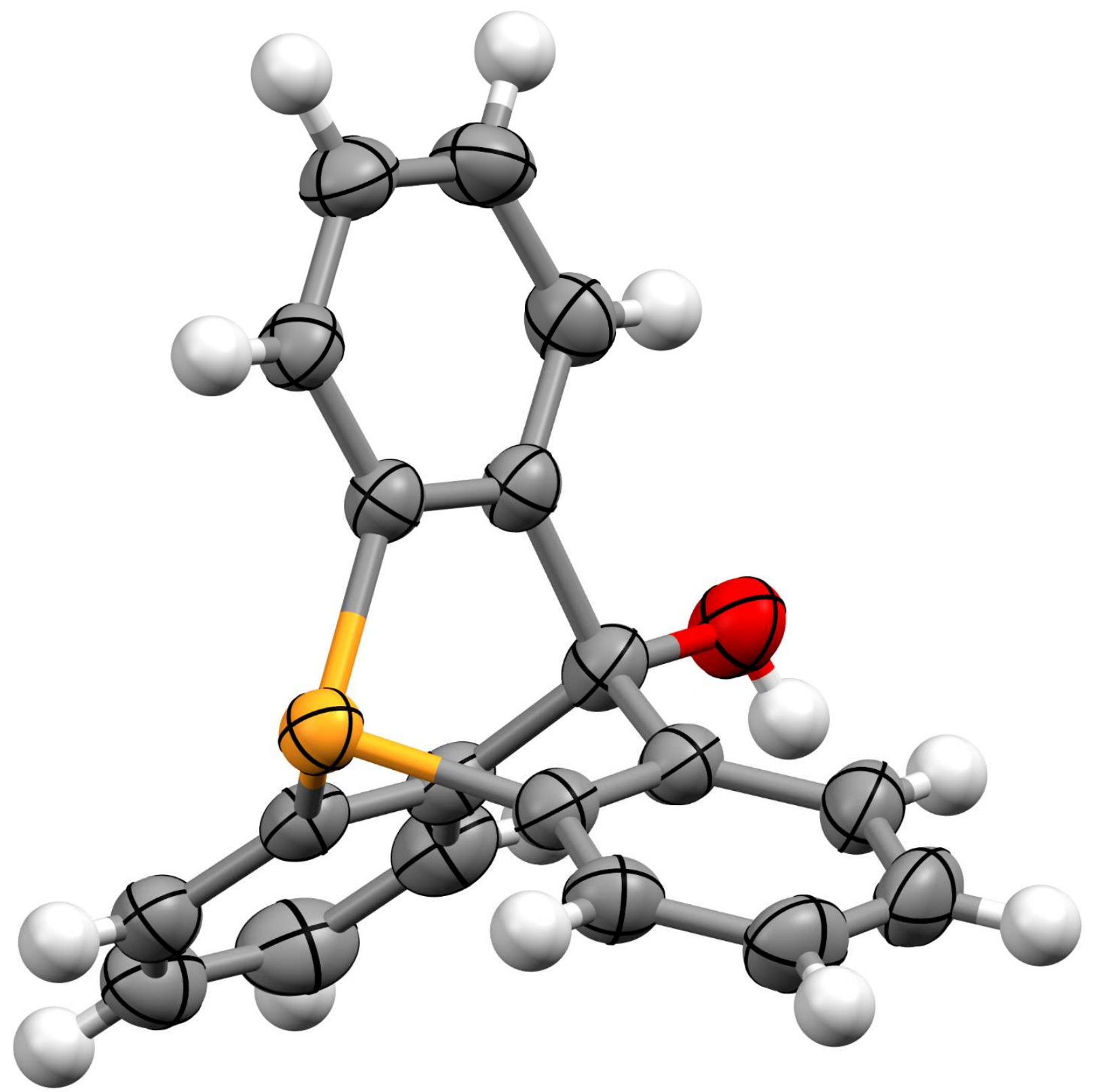

Figure S3. Molecular structure of the compound 8, shown with thermal ellipsoids of 50\% probability representation. 


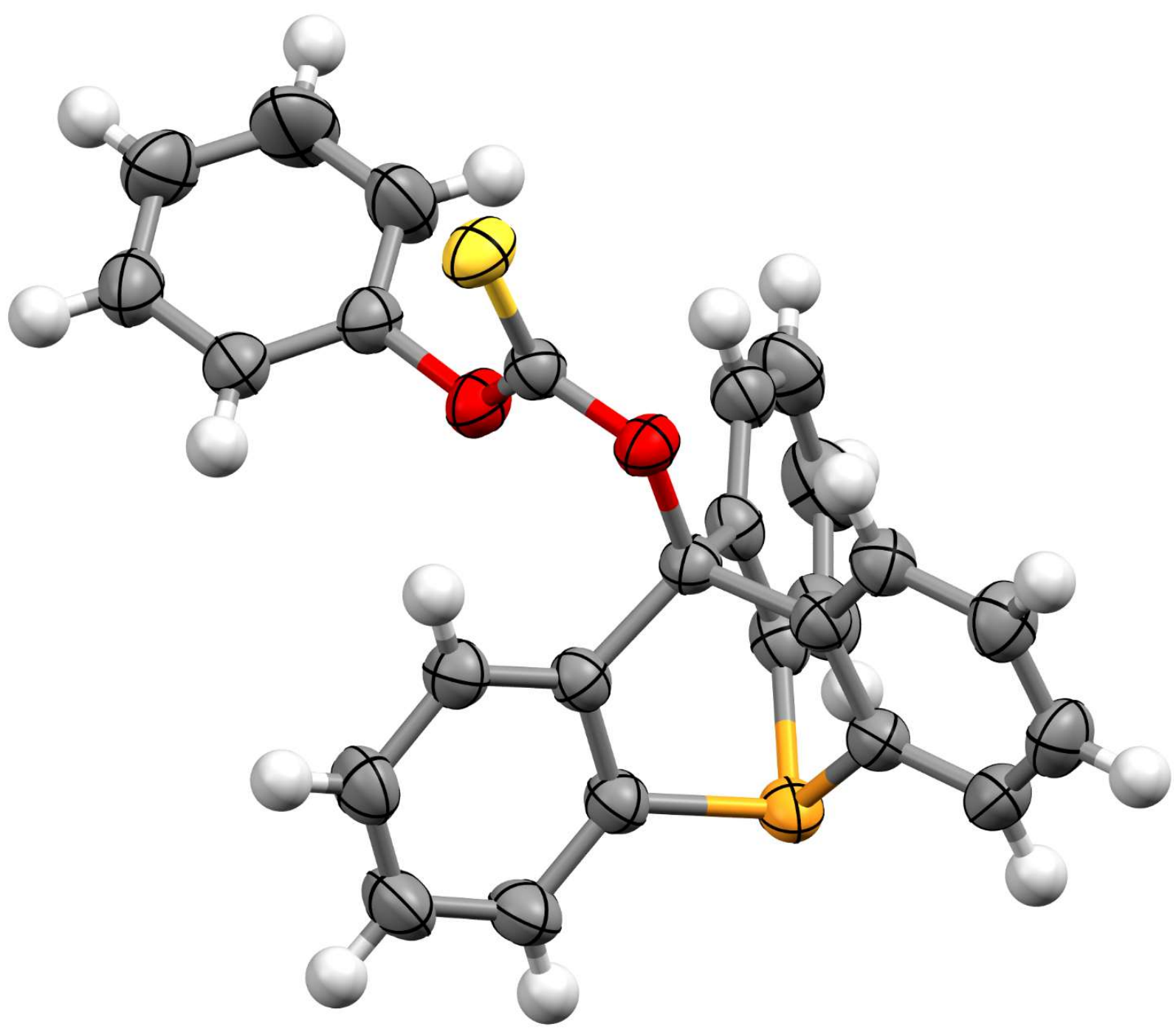

Figure S4. Molecular structure of the compound 9, shown with thermal ellipsoids of 50\% probability representation. Only one out of two molecules in asymmetric unit is shown for clarity. 


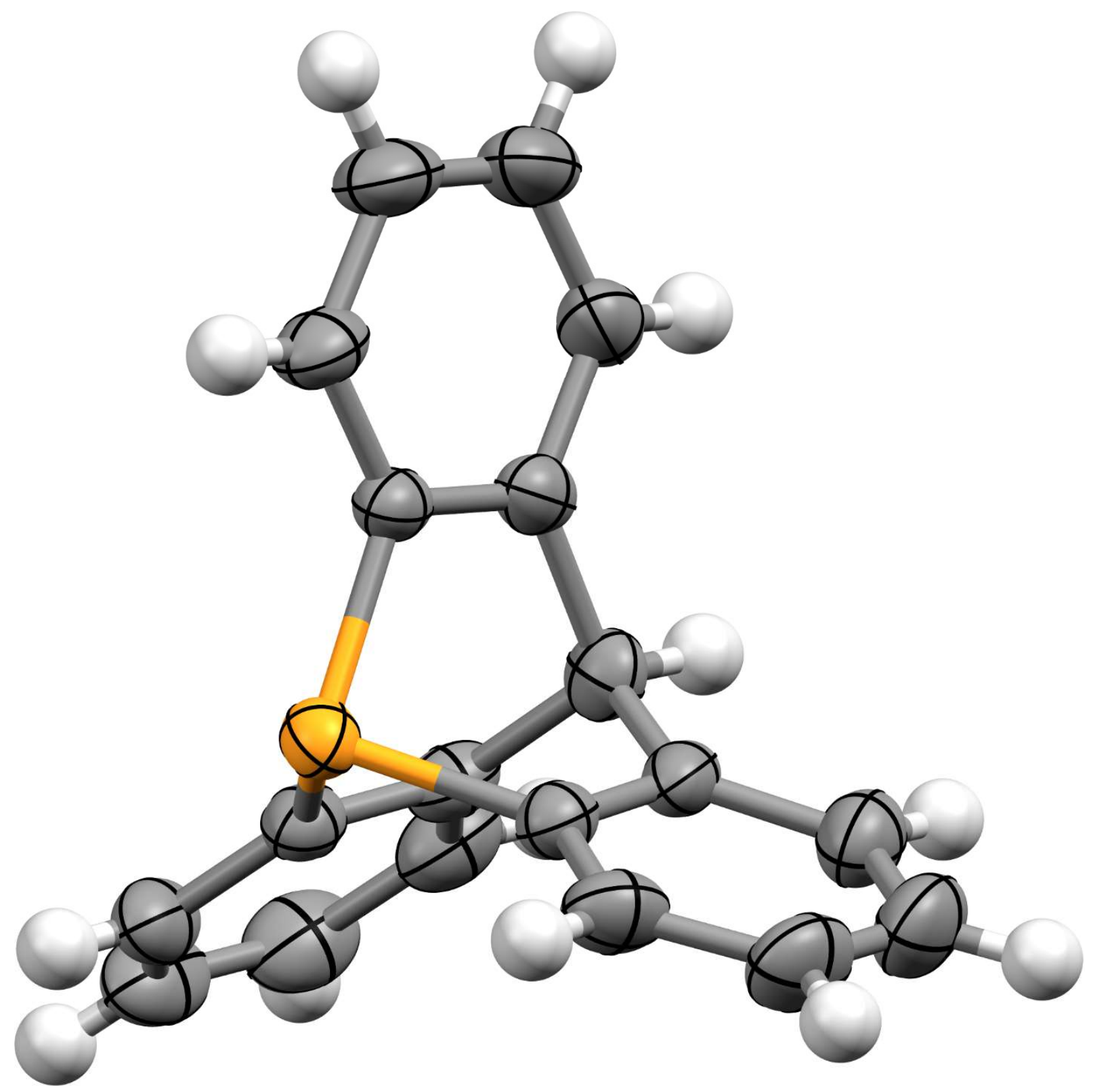

Figure S5. Molecular structure of the compound 1 at $295 \mathrm{~K}$, shown with thermal ellipsoids of $50 \%$ probability representation. Minor component of disorder is omitted for clarity. 


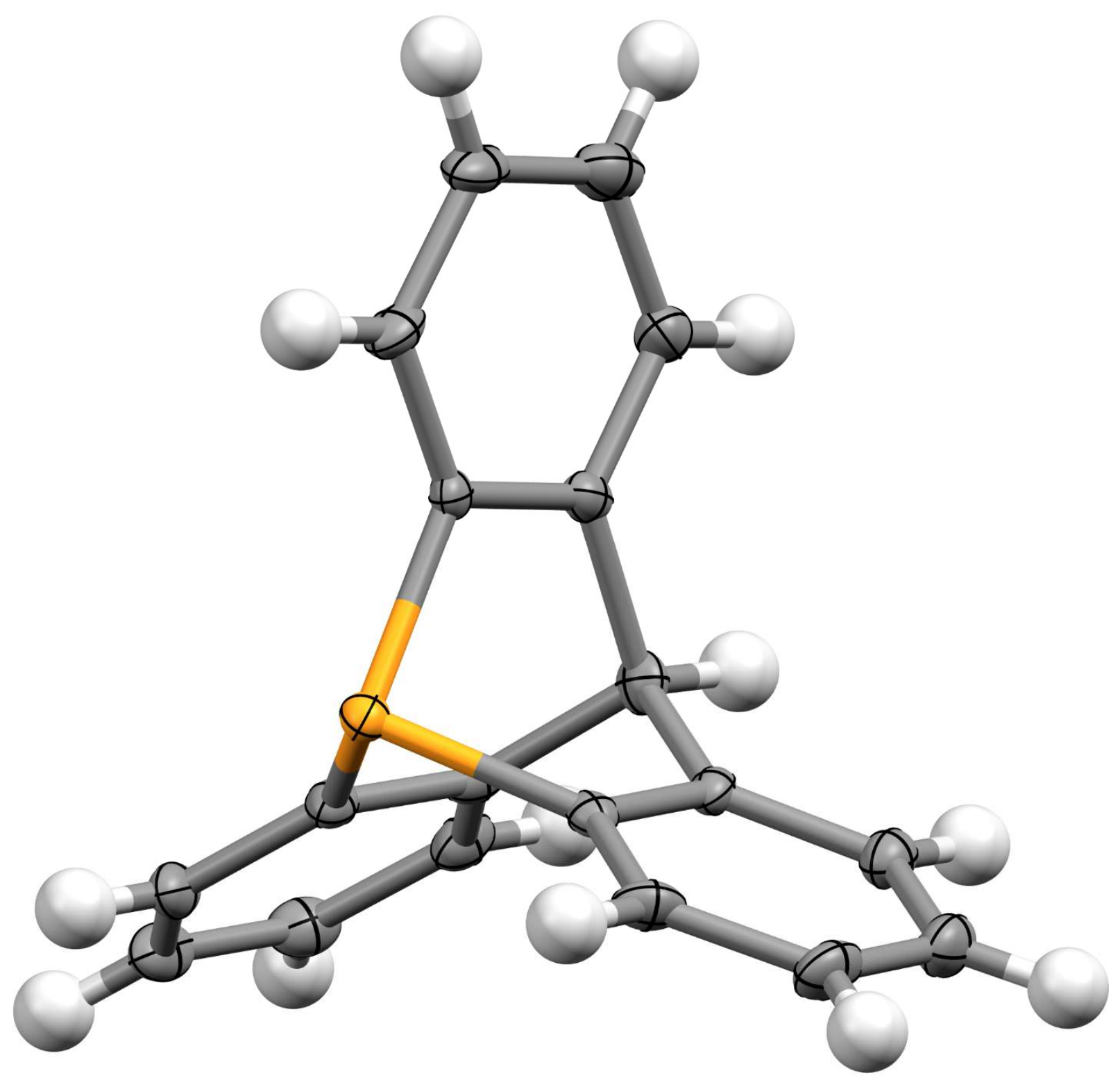

Figure S6. Molecular structure of the compound 1 at $100 \mathrm{~K}$, shown with thermal ellipsoids of $50 \%$ probability representation. Minor component of disorder is omitted for clarity. 


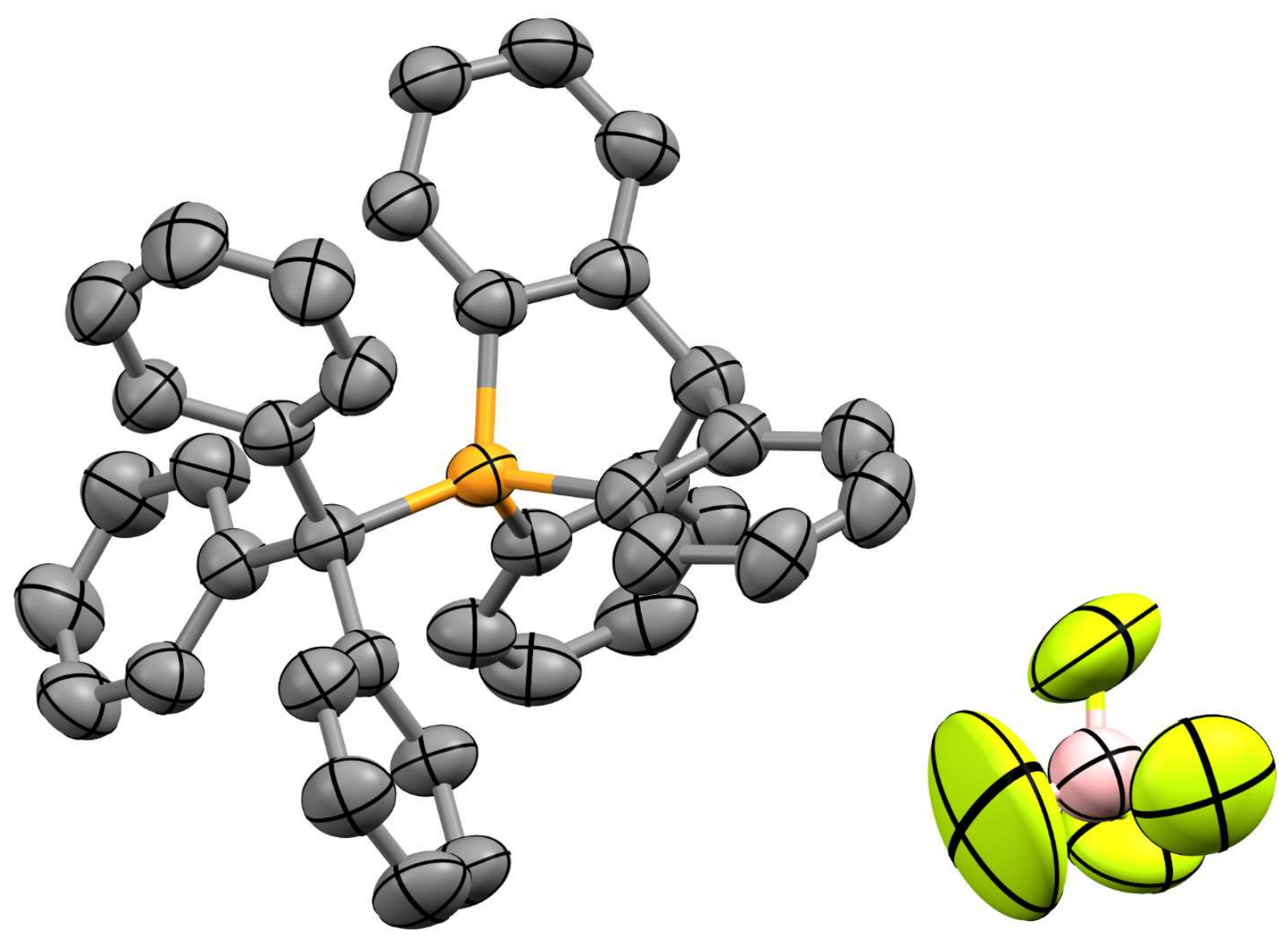

Figure S7. Molecular structure of the compound 16, shown with thermal ellipsoids of 50\% probability representation. Hydrogen atoms are omitted for clarity. 


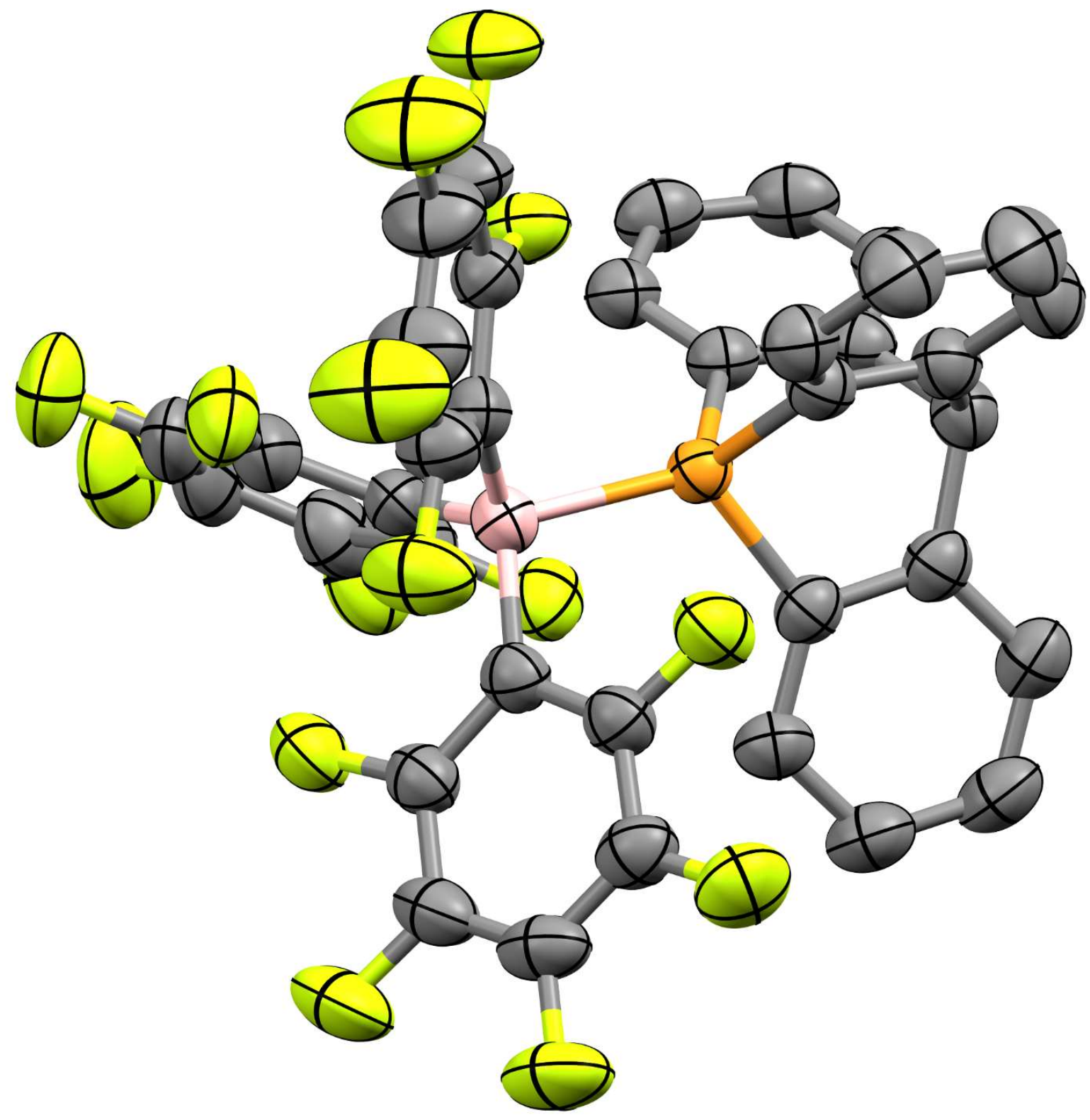

Figure S8. Molecular structure of the compound 18, shown with thermal ellipsoids of 50\% probability representation. Hydrogen atoms and disordered dichloromethane molecule are omitted for clarity. 
Table S3: Crystal data for compounds 1, 8, 9, 16 and 18.

\begin{tabular}{|c|c|c|c|c|}
\hline & \|\| & \|\|$^{\mathrm{S}}$ & $(11)$ & $\mathbf{1}, 100 \mathrm{~K}$ \\
\hline Chemical formula & $\mathrm{C}_{19} \mathrm{H}_{13} \mathrm{OP}$ & $\mathrm{C}_{26} \mathrm{H}_{17} \mathrm{O}_{2} \mathrm{PS}$ & $\mathrm{C}_{19} \mathrm{H}_{13} \mathrm{P}$ & $\mathrm{C}_{19} \mathrm{H}_{13} \mathrm{P}$ \\
\hline$M_{\mathrm{r}}$ & 288.26 & 424.42 & 272.26 & 272.26 \\
\hline $\begin{array}{l}\text { Crystal system, space } \\
\text { group }\end{array}$ & Triclinic, $P^{-} 1$ & Monoclinic, $P 2_{1} / c$ & Monoclinic, $C c$ & Monoclinic, $C c$ \\
\hline Temperature (K) & \multicolumn{3}{|c|}{295} & 100 \\
\hline$a, b, c(\AA)$ & $\begin{array}{l}7.2966(4), 8.2064(4), \\
13.0501(7)\end{array}$ & $\begin{array}{l}25.0052(2), \\
8.40856(8), \\
20.01476(18)\end{array}$ & $\begin{array}{l}15.0310(5), \\
8.2009(2), \\
13.1609(4)\end{array}$ & $\begin{array}{l}14.8144(10), 8.1417(3), \\
13.0081(9)\end{array}$ \\
\hline$\alpha, \beta, \gamma\left(^{\circ}\right)$ & $\begin{array}{l}82.694(4), 80.962(4), \\
69.948(5)\end{array}$ & 90, 94.2309(8), 90 & $90,120.422(4), 90$ & $90,120.325(10), 90$ \\
\hline$V\left(\AA^{3}\right)$ & $722.67(7)$ & 4196.79(7) & 1398.95(9) & 1354.29(19) \\
\hline$Z$ & 2 & 8 & 4 & 4 \\
\hline Radiation type & \multicolumn{2}{|c|}{$\mathrm{Cu} K \alpha$} & \multicolumn{2}{|c|}{ Mo $K \alpha$} \\
\hline$\mu\left(\mathrm{mm}^{-1}\right)$ & 1.63 & 2.25 & 0.18 & 0.19 \\
\hline Crystal size (mm) & $0.15 \times 0.11 \times 0.03$ & $0.49 \times 0.19 \times 0.12$ & $0.79 \times 0.60 \times 0.10$ & $0.70 \times 0.52 \times 0.18$ \\
\hline Diffractometer & \multicolumn{4}{|c|}{ Oxford Diffraction Xcalibur, Ruby, Gemini Ultra } \\
\hline Absorption correction & \multicolumn{2}{|c|}{ Analytical } & \multicolumn{2}{|c|}{ Gaussian } \\
\hline$T_{\min }, T_{\max }$ & $0.844,0.957$ & $0.541,0.800$ & $0.327,1.000$ & $0.571,1.000$ \\
\hline $\begin{array}{l}\text { No. of measured, } \\
\text { independent and } \\
\text { observed }[I>2 \sigma(I)] \\
\text { reflections }\end{array}$ & $5760,2557,2283$ & $22983,7384,7032$ & $19008,4808,4319$ & $4619,2900,2711$ \\
\hline$R_{\text {int }}$ & 0.021 & 0.019 & 0.025 & 0.023 \\
\hline$(\sin \theta / \lambda)_{\max }\left(\AA^{-1}\right)$ & 0.598 & 0.598 & 0.761 & 0.760 \\
\hline $\begin{array}{l}R\left[F^{2}>2 \sigma\left(F^{2}\right)\right] \\
w R\left(F^{2}\right), S\end{array}$ & $0.037,0.104,1.05$ & $0.037,0.095,1.10$ & $0.028,0.077,1.01$ & $0.034,0.083,1.06$ \\
\hline No. of reflections & 2557 & 7384 & 4808 & 2900 \\
\hline No. of parameters & 193 & 541 & 362 & 362 \\
\hline No. of restraints & 0 & 0 & 818 & 818 \\
\hline $\mathrm{H}$-atom treatment & $\begin{array}{l}\mathrm{H} \text { atoms treated by a } \\
\text { mixture of } \\
\text { independent and } \\
\text { constrained } \\
\text { refinement }\end{array}$ & \multicolumn{3}{|c|}{ H-atom parameters constrained } \\
\hline$\Delta \rho_{\max }, \Delta \rho_{\min }\left(\mathrm{e} \AA^{-3}\right)$ & $0.31,-0.21$ & $0.33,-0.42$ & $0.12,-0.16$ & $0.30,-0.21$ \\
\hline $\begin{array}{l}\text { CCDC deposition } \\
\text { number }\end{array}$ & 1905038 & 1905039 & 1905040 & 1906080 \\
\hline
\end{tabular}




\begin{tabular}{|c|c|c|}
\hline Chemical formula & $\mathrm{C}_{38} \mathrm{H}_{28} \mathrm{P}^{+} \cdot \mathrm{BF}_{4}^{-}$ & $\begin{array}{l}\mathrm{C}_{37} \mathrm{H}_{13} \mathrm{BF}_{15} \mathrm{P} \cdot 0.711\left(\mathrm{CH}_{2}\right. \\
\left.\mathrm{Cl}_{2}\right)\end{array}$ \\
\hline$M_{\mathrm{r}}$ & 602.39 & 844.71 \\
\hline $\begin{array}{l}\text { Crystal system, space } \\
\text { group }\end{array}$ & Trigonal, $R^{-} 3 c: H$ & Monoclinic, $P 2_{1} / c$ \\
\hline Temperature $(\mathrm{K})$ & \multicolumn{2}{|c|}{295} \\
\hline$a, b, c(\AA)$ & $\begin{array}{l}13.4876(2), \\
13.4876(2), \\
57.4638(16)\end{array}$ & $\begin{array}{l}15.2134(2), \\
13.99260(17), \\
16.96049(17)\end{array}$ \\
\hline$\alpha, \beta, \gamma\left({ }^{\circ}\right)$ & $90,90,120$ & $90,98.3027(12), 90$ \\
\hline$V\left(\AA^{3}\right)$ & $9053.0(4)$ & $3572.61(7)$ \\
\hline$Z$ & 12 & 4 \\
\hline Radiation type & \multicolumn{2}{|c|}{$\mathrm{Cu} K \alpha$} \\
\hline$\mu\left(\mathrm{mm}^{-1}\right)$ & 1.24 & 2.65 \\
\hline Crystal size (mm) & $0.28 \times 0.19 \times 0.07$ & $0.49 \times 0.05 \times 0.02$ \\
\hline Diffractometer & \multicolumn{2}{|c|}{ Oxford Diffraction Xcalibur, Ruby, Gemini Ultra } \\
\hline Absorption correction & \multicolumn{2}{|c|}{ Analytical } \\
\hline$T_{\min }, T_{\max }$ & $0.802,0.917$ & $0.552,0.946$ \\
\hline $\begin{array}{l}\text { No. of measured, } \\
\text { independent and } \\
\text { observed }[I>2 \sigma(I)] \\
\text { reflections }\end{array}$ & $24564,1799,1529$ & $19970,6330,5013$ \\
\hline$R_{\text {int }}$ & 0.037 & 0.032 \\
\hline$(\sin \theta / \lambda)_{\max }\left(\AA^{-1}\right)$ & 0.597 & 0.597 \\
\hline $\begin{array}{l}R\left[F^{2}>2 \sigma\left(F^{2}\right)\right] \\
w R\left(F^{2}\right), S\end{array}$ & $0.065,0.212,1.08$ & $0.043,0.124,1.03$ \\
\hline No. of reflections & 1799 & 6330 \\
\hline No. of parameters & 133 & 599 \\
\hline No. of restraints & 0 & 96 \\
\hline $\mathrm{H}$-atom treatment & \multicolumn{2}{|c|}{ H-atom parameters constrained } \\
\hline$\Delta \rho_{\max }, \Delta \rho_{\min }\left(\mathrm{e} \AA^{-3}\right)$ & $0.56,-0.42$ & $0.36,-0.25$ \\
\hline $\begin{array}{l}\text { CCDC deposition } \\
\text { number }\end{array}$ & 1905042 & 1905043 \\
\hline
\end{tabular}




\section{Computational studies}

\subsection{Methods}

As described by N. Fey ${ }_{1}^{18}$ the $\mathrm{He}_{8}$ steric parameters was computed by optimizing the phosphatriptycene (or $\mathrm{PPh}_{3}$ ) geometry with its bridge-head phosphorus atom constrained to lie at $2.28 \AA$ above the centroid, and perpendicular to the plane, of a helium ring which is constituted by eight helium atoms with a $2.5 \AA$ radius (Figure S9).

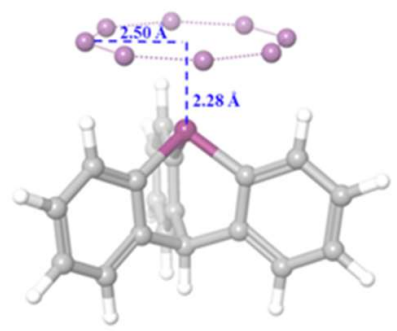

Figure S9. Geometric model used for computation of the He8_steric parameter (helium atoms in violet and phosphorus atom in dark purple).

NCI calculations were carried out using the free open-source program IGMPlot $1.0 .^{5}$ Results were visualized using VMD software. ${ }^{6}$

\subsection{NCI analysis}

We have analyzed non-covalent interactions ( $\mathrm{NCI})$ in $\mathbf{1 8}$ and $\mathbf{2 4}$ Lewis adducts using IGMPlot.Erreur ! Signet non défini. Obtained plots indicate that, in the Lewis adduct between 1 and $B\left(\mathrm{C}_{6} \mathrm{~F}_{5}\right)_{3}(\mathbf{1 8})$, each $\mathrm{C}-\mathrm{H}$ of the phosphatriptycene pointing forward is involved in both a $\mathrm{C}-\mathrm{H} \cdots \mathrm{F}$ hydrogen bond and a $\mathrm{C}-\mathrm{H} \cdots \pi$ interaction (Figure S10). This is made possible by the rigid $\mathrm{C} 3$ structure of the triptycene presenting three $\mathrm{C}-\mathrm{H}$ pointing toward the Lewis acid and the staggered conformation of the three fluorinated aromatic rings of $\mathrm{B}\left(\mathrm{C}_{6} \mathrm{~F}_{5}\right)_{3}$. In the case of $\mathrm{PPh}_{3}$ (adduct 24), the situation is different: the three aromatic rings on the phosphine adopt also a helical conformation (to decrease the steric clashes between these three aromatic rings) and the resulting double helix geometry does not allow efficient $\mathrm{C}-\mathrm{H} \cdots \pi$ interaction between the $\mathrm{C}-\mathrm{H}$ of the phosphine rings and the aromatic $\mathrm{C}_{6} \mathrm{~F}_{5}$ rings of the Lewis acid. The increased stabilization by noncovalent interactions between the two partners in the case of $\mathbf{1}$ is also reflected by the shorter P-B bond length in the complex ( $2.127 \AA$ and $2.257 \AA$ in $\mathbf{1 8}$ and 24, respectively). A NBO analysis of $\mathbf{1 8}$ and $\mathbf{2 4}$ revealed no significant differences in bonding interactions or conjugation effects. 


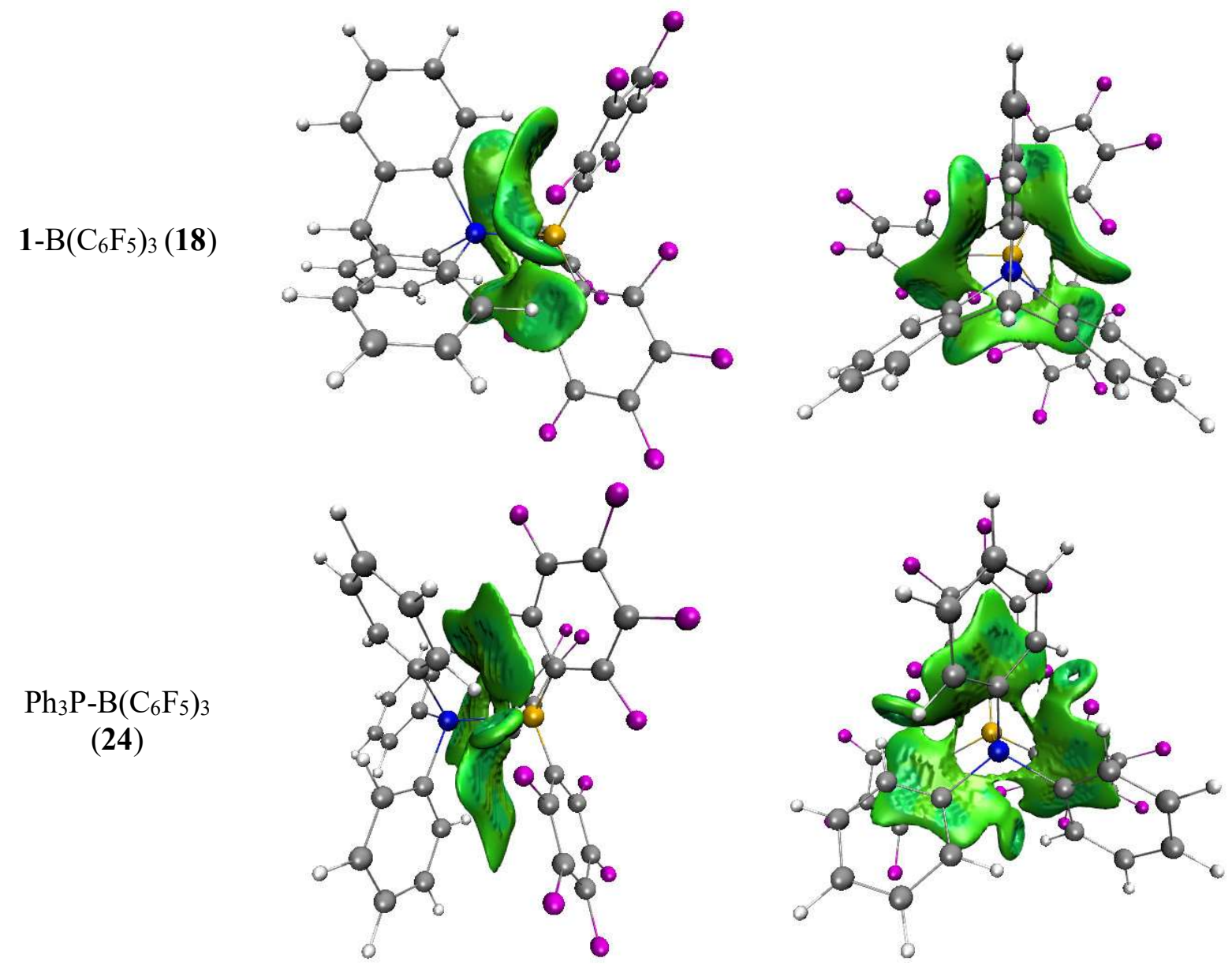

Figure S10. NCI plots for Lewis adducts 18 and 24. 
4.3 Calculations of proton affinities (PA) of triarylphosphines and correlation with $\mathrm{p} K_{\mathrm{a}}$

The proton affinity of weak phosphines has been evaluated based on the energy difference between protonated and non-protonated species in Table S4. The correlation between computed PA and experimental $\mathrm{p} K_{\mathrm{a}}{ }^{7}$ (Figure $\left.\mathrm{S} 11\right)$ was used to estimate the $\mathrm{p} K_{\mathrm{a}}$ of 9-phosphatriptycene (1).

Table S4: Computed (B3LYP-D3/6-31+G*(benzene)) proton affinities (PA) and $\mathrm{p} K_{\mathrm{a}}$ for triarylphosphines.

Lewis Base

PA

$(\mathrm{kcal} / \mathrm{mol})$

$\mathrm{p} K_{\mathrm{a}}^{7}$

$\left(\mathrm{H}_{2} \mathrm{O}\right)$

\begin{tabular}{c|c|c}
\hline P-triptycene & 245.20 & $-1.51^{\mathrm{a}}$ \\
\hline $\mathrm{PPh}_{3}$ & 260.40 & 3.28 \\
\hline $\mathrm{P}\left(p-\mathrm{Cl}-\mathrm{C}_{6} \mathrm{H}_{4}\right)_{3}$ & 252.93 & 0.87 \\
\hline $\mathrm{P}\left(p-\mathrm{CF}_{3}-\mathrm{C}_{6} \mathrm{H}_{4}\right)_{3}$ & 245.49 & -1.39 \\
\hline
\end{tabular}

${ }^{a}$ Estimated using the correlation between computed PA and experimental $\mathrm{p} K_{\mathrm{a}}$ (see Figure S11).

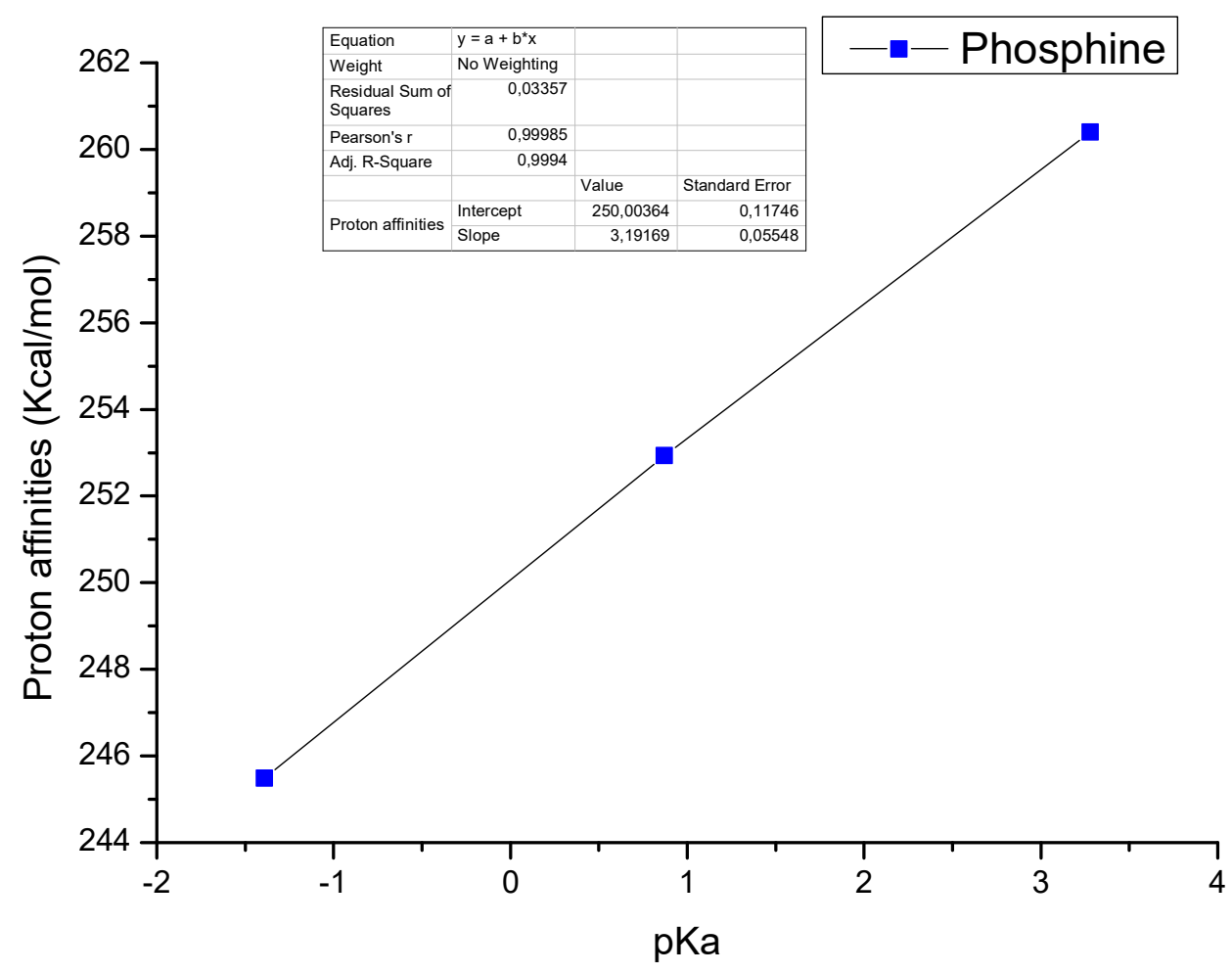

Figure S11: Proton affinities of triarylphosphines and correlation with their $\mathrm{p} K_{\mathrm{a}}$. 


\subsection{Structures and relative energies}

$\mathrm{CH}_{3}^{+}$

$\mathrm{E}\left(\mathrm{B} 3 \mathrm{LYP}-\mathrm{D} 3 / 6-31+\mathrm{G}^{*}\right)($ benzene $)=-39.56224648223$

$\mathrm{E}\left(\mathrm{M} 06-2 \mathrm{X} / 6-311+\mathrm{G}^{* *}\right)=-39.46271278157$

$\mathrm{G}\left(\mathrm{M} 06-2 \mathrm{X} / 6-311+\mathrm{G}^{* *}\right)($ benzene $)=-39.531650247$

$\begin{array}{llll}\mathrm{C} 1 & 3.0866252223 & 1.9106403529 & 7.6382897269 \\ \mathrm{H} 2 & 3.5805951593 & 1.0772588556 & 8.1508386261 \\ \mathrm{H} 3 & 3.0902029343 & 1.9612997196 & 6.5427424919 \\ \mathrm{H} 4 & 2.5892993326 & 2.6938573623 & 8.2232182273\end{array}$

$\mathrm{CPh}_{3}{ }^{+}$

$\mathrm{E}\left(\mathrm{B} 3 \mathrm{LYP}-\mathrm{D} 3 / 6-31+\mathrm{G}^{*}\right)($ benzene $)=-732.90972733517$

$\mathrm{E}\left(\mathrm{M} 06-2 \mathrm{X} / 6-311+\mathrm{G}^{* *}\right)=-732.66902424505$

$\mathrm{G}\left(\mathrm{M} 06-2 \mathrm{X} / 6-311+\mathrm{G}^{* *}\right)($ benzene $)=-732.467685419$

$\begin{array}{lccc}\text { C1 } & -1.3227996983 & -0.3575972039 & 1.6590835122 \\ \text { C2 } & -1.7091340473 & -1.5106156789 & 2.3946879933 \\ \text { C3 } & -0.6918418267 & 0.7131998704 & 2.3484706093 \\ \text { C4 } & -1.4849304262 & -1.5779032129 & 3.7646756077 \\ \text { C5 } & -0.4424690080 & 0.6217525673 & 3.7128566433 \\ \text { C6 } & -0.8452125834 & -0.5181425322 & 4.4243519765 \\ \text { C7 } & -1.4930268910 & -1.4617510807 & -0.5913441365 \\ \text { C8 } & -2.3576387658 & -1.6286271689 & -1.7062433064 \\ \text { C9 } & -0.5463444038 & -2.4817821303 & -0.3018051743 \\ \text { C10 } & -2.2874268418 & -2.7778740862 & -2.4847700212 \\ \text { C11 } & -0.4627702986 & -3.6121119026 & -1.1065476764 \\ \text { C12 } & -1.3359758882 & -3.7661410144 & -2.1931313600 \\ \text { C13 } & -1.9118668682 & 1.0013098125 & -0.3695412229 \\ \text { C14 } & -2.6871266557 & 1.9563477345 & 0.3426535948 \\ \text { C15 } & -1.4797802350 & 1.3159741924 & -1.6856708930 \\ \text { C16 } & -3.0237736912 & 3.1690206645 & -0.2471367625 \\ \text { C17 } & -1.7957131543 & 2.5441920889 & -2.2539611789 \\ \text { C18 } & -2.5736092066 & 3.4685950698 & -1.5410716122 \\ \text { C19 } & -1.5738538223 & -0.2721195062 & 0.2333233293 \\ \text { H20 } & -0.8533054647 & 0.6138007094 & -2.2277877620 \\ \text { H21 } & -1.4367829147 & 2.7873166139 & -3.2505691766 \\ \text { H22 } & -2.8303110576 & 4.4228058490 & -1.9950954084 \\ \text { H23 } & -3.6416860362 & 3.8806472353 & 0.2941346737 \\ \text { H24 } & -3.0628784632 & 1.7136187682 & 1.3318398951 \\ \text { H25 } & -0.3526298172 & 1.5827706228 & 1.7939531185 \\ \text { H26 } & 0.0691847184 & 1.4320841288 & 4.2255176007 \\ \text { H27 } & -0.6610802571 & -0.5802524630 & 5.4944597552 \\ \text { H28 } & -1.8127144813 & -2.4501788707 & 4.3241949345 \\ \text { H29 } & -2.2319345257 & -2.3185331430 & 1.8924660876 \\ \text { H30 } & 0.1511796332 & -2.3504967505 & 0.5194515009\end{array}$




$\begin{array}{lrrr}\text { H31 } & 0.2839555986 & -4.3729434491 & -0.8948541183 \\ \text { H32 } & -1.2753198599 & -4.6574762283 & -2.8132239723 \\ \text { H33 } & -2.9735692349 & -2.9104564631 & -3.3171706584 \\ \text { H34 } & -3.1130958808 & -0.8775947481 & -1.9163660596\end{array}$

$\mathbf{B F}_{3}$

E(B3LYP-D3/6-31+G*) (benzene $)=-324.58218545607$

$\mathrm{E}\left(\mathrm{M} 06-2 \mathrm{X} / 6-311+\mathrm{G}^{* *}\right)=-324.55965730812$

$\mathrm{G}\left(\mathrm{M} 06-2 \mathrm{X} / 6-311+\mathrm{G}^{* *}\right)($ benzene $)=-324.575474865$

$\begin{array}{llll}\text { B1 } & 3.8411388286 & 2.3936308426 & 7.6660774292 \\ \text { F2 } & 4.5035368075 & 1.3582695031 & 7.1780356649 \\ \text { F3 } & 3.3106469940 & 3.2852916782 & 6.8461200030 \\ \text { F4 } & 3.7078078119 & 2.5364067542 & 8.9739907537\end{array}$

$\mathbf{B}\left(\mathbf{C}_{6} \mathbf{F}_{5}\right)_{3}$

$\mathrm{E}\left(\mathrm{B} 3 \mathrm{LYP}-\mathrm{D} 3 / 6-31+\mathrm{G}^{*}\right)($ benzene $)=-2208.41192445835$

$\mathrm{E}\left(\mathrm{M} 06-2 \mathrm{X} / 6-311+\mathrm{G}^{* *}\right)=-2208.20818717732$

$\mathrm{G}\left(\mathrm{M} 06-2 \mathrm{X} / 6-311+\mathrm{G}^{* *}\right)($ benzene $)=-2208.120487580$

$\begin{array}{llll}\text { C1 } & -1.3533627505 & -0.5786626332 & 1.7448439662 \\ \text { C2 } & -1.7880963160 & -1.7435551742 & 2.3944411206 \\ \text { C3 } & -0.6255340753 & 0.3193754506 & 2.5395965888 \\ \text { C4 } & -1.5343672536 & -2.0027014683 & 3.7359190820 \\ \text { C5 } & -0.3346963397 & 0.0837075469 & 3.8776735257 \\ \text { C6 } & -0.7977512473 & -1.0839663431 & 4.4807624643 \\ \text { C7 } & -1.6367011010 & -1.4700550885 & -0.8030370104 \\ \text { C8 } & -2.5546512477 & -1.5550925687 & -1.8603705497 \\ \text { C9 } & -0.6914486763 & -2.5046609661 & -0.7397051277 \\ \text { C10 } & -2.5519864719 & -2.5925715815 & -2.7848243715 \\ \text { C11 } & -0.6443762606 & -3.5453171831 & -1.6594122672 \\ \text { C12 } & -1.5855839910 & -3.5916611292 & -2.6858574774 \\ \text { C13 } & -2.0023878042 & 1.1585762316 & -0.2359866817 \\ \text { C14 } & -2.7784105824 & 2.0346879744 & 0.5373143178 \\ \text { C15 } & -1.5560407955 & 1.6711924688 & -1.4630876603 \\ \text { C16 } & -3.1020422549 & 3.3225454259 & 0.1280401092 \\ \text { C17 } & -1.8413526659 & 2.9607841615 & -1.8948879302 \\ \text { C18 } & -2.6251890866 & 3.7900457286 & -1.0950107428 \\ \text { B19 } & -1.6633656620 & -0.2969914284 & 0.2352200303 \\ \text { F20 } & -0.7887101385 & 0.9126567705 & -2.2722705563 \\ \text { F21 } & -1.3768509492 & 3.4119328106 & -3.0690663397 \\ \text { F22 } & -2.9179970349 & 5.0280541599 & -1.4989534673 \\ \text { F23 } & -3.8613331758 & 4.1177462483 & 0.8955457592 \\ \text { F24 } & -3.2725508776 & 1.6306447870 & 1.7251358810 \\ \text { F25 } & -0.1481975168 & 1.4614621727 & 2.0046340371 \\ \text { F26 } & 0.3792243787 & 0.9650804498 & 4.5926333579\end{array}$




$\begin{array}{lccc}\text { F27 } & -0.5361933994 & -1.3217755791 & 5.7678685659 \\ \text { F28 } & -1.9849272113 & -3.1236073642 & 4.3176067416 \\ \text { F29 } & -2.5113789629 & -2.6624181096 & 1.7231176739 \\ \text { F30 } & 0.2461395535 & -2.5049145061 & 0.2292894317 \\ \text { F31 } & 0.2893579643 & -4.5030983628 & -1.5676759910 \\ \text { F32 } & -1.5619007060 & -4.5900630284 & -3.5714443411 \\ \text { F33 } & -3.4617328461 & -2.6403346026 & -3.7686268231 \\ \text { F34 } & -3.5142901324 & -0.6178054537 & -1.9982196954 \\ \text { C1 } & -1.3533627505 & -0.5786626332 & 1.7448439662 \\ \text { C2 } & -1.7880963160 & -1.7435551742 & 2.3944411206 \\ \text { C3 } & -0.6255340753 & 0.3193754506 & 2.5395965888 \\ \text { C4 } & -1.5343672536 & -2.0027014683 & 3.7359190820 \\ \text { C5 } & -0.3346963397 & 0.0837075469 & 3.8776735257 \\ \text { C6 } & -0.7977512473 & -1.0839663431 & 4.4807624643 \\ \text { C7 } & -1.6367011010 & -1.4700550885 & -0.8030370104 \\ \text { C8 } & -2.5546512477 & -1.5550925687 & -1.8603705497 \\ \text { C9 } & -0.6914486763 & -2.5046609661 & -0.7397051277 \\ \text { C10 } & -2.5519864719 & -2.5925715815 & -2.7848243715 \\ \text { C11 } & -0.6443762606 & -3.5453171831 & -1.6594122672 \\ \text { C12 } & -1.5855839910 & -3.5916611292 & -2.6858574774 \\ \text { C13 } & -2.0023878042 & 1.1585762316 & -0.2359866817 \\ \text { C14 } & -2.7784105824 & 2.0346879744 & 0.5373143178 \\ \text { C15 } & -1.5560407955 & 1.6711924688 & -1.4630876603 \\ \text { C16 } & -3.1020422549 & 3.3225454259 & 0.1280401092 \\ \text { C17 } & -1.8413526659 & 2.9607841615 & -1.8948879302 \\ \text { C18 } & -2.6251890866 & 3.7900457286 & -1.0950107428 \\ \text { B19 } & -1.6633656620 & -0.2969914284 & 0.2352200303 \\ \text { F20 } & -0.7887101385 & 0.9126567705 & -2.2722705563 \\ \text { F21 } & -1.3768509492 & 3.4119328106 & -3.0690663397 \\ \text { F22 } & -2.9179970349 & 5.0280541599 & -1.4989534673 \\ \text { F23 } & -3.8613331758 & 4.1177462483 & 0.8955457592 \\ \text { F24 } & -3.2725508776 & 1.6306447870 & 1.7251358810 \\ \text { F25 } & -0.1481975168 & 1.4614621727 & 2.0046340371 \\ \text { F26 } & 0.3792243787 & 0.9650804498 & 4.5926333579 \\ \text { F27 } & -0.5361933994 & -1.3217755791 & 5.7678685659 \\ \text { F28 } & -1.9849272113 & -3.1236073642 & 4.3176067416 \\ \text { F29 } & -2.5113789629 & -2.6624181096 & 1.7231176739 \\ \text { F30 } & 0.2461395535 & -2.5049145061 & 0.2292894317 \\ \text { F31 } & 0.2893579643 & -4.5030983628 & -1.5676759910 \\ \text { F32 } & -1.5619007060 & -4.5900630284 & -3.5714443411 \\ \text { F33 } & -3.4617328461 & -2.6403346026 & -3.7686268231 \\ \text { F34 } & -3.5142901324 & -0.6178054537 & -1.9982196954\end{array}$

$\mathrm{BPh}_{3}$

$\mathrm{E}\left(\mathrm{B} 3 \mathrm{LYP}-\mathrm{D} 3 / 6-31+\mathrm{G}^{*}\right)($ benzene $)=-719.88195080865$

$\mathrm{E}\left(\mathrm{M} 06-2 \mathrm{X} / 6-311+\mathrm{G}^{* *}\right)=-719.6907205$

$\mathrm{G}\left(\mathrm{M} 06-2 \mathrm{X} / 6-311+\mathrm{G}^{* *}\right)($ benzene $)=-719.4599851$ 


$\begin{array}{llrl}\text { C1 } & -1.3136057814 & -0.3618602436 & 1.7689544020 \\ \text { C2 } & -1.6622751801 & -1.5106159623 & 2.5148344934 \\ \text { C3 } & -0.7099540642 & 0.7051121835 & 2.4723735942 \\ \text { C4 } & -1.4350923819 & -1.5864187985 & 3.8909577974 \\ \text { C5 } & -0.4511188179 & 0.6248546629 & 3.8427978345 \\ \text { C6 } & -0.8208136235 & -0.5202156185 & 4.5576241866 \\ \text { C7 } & -1.4953004784 & -1.5653306222 & -0.6597277596 \\ \text { C8 } & -2.3211551277 & -1.7467956038 & -1.7919212659 \\ \text { C9 } & -0.5809821787 & -2.5995079319 & -0.3560028999 \\ \text { C10 } & -2.2507300592 & -2.9039353828 & -2.5711749028 \\ \text { C11 } & -0.4837287630 & -3.7463574617 & -1.1471845328 \\ \text { C12 } & -1.3249037425 & -3.9046362721 & -2.2543979654 \\ \text { C13 } & -1.9487508327 & 1.1077033285 & -0.4231489060 \\ \text { C14 } & -2.6813489109 & 2.0853587583 & 0.2872574472 \\ \text { C15 } & -1.5585877755 & 1.4275684966 & -1.7433099355 \\ \text { C16 } & -3.0193107733 & 3.3108759817 & -0.2913369268 \\ \text { C17 } & -1.8664999325 & 2.6625754925 & -2.3184998006 \\ \text { C18 } & -2.6054961521 & 3.6058440965 & -1.5952917558 \\ \text { B19 } & -1.5873127889 & -0.2738581108 & 0.2261339800 \\ \text { H20 } & -0.9920804470 & 0.7010252285 & -2.3205919403 \\ \text { H21 } & -1.5385949825 & 2.8878156201 & -3.3313228415 \\ \text { H22 } & -2.8577212464 & 4.5639960322 & -2.0450429514 \\ \text { H23 } & -3.5989588179 & 4.0377626414 & 0.2740984597 \\ \text { H24 } & -3.0021615735 & 1.8736944474 & 1.3043809304 \\ \text { H25 } & -0.4261840805 & 1.6047361415 & 1.9313229637 \\ \text { H26 } & 0.0310927258 & 1.4548440846 & 4.3552669315 \\ \text { H27 } & -0.6319137850 & -0.5813242983 & 5.6274879615 \\ \text { H28 } & -1.7299912698 & -2.4767770370 & 4.4427573439 \\ \text { H29 } & -2.1315663211 & -2.3508084690 & 2.0086803569 \\ \text { H30 } & 0.0725508062 & -2.4944510541 & 0.5066817137 \\ \text { H31 } & 0.2408433765 & -4.5192722222 & -0.8987234069 \\ \text { H32 } & -1.2597004633 & -4.8021953276 & -2.8661556997 \\ \text { H33 } & -2.9106869663 & -3.0239297513 & -3.4280122308 \\ \text { H34 } & -3.0394386105 & -0.9741990828 & -2.0552346663 \\ & & & \end{array}$

\section{$\mathbf{P P h}_{3}$}

$\mathrm{E}\left(\mathrm{B} 3 \mathrm{LYP}-\mathrm{D} 3 / 6-31+\mathrm{G}^{*}\right)($ benzene $)=-1036.35400266933$

$\mathrm{E}\left(\mathrm{M} 06-2 \mathrm{X} / 6-311+\mathrm{G}^{* *}\right)=-1036.1647753062$

$\mathrm{G}\left(\mathrm{M} 06-2 \mathrm{X} / 6-311+\mathrm{G}^{* *}\right)($ benzene $)=-1035.941852420$

$\begin{array}{llrr}\text { C1 } & -0.9738170388 & -0.2796172423 & 1.8224800605 \\ \text { C2 } & -1.9253873044 & -1.2165023449 & 2.2565807818 \\ \text { C3 } & -0.3653964340 & 0.5577818031 & 2.7744888142 \\ \text { C4 } & -2.2621428459 & -1.3108673675 & 3.6112423169 \\ \text { C5 } & -0.7109521217 & 0.4722552334 & 4.1255885953 \\ \text { C6 } & -1.6599890123 & -0.4656369062 & 4.5480821737 \\ \text { C7 } & -1.1359308896 & -1.5971393698 & -0.7389979042 \\ \text { C8 } & -2.4014890450 & -1.6661204514 & -1.3441863552 \\ \text { C9 } & -0.3231547002 & -2.7445348401 & -0.7395192368\end{array}$




$\begin{array}{lrrr}\text { C10 } & -2.8429750662 & -2.8553314774 & -1.9341040918 \\ \text { C11 } & -0.7691037204 & -3.9358825017 & -1.3177514775 \\ \text { C12 } & -2.0308717460 & -3.9934839405 & -1.9198447187 \\ \text { C13 } & -1.6036379976 & 1.2413700334 & -0.5412128235 \\ \text { C14 } & -2.7455134214 & 1.6652834263 & 0.1575915949 \\ \text { C15 } & -1.3025250737 & 1.8449090197 & -1.7749672456 \\ \text { C16 } & -3.5686080445 & 2.6669053418 & -0.3680550510 \\ \text { C17 } & -2.1313399951 & 2.8367532479 & -2.3057986201 \\ \text { C18 } & -3.2669337776 & 3.2522669490 & -1.6014268683 \\ \text { P19 } & -0.4427510159 & -0.0732763414 & 0.0588809574 \\ \text { H20 } & -0.4121360286 & 1.5406809663 & -2.3232461821 \\ \text { H21 } & -1.8856196712 & 3.2915031630 & -3.2631031102 \\ \text { H22 } & -3.9078135894 & 4.0316141570 & -2.0078779660 \\ \text { H23 } & -4.4474938187 & 2.9881649154 & 0.1872353111 \\ \text { H24 } & -2.9942382352 & 1.2180981252 & 1.1164187455 \\ \text { H25 } & 0.3866927108 & 1.2793255582 & 2.4586862269 \\ \text { H26 } & -0.2329071286 & 1.1294964082 & 4.8487866715 \\ \text { H27 } & -1.9226956359 & -0.5419199744 & 5.6009446590 \\ \text { H28 } & -2.9973775250 & -2.0455305203 & 3.9333691842 \\ \text { H29 } & -2.4039582942 & -1.8786230034 & 1.5398603198 \\ \text { H30 } & 0.6677116183 & -2.7059225088 & -0.2894941647 \\ \text { H31 } & -0.1280746668 & -4.8148292095 & -1.3078589394 \\ \text { H32 } & -2.3752411714 & -4.9165251963 & -2.3812098239 \\ \text { H33 } & -3.8233935474 & -2.8914218196 & -2.4048216371 \\ \text { H34 } & -3.0448369537 & -0.7905981960 & -1.3626387201\end{array}$

$\mathbf{P P h}_{3}+\mathbf{H}^{+}$

$\mathrm{E}\left(\mathrm{B} 3 \mathrm{LYP}-\mathrm{D} 3 / 6-31+\mathrm{G}^{*}\right)($ benzene $)=-1036.73510875599$

$\begin{array}{llcl}\text { C1 } & -0.9351703562 & -0.2889575391 & 1.8664225890 \\ \text { C2 } & -1.7952797315 & -1.2795767020 & 2.3678990837 \\ \text { C3 } & -0.3029409387 & 0.6205368652 & 2.7339737959 \\ \text { C4 } & -2.0221971096 & -1.3558168439 & 3.7441374702 \\ \text { C5 } & -0.5350146602 & 0.5301621325 & 4.1066506180 \\ \text { C6 } & -1.3945199730 & -0.4546174104 & 4.6098787353 \\ \text { C7 } & -1.1320429231 & -1.6400893190 & -0.7798705199 \\ \text { C8 } & -2.3616588286 & -1.7348368447 & -1.4524231580 \\ \text { C9 } & -0.2588442527 & -2.7417032814 & -0.7205840831 \\ \text { C10 } & -2.7153560669 & -2.9388617436 & -2.0659183598 \\ \text { C11 } & -0.6232930360 & -3.9370574418 & -1.3411464843 \\ \text { C12 } & -1.8496234607 & -4.0358087604 & -2.0099875306 \\ \text { C13 } & -1.5964428142 & 1.2976063686 & -0.5654631971 \\ \text { C14 } & -2.7515708209 & 1.7539569173 & 0.0918281596 \\ \text { C15 } & -1.1679358086 & 1.8962872436 & -1.7639366431 \\ \text { C16 } & -3.4797376570 & 2.8107882623 & -0.4589150283 \\ \text { C17 } & -1.9028933686 & 2.9543214008 & -2.3005190323 \\ \text { C18 } & -3.0569995113 & 3.4086609611 & -1.6508632616 \\ \text { H19 } & -0.2739415486 & 1.5456997702 & -2.2764911262 \\ \text { H20 } & -1.5739994900 & 3.4253647837 & -3.2231815620 \\ \text { H21 } & -3.6249300514 & 4.2341679241 & -2.0729548069 \\ \text { H22 } & -4.3730101541 & 3.1689461114 & 0.0463246239\end{array}$




$\begin{array}{lrrr}\text { H23 } & -3.0781132229 & 1.3005635724 & 1.0244208498 \\ \text { H24 } & 0.3616142587 & 1.3920720102 & 2.3491861781 \\ \text { H25 } & -0.0458285938 & 1.2261631517 & 4.7831363619 \\ \text { H26 } & -1.5717575241 & -0.5207687040 & 5.6805635305 \\ \text { H27 } & -2.6861753662 & -2.1211855310 & 4.1376684384 \\ \text { H28 } & -2.2788097834 & -1.9880603982 & 1.7002521627 \\ \text { H29 } & 0.6942119481 & -2.6738138318 & -0.1991361434 \\ \text { H30 } & 0.0489896468 & -4.7902915417 & -1.3042083639 \\ \text { H31 } & -2.1279011925 & -4.9696785174 & -2.4923927573 \\ \text { H32 } & -3.6645859594 & -3.0168920774 & -2.5897561153 \\ \text { H33 } & -3.0336496895 & -0.8822801836 & -1.5076184818 \\ \text { P34 } & -0.6911278191 & -0.1199210299 & 0.0867113895 \\ \text { H35 } & 0.6766608303 & 0.1163949310 & -0.1393260845\end{array}$

$\left(p-\mathrm{Cl}-\mathrm{C}_{6} \mathrm{H}_{5}\right)_{3} \mathrm{P}$

$\begin{array}{lccc}\text { E(B3LYP-D3/6-31+G*)(benzene })=-2415.14730636408 & \\ \text { C1 } & -0.9481149308 & -0.2758152932 & 1.8124822442 \\ \text { C2 } & -1.8965439786 & -1.2159781448 & 2.2443660410 \\ \text { C3 } & -0.3471408337 & 0.5619950649 & 2.7682367236 \\ \text { C4 } & -2.2463536831 & -1.3163000711 & 3.5934746392 \\ \text { C5 } & -0.6951997153 & 0.4830999959 & 4.1173861944 \\ \text { C6 } & -1.6441440959 & -0.4602669943 & 4.5151617797 \\ \text { C7 } & -1.1112755857 & -1.5910740160 & -0.7417686977 \\ \text { C8 } & -2.3843884004 & -1.6615000391 & -1.3287805490 \\ \text { C9 } & -0.2978809430 & -2.7374382667 & -0.7581741133 \\ \text { C10 } & -2.8426901038 & -2.8459741379 & -1.9110710469 \\ \text { C11 } & -0.7459046530 & -3.9317422938 & -1.3249321630 \\ \text { C12 } & -2.0181708181 & -3.9708870033 & -1.8977117947 \\ \text { C13 } & -1.5778968539 & 1.2405945279 & -0.5490544151 \\ \text { C14 } & -2.7286252911 & 1.6506878132 & 0.1421674169 \\ \text { C15 } & -1.2691287247 & 1.8630745985 & -1.7711175048 \\ \text { C16 } & -3.5582330906 & 2.6503205777 & -0.3725836711 \\ \text { C17 } & -2.0944717074 & 2.8538422643 & -2.3049245484 \\ \text { C18 } & -3.2333824372 & 3.2376790633 & -1.5950278519 \\ \text { P19 } & -0.4120406505 & -0.0691173167 & 0.0507761152 \\ \text { H20 } & -0.3733725390 & 1.5754521812 & -2.3190761256 \\ \text { H21 } & -1.8526038486 & 3.3278121283 & -3.2516367403 \\ \text { C122 } & -4.2752243938 & 4.4951623260 & -2.2515810431 \\ \text { H23 } & -4.4460130697 & 2.9653908246 & 0.1681537261 \\ \text { H24 } & -2.9907690347 & 1.1938535241 & 1.0922102919 \\ \text { H25 } & 0.4035672721 & 1.2887715444 & 2.4621789853 \\ \text { H26 } & -0.2306317723 & 1.1362832692 & 4.8501747544 \\ \text { C127 } & -2.0823751377 & -0.5777798735 & 6.2157442059 \\ \text { H28 } & -2.9789816628 & -2.0476967621 & 3.9223221395 \\ \text { H29 } & -2.3739914869 & -1.8820687874 & 1.5314750578 \\ \text { H30 } & 0.7002178688 & -2.7044712903 & -0.3244550751 \\ \text { H31 } & -0.1143751265 & -4.8152058696 & -1.3310876925 \\ \text { C132 } & -2.5896499070 & -5.4674897379 & -2.6265451059 \\ \text { H33 } & -3.8276492087 & -2.8941055341 & -2.3663829318 \\ \text { H34 } & -3.0335639385 & -0.7905847989 & -1.3383968859 \\ & & & \\ & & & \end{array}$




\begin{tabular}{|c|c|c|c|}
\hline \multicolumn{4}{|c|}{$\mathrm{E}\left(\mathrm{B} 3 \mathrm{LYP}-\mathrm{D} 3 / 6-31+\mathrm{G}^{*}\right)($ benzene $)=-2415.51384230201$} \\
\hline $\mathrm{C} 1$ & -0.9172563910 & -0.2824037760 & 1.8580719366 \\
\hline $\mathrm{C} 2$ & -1.7791379766 & -1.2788997979 & 2.3445008021 \\
\hline $\mathrm{C} 3$ & -0.3005159750 & 0.6211478792 & 2.7421831266 \\
\hline $\mathrm{C} 4$ & -2.0322933160 & -1.3695301111 & 3.7122541981 \\
\hline C5 & -0.5496873774 & 0.5302476854 & 4.1089734949 \\
\hline C6 & -1.4162812911 & -0.4635531469 & 4.5801758032 \\
\hline $\mathrm{C} 7$ & -1.0971030191 & -1.6223665542 & -0.7800744366 \\
\hline C8 & -2.3134634632 & -1.7016667302 & -1.4777715083 \\
\hline C9 & -0.2463992546 & -2.7396189428 & -0.7001969340 \\
\hline $\mathrm{C} 10$ & -2.6855150478 & -2.8979867865 & -2.0887354755 \\
\hline C11 & -0.6155795512 & -3.9351781729 & -1.3110558250 \\
\hline $\mathrm{C} 12$ & -1.8340125898 & -4.0028008823 & -1.9975722055 \\
\hline C13 & -1.5718107959 & 1.2989384478 & -0.5636836556 \\
\hline C14 & -2.6812028173 & 1.7964490698 & 0.1397506854 \\
\hline C15 & -1.2079457365 & 1.8518450356 & -1.8047279697 \\
\hline C16 & -3.4323582280 & 2.8401122130 & -0.3981240917 \\
\hline $\mathrm{C} 17$ & -1.9551904197 & 2.8967509057 & -2.3421883951 \\
\hline $\mathrm{C} 18$ & -3.0627474557 & 3.3786325440 & -1.6340090626 \\
\hline H19 & -0.3512204067 & 1.4749554194 & -2.3601480273 \\
\hline $\mathrm{H} 20$ & -1.6842605203 & 3.3349467180 & -3.2979103470 \\
\hline $\mathrm{Cl} 21$ & -4.0008864433 & 4.6850241153 & -2.3087518817 \\
\hline H22 & -4.2922060979 & 3.2324493621 & 0.1364167165 \\
\hline $\mathrm{H} 23$ & -2.9676408456 & 1.3819529293 & 1.1024962054 \\
\hline $\mathrm{H} 24$ & 0.3684209282 & 1.3984973912 & 2.3777637787 \\
\hline H25 & -0.0790513561 & 1.2196874229 & 4.8032069581 \\
\hline $\mathrm{C} 126$ & -1.7312307571 & -0.5749857186 & 6.2918940553 \\
\hline H27 & -2.6975900058 & -2.1351602467 & 4.0997206294 \\
\hline H28 & -2.2566641841 & -1.9859179324 & 1.6716737775 \\
\hline H29 & 0.6986889271 & -2.6913013527 & -0.1626260254 \\
\hline H30 & 0.0314561425 & -4.8053833915 & -1.2570655127 \\
\hline $\mathrm{Cl} 31$ & -2.2982613361 & -5.5008701838 & -2.7603582355 \\
\hline H32 & -3.6232851552 & -2.9714229256 & -2.6310611732 \\
\hline H33 & -2.9751817668 & -0.8429988999 & -1.5519568668 \\
\hline P34 & -0.6519729544 & -0.1070089140 & 0.0853830383 \\
\hline H35 & 0.7171292524 & 0.1310187410 & -0.1312923738 \\
\hline
\end{tabular}

$\left(p-\mathrm{CF}_{3}-\mathrm{C}_{6} \mathrm{H}_{5}\right)_{3} \mathrm{P}$

$\mathrm{E}\left(\mathrm{B} 3 \mathrm{LYP}-\mathrm{D} 3 / 6-31+\mathrm{G}^{*}\right)($ benzene $)=-2047.54643809047$

$\begin{array}{lrrr}\text { C1 } & -0.9252747126 & -0.2787086824 & 1.8274386526 \\ \text { C2 } & -1.8733611692 & -1.2220729598 & 2.2545384815 \\ \text { C3 } & -0.3213664148 & 0.5572375850 & 2.7823273748 \\ \text { C4 } & -2.2143517238 & -1.3252975064 & 3.6035277205 \\ \text { C5 } & -0.6672968657 & 0.4687071218 & 4.1309033241 \\ \text { C6 } & -1.6105819805 & -0.4790428140 & 4.5387702317 \\ \text { C7 } & -1.0760148770 & -1.5912290131 & -0.7384376057 \\ \text { C8 } & -2.3180613811 & -1.6442620986 & -1.3913917410\end{array}$




$\begin{array}{lccc}\text { C9 } & -0.2782903690 & -2.7479583823 & -0.7023241693 \\ \text { C10 } & -2.7541188281 & -2.8245621645 & -1.9943138140 \\ \text { C11 } & -0.7139622739 & -3.9352758376 & -1.2909847248 \\ \text { C12 } & -1.9502678947 & -3.9674260512 & -1.9434792833 \\ \text { C13 } & -1.5614998274 & 1.2366991093 & -0.5408949374 \\ \text { C14 } & -2.7194512648 & 1.6354995232 & 0.1455620906 \\ \text { C15 } & -1.2512152519 & 1.8533789638 & -1.7650890086 \\ \text { C16 } & -3.5551996890 & 2.6198956733 & -0.3832959866 \\ \text { C17 } & -2.0884195364 & 2.8289914055 & -2.3059377289 \\ \text { C18 } & -3.2419781900 & 3.2080750351 & -1.6124724887 \\ \text { P19 } & -0.3874318408 & -0.0663459413 & 0.0644284014 \\ \text { H20 } & -0.3503923525 & 1.5696927530 & -2.3059854311 \\ \text { H21 } & -1.8430612104 & 3.2917202494 & -3.2573426163 \\ \text { H22 } & -4.4509661782 & 2.9184785777 & 0.1542933775 \\ \text { H23 } & -2.9812527477 & 1.1773048916 & 1.0941698688 \\ \text { H24 } & 0.4284600459 & 1.2837968291 & 2.4751909125 \\ \text { H25 } & -0.1949208621 & 1.1212930258 & 4.8590402499 \\ \text { H26 } & -2.9464154700 & -2.0618573585 & 3.9237205613 \\ \text { H27 } & -2.3522902921 & -1.8823717486 & 1.5381390190 \\ \text { H28 } & 0.6949090695 & -2.7249391962 & -0.2156334057 \\ \text { H29 } & -0.0885103219 & -4.8223379333 & -1.2566838414 \\ \text { H30 } & -3.7137710601 & -2.8521154116 & -2.5033430813 \\ \text { H31 } & -2.9530726160 & -0.7649807439 & -1.4378306510 \\ \text { C32 } & -2.0236585814 & -0.5695398189 & 5.9815887849 \\ \text { F33 } & -1.0995998908 & -0.0475167256 & 6.8249664243 \\ \text { F34 } & -3.1873643748 & 0.1043665334 & 6.2162898063 \\ \text { F35 } & -2.2419949653 & -1.8519425046 & 6.3736805595 \\ \text { C36 } & -4.1261634355 & 4.2952616448 & -2.1570254512 \\ \text { F37 } & -4.0347669967 & 4.4130220237 & -3.5054834737 \\ \text { F38 } & -5.4362150432 & 4.0891497371 & -1.8619314239 \\ \text { F39 } & -3.8053618841 & 5.5162205779 & -1.6396580816 \\ \text { C40 } & -2.4498061669 & -5.2445273148 & -2.5599677701 \\ \text { F41 } & -1.4469550769 & -6.1052472876 & -2.8642336978 \\ \text { F42 } & -3.2940377716 & -5.9137407480 & -1.7217050692 \\ \text { F43 } & -3.1476746847 & -5.0240575151 & -3.7044719212\end{array}$

\section{$\left(p-\mathrm{CF}_{3}-\mathrm{C}_{6} \mathrm{H}_{5}\right)_{3} \mathbf{P}+\mathbf{H}^{+}$}

$\mathrm{E}\left(\mathrm{B} 3 \mathrm{LYP}-\mathrm{D} 3 / 6-31+\mathrm{G}^{*}\right)($ benzene $)=-2047.89721065282$

$\begin{array}{llcl}\text { C1 } & -0.8866662644 & -0.2775449444 & 1.8535529881 \\ \text { C2 } & -1.8443171328 & -1.1951769045 & 2.3167832485 \\ \text { C3 } & -0.1877043968 & 0.5484143032 & 2.7488736113 \\ \text { C4 } & -2.1072918231 & -1.2791818249 & 3.6829382918 \\ \text { C5 } & -0.4535905104 & 0.4566233862 & 4.1146051708 \\ \text { C6 } & -1.4107947025 & -0.4545855981 & 4.5724212166 \\ \text { C7 } & -1.0478835044 & -1.6137546400 & -0.7960983316 \\ \text { C8 } & -2.3127947657 & -1.7300971307 & -1.3960938907 \\ \text { C9 } & -0.1461676103 & -2.6905234752 & -0.7999519859 \\ \text { C10 } & -2.6760220071 & -2.9332649416 & -1.9993514520 \\ \text { C11 } & -0.5144370324 & -3.8891970239 & -1.4091987178\end{array}$




$\begin{array}{lrrr}\text { C12 } & -1.7749428593 & -4.0029816238 & -2.0045538403 \\ \text { C13 } & -1.5408144938 & 1.3083034090 & -0.5730411013 \\ \text { C14 } & -2.6272177685 & 1.8243799425 & 0.1517226010 \\ \text { C15 } & -1.2001818013 & 1.8322978336 & -1.8314831469 \\ \text { C16 } & -3.3757471619 & 2.8696903402 & -0.3878440807 \\ \text { C17 } & -1.9502943934 & 2.8802570189 & -2.3625402602 \\ \text { C18 } & -3.0312706123 & 3.3933089626 & -1.6373002192 \\ \text { H19 } & -0.3631583400 & 1.4343797701 & -2.4013737877 \\ \text { H20 } & -1.6909090436 & 3.2962467819 & -3.3311497098 \\ \text { H21 } & -4.2164585428 & 3.2753634226 & 0.1675836553 \\ \text { H22 } & -2.8962121694 & 1.4257665520 & 1.1256612606 \\ \text { H23 } & 0.5560539576 & 1.2605047680 & 2.3975044939 \\ \text { H24 } & 0.0853515846 & 1.0866931914 & 4.8153875292 \\ \text { H25 } & -2.8445858998 & -1.9875964361 & 4.0496501547 \\ \text { H26 } & -2.3834610287 & -1.8425597820 & 1.6309485146 \\ \text { H27 } & 0.8337605929 & -2.6086306933 & -0.3344325186 \\ \text { H28 } & 0.1778056354 & -4.7253502325 & -1.4234460548 \\ \text { H29 } & -3.6514628265 & -3.0315292198 & -2.4670509395 \\ \text { H30 } & -3.0135012222 & -0.8997154049 & -1.4003928480 \\ \text { P31 } & -0.6103370799 & -0.0962251700 & 0.0785533608 \\ \text { H32 } & 0.7596995152 & 0.1437569862 & -0.1249706550 \\ \text { C33 } & -2.2000155771 & -5.3147003566 & -2.6262877719 \\ \text { F34 } & -1.1432404025 & -6.0705468224 & -2.9952735617 \\ \text { F35 } & -2.9325347531 & -6.0485064437 & -1.7481480209 \\ \text { F36 } & -2.9707869797 & -5.1226174559 & -3.7214546099 \\ \text { C37 } & -1.7422464914 & -0.5275649533 & 6.0463859834 \\ \text { F38 } & -0.7375343565 & -0.0637350900 & 6.8201943995 \\ \text { F39 } & -2.8438457655 & 0.2159815460 & 6.3287608391 \\ \text { F40 } & -2.0094610773 & -1.7952274472 & 6.4362320471 \\ \text { C41 } & -3.8774267866 & 4.5016110437 & -2.2236856913 \\ \text { F42 } & -3.2023325595 & 5.2286598113 & -3.1401430314 \\ \text { F43 } & -4.9754065944 & 3.9944243098 & -2.8424221575 \\ \text { F44 } & -4.3187822238 & 5.3522707258 & -1.2685339119 \\ & & & \end{array}$

\section{$\mathrm{PPh}_{3}-\mathrm{CH}_{3}$}

$\mathrm{E}\left(\mathrm{B} 3 \mathrm{LYP}-\mathrm{D} 3 / 6-31+\mathrm{G}^{*}\right)($ benzene $)=-1076.07312834591$ $\mathrm{E}\left(\mathrm{M} 06-2 \mathrm{X} / 6-311+\mathrm{G}^{* *}\right)=-1075.86275194578$

$\mathrm{G}\left(\mathrm{M} 06-2 \mathrm{X} / 6-311+\mathrm{G}^{* *}\right)($ benzene $)=-1075.86275194578$

$\begin{array}{llll}\text { C1 } & -0.9369168261 & -0.2827695557 & 1.8616289007 \\ \text { C2 } & -1.7337947314 & -1.3315680787 & 2.3496900647 \\ \text { C3 } & -0.4061673241 & 0.6751137046 & 2.7461244136 \\ \text { C4 } & -1.9896128658 & -1.4242006989 & 3.7202715796 \\ \text { C5 } & -0.6675533897 & 0.5720280036 & 4.1128045902 \\ \text { C6 } & -1.4576285061 & -0.4767152529 & 4.5995370426 \\ \text { C7 } & -1.1197914032 & -1.6308857197 & -0.7722591925 \\ \text { C8 } & -2.2989397920 & -1.6673751352 & -1.5353877687 \\ \text { C9 } & -0.3281145164 & -2.7865636002 & -0.6359241215 \\ \text { C10 } & -2.6771522545 & -2.8553643030 & -2.1662214816\end{array}$




$\begin{array}{lrrr}\text { C11 } & -0.7153117662 & -3.9668602118 & -1.2714688089 \\ \text { C12 } & -1.8875620703 & -4.0016533878 & -2.0364674143 \\ \text { C13 } & -1.5894970857 & 1.2836853455 & -0.5591009736 \\ \text { C14 } & -2.6638624974 & 1.8049275295 & 0.1814480684 \\ \text { C15 } & -1.2826994717 & 1.8087796841 & -1.8281740053 \\ \text { C16 } & -3.4221320785 & 2.8532504171 & -0.3455593990 \\ \text { C17 } & -2.0458519953 & 2.8572167783 & -2.3433862282 \\ \text { C18 } & -3.1137381314 & 3.3791803091 & -1.6034914837 \\ \text { H19 } & -0.4625575306 & 1.4069846665 & -2.4187818463 \\ \text { H20 } & -1.8076576944 & 3.2658396174 & -3.3220232064 \\ \text { H21 } & -3.7048033843 & 4.1968938991 & -2.0087198531 \\ \text { H22 } & -4.2517337944 & 3.2578067985 & 0.2286562173 \\ \text { H23 } & -2.9089670162 & 1.4037847931 & 1.1609508586 \\ \text { H24 } & 0.1999643996 & 1.5010304772 & 2.3804649332 \\ \text { H25 } & -0.2562568200 & 1.3098053994 & 4.7969015882 \\ \text { H26 } & -1.6579227156 & -0.5540612898 & 5.6654373957 \\ \text { H27 } & -2.6040707968 & -2.2374625864 & 4.0979909990 \\ \text { H28 } & -2.1508535631 & -2.0725608872 & 1.6734477478 \\ \text { H29 } & 0.5801751554 & -2.7772007531 & -0.0375477222 \\ \text { H30 } & -0.1024717696 & -4.8587369763 & -1.1694472190 \\ \text { H31 } & -2.1839932494 & -4.9230984312 & -2.5316564927 \\ \text { H32 } & -3.5876040771 & -2.8821808606 & -2.7594969964 \\ \text { H33 } & -2.9173319376 & -0.7807293032 & -1.6439179237 \\ \text { P34 } & -0.6336711011 & -0.1120800959 & 0.0848559873 \\ \text { C35 } & 1.1448015577 & 0.1889965579 & -0.1930781478 \\ \text { H36 } & 1.3573287986 & 0.2021807585 & -1.2666064868 \\ \text { H37 } & 1.4300445313 & 1.1513051196 & 0.2431558389 \\ \text { H38 } & 1.7290292098 & -0.6071754285 & 0.2788263711\end{array}$

\section{$\mathrm{PPh}_{3}-\mathrm{BF}_{3}$}

$\mathrm{E}\left(\mathrm{B} 3 \mathrm{LYP}-\mathrm{D} 3 / 6-31+\mathrm{G}^{*}\right)($ benzene $)=-1360.95901594199$

$\mathrm{E}\left(\mathrm{M} 06-2 \mathrm{X} / 6-311+\mathrm{G}^{* *}\right)=-1360.74804925328$

$\mathrm{G}\left(\mathrm{M} 06-2 \mathrm{X} / 6-311+\mathrm{G}^{* *}\right)($ benzene $)=-1360.521110043$

$\begin{array}{lrrr}\text { C1 } & -1.3932207991 & -0.3836340512 & 1.6127549475 \\ \text { C2 } & -2.3977222333 & -1.1576968667 & 2.2149007029 \\ \text { C3 } & -0.5518893574 & 0.4137514728 & 2.4088677733 \\ \text { C4 } & -2.5631877453 & -1.1284185870 & 3.6027740777 \\ \text { C5 } & -0.7254763797 & 0.4392011314 & 3.7945087166 \\ \text { C6 } & -1.7298613151 & -0.3299885437 & 4.3927375788 \\ \text { C7 } & -2.0501723400 & 1.0569533420 & -0.8676967906 \\ \text { P8 } & -1.1407890497 & -0.3767496543 & -0.1964378336 \\ \text { H9 } & 0.2377065969 & 1.0039557244 & 1.9516737623 \\ \text { H10 } & -0.0696683197 & 1.0538124835 & 4.4063390768 \\ \text { H11 } & -1.8578787192 & -0.3126165650 & 5.4725802067 \\ \text { H12 } & -3.3402660580 & -1.7327551704 & 4.0649398694 \\ \text { H13 } & -3.0417042718 & -1.7894676662 & 1.6085294006 \\ \text { F14 } & 1.3424521305 & 0.9312869064 & -0.2290776012 \\ \text { B15 } & 0.8828161094 & -0.3015008252 & -0.6839910777\end{array}$




$\begin{array}{lrrr}\text { C16 } & -3.7788926911 & 2.7512581137 & -0.7124591212 \\ \text { H17 } & -4.5869726083 & 3.2209590179 & -0.1567557135 \\ \text { C18 } & -3.0970061893 & 1.6652000907 & -0.1568459511 \\ \text { H19 } & -3.3753019158 & 1.3006291637 & 0.8285392401 \\ \text { C20 } & -1.6870094783 & 1.5462987570 & -2.1347601638 \\ \text { H21 } & -0.8683767443 & 1.0903351369 & -2.6849464143 \\ \text { C22 } & -2.3744089180 & 2.6313782840 & -2.6833019182 \\ \text { H23 } & -2.0866884011 & 3.0095429244 & -3.6613464419 \\ \text { C24 } & -3.4196735828 & 3.2338732819 & -1.9750487457 \\ \text { H25 } & -3.9485924606 & 4.0818113113 & -2.4038256382 \\ \text { C26 } & -3.0898095058 & -4.2437290731 & -1.8248222089 \\ \text { C27 } & -3.7948999551 & -3.0387055658 & -1.9007976584 \\ \text { C28 } & -3.2220879092 & -1.8586855183 & -1.4172293855 \\ \text { C29 } & -1.9365106614 & -1.8833960023 & -0.8546055093 \\ \text { C30 } & -1.2258628960 & -3.0948469559 & -0.7850046945 \\ \text { C31 } & -1.8065781252 & -4.2696781818 & -1.2671677222 \\ \text { H32 } & -3.5361653683 & -5.1597621523 & -2.2049779616 \\ \text { H33 } & -4.7896276098 & -3.0136400435 & -2.3396043846 \\ \text { H34 } & -3.7724384175 & -0.9243317551 & -1.4880216961 \\ \text { H35 } & -0.2247143034 & -3.1202475811 & -0.3628492611 \\ \text { H36 } & -1.2522150955 & -5.2035942573 & -1.2145846777 \\ \text { F37 } & 1.4776763279 & -1.3720430037 & -0.0236659116 \\ \text { F38 } & 0.9519644342 & -0.4184345982 & -2.0687200902\end{array}$

$\mathrm{PPh}_{3}-\mathrm{BPh}_{3}$

$\mathrm{E}\left(\mathrm{B} 3 \mathrm{LYP}-\mathrm{D} 3 / 6-31+\mathrm{G}^{*}\right)($ benzene $)=-1756.26197242275$

$\mathrm{E}\left(\mathrm{M} 06-2 \mathrm{X} / 6-311+\mathrm{G}^{* *}\right)=-1755.88842507183$

$\mathrm{G}\left(\mathrm{M} 06-2 \mathrm{X} / 6-311+\mathrm{G}^{* *}\right)($ benzene $)=-1755.405508095$

$\begin{array}{lccr}\text { C1 } & -0.6826695944 & -0.6005387400 & 1.5903812077 \\ \text { C2 } & -1.1792738597 & -1.8568505927 & 1.9900637172 \\ \text { C3 } & -0.3065836942 & 0.2762677382 & 2.6340727836 \\ \text { C4 } & -1.2716828778 & -2.2296943530 & 3.3369696477 \\ \text { C5 } & -0.3837024976 & -0.0855181030 & 3.9812426272 \\ \text { C6 } & -0.8638178209 & -1.3494973115 & 4.3427621827 \\ \text { C7 } & -1.3087412296 & 1.2341466065 & -0.3490019809 \\ \text { C8 } & -2.1471996860 & 1.9007198007 & 0.5664002904 \\ \text { C9 } & -1.1637064298 & 1.8482565786 & -1.6144343870 \\ \text { C10 } & -2.7790534305 & 3.1121021475 & 0.2574824216 \\ \text { C11 } & -1.7802476376 & 3.0607039595 & -1.9335013644 \\ \text { C12 } & -2.5909032173 & 3.7066942207 & -0.9930146871 \\ \text { B13 } & -0.5717627403 & -0.1734010857 & 0.0208897712 \\ \text { P14 } & 1.5219846167 & 0.2602464953 & -0.2407849718 \\ \text { C15 } & 2.5672775529 & 1.2289856554 & -4.6724759653 \\ \text { C16 } & 2.9303694311 & 2.1263501021 & -3.6647398451 \\ \text { C17 } & 2.6403287586 & 1.8403120442 & -2.3271676434 \\ \text { C18 } & 1.9761533745 & 0.6526268702 & -1.9826322443 \\ \text { C19 } & 1.6198352743 & -0.2512505838 & -3.0022137259\end{array}$




\begin{tabular}{|c|c|c|c|}
\hline $\mathrm{C} 20$ & 1.9158320952 & 0.0373267205 & -4.3358526912 \\
\hline $\mathrm{H} 21$ & 2.7916154058 & 1.4550431892 & -5.7125849587 \\
\hline $\mathrm{H} 22$ & 3.4417292138 & 3.0530288177 & -3.9159529280 \\
\hline $\mathrm{H} 23$ & 2.9317641971 & 2.5492076927 & -1.5593508633 \\
\hline $\mathrm{H} 24$ & 1.1089693598 & -1.1764700631 & -2.7624043843 \\
\hline H25 & 1.6311455755 & -0.6702959236 & -5.1111393703 \\
\hline $\mathrm{C} 26$ & 2.8513801696 & 3.9180656219 & 2.3109828899 \\
\hline $\mathrm{C} 27$ & 1.7805781819 & 4.0219717594 & 1.4167320257 \\
\hline $\mathrm{C} 28$ & 1.4003069776 & 2.9253161582 & 0.6415027088 \\
\hline C29 & 2.0936072034 & 1.7044318204 & 0.7482877878 \\
\hline C 30 & 3.1744528023 & 1.6107785786 & 1.6389644713 \\
\hline C31 & 3.5476762335 & 2.7111217910 & 2.4169650959 \\
\hline H32 & 3.1406416426 & 4.7721155595 & 2.9193937099 \\
\hline H33 & 1.2326262781 & 4.9568433462 & 1.3237219838 \\
\hline H34 & 3.7270720844 & 0.6821888319 & 1.7362752016 \\
\hline H35 & 4.3842717295 & 2.6213630163 & 3.1064420938 \\
\hline C36 & 4.2807643703 & -3.2730551861 & 1.0206712504 \\
\hline C37 & 4.4517458906 & -2.6744541934 & -0.2308976255 \\
\hline C38 & 2.6389914915 & -1.1232940682 & 0.2437817781 \\
\hline H39 & 4.9126735641 & -4.1065861722 & 1.3190824690 \\
\hline $\mathrm{H} 40$ & 5.2189354233 & -3.0377808177 & -0.9112024508 \\
\hline H41 & -1.6302811798 & 3.4987115533 & -2.9189016299 \\
\hline H42 & -3.0743338423 & 4.6510371230 & -1.2355149970 \\
\hline H43 & -3.4187128430 & 3.5903360280 & 0.9975241317 \\
\hline H44 & -2.3159389911 & 1.4675589040 & 1.5478144534 \\
\hline H45 & 0.5619709374 & 3.0240068675 & -0.0380252259 \\
\hline H46 & -0.5522442328 & 1.3701582935 & -2.3735269183 \\
\hline H47 & 0.0566370415 & 1.2699272430 & 2.3919401401 \\
\hline H48 & -1.5048217190 & -2.5659783422 & 1.2350371757 \\
\hline H49 & -1.6634982221 & -3.2114776217 & 3.5980975890 \\
\hline $\mathrm{H} 50$ & -0.9280095086 & -1.6382066214 & 5.3900009145 \\
\hline H51 & -0.0737465085 & 0.6223271619 & 4.7480586539 \\
\hline C52 & -0.8498816326 & -1.3815237178 & -1.0394687739 \\
\hline C53 & -0.1570245257 & -2.6123247793 & -0.9727982115 \\
\hline H54 & 0.5697882662 & -2.7744007183 & -0.1829753590 \\
\hline C55 & 3.2939656209 & -2.7921271713 & 1.8881751883 \\
\hline H56 & 3.1530174218 & -3.2482433459 & 2.8652308551 \\
\hline C57 & 3.6385105642 & -1.6040732815 & -0.6175928615 \\
\hline H58 & 3.7871563610 & -1.1514549494 & -1.5925656171 \\
\hline C59 & 2.4771491723 & -1.7267303321 & 1.5057917792 \\
\hline $\mathrm{H} 60$ & 1.7147018404 & -1.3729435952 & 2.1897330807 \\
\hline C61 & -0.3658316493 & -3.6409838021 & -1.8952430125 \\
\hline H62 & 0.2014501851 & -4.5662493606 & -1.8086615769 \\
\hline C63 & -1.8008603276 & -1.2660587519 & -2.0725665866 \\
\hline H64 & -2.3822040711 & -0.3534872579 & -2.1626985735 \\
\hline C65 & -2.0271483777 & -2.2916699205 & -2.9988540612 \\
\hline H66 & -2.7714455832 & -2.1557442860 & -3.7818255693 \\
\hline C67 & -1.3017065547 & -3.4834565055 & -2.9240001730 \\
\hline H68 & -1.4678634328 & -4.2795608324 & -3.6471154998 \\
\hline
\end{tabular}




\section{$\mathrm{PPh}_{3}-\mathrm{B}\left(\mathrm{C}_{6} \mathrm{~F}_{5}\right)_{3}$}

$\mathrm{E}\left(\mathrm{B} 3 \mathrm{LYP}-\mathrm{D} 3 / 6-31+\mathrm{G}^{*}\right)$ (benzene $)=-3244.80995015310$

$\mathrm{E}\left(\mathrm{M} 06-2 \mathrm{X} / 6-311+\mathrm{G}^{* *}\right)=-3244.41970360054$

$\mathrm{G}\left(\mathrm{M} 06-2 \mathrm{X} / 6-311+\mathrm{G}^{* *}\right)($ benzene $)=-3244.076673$

\begin{tabular}{|c|c|c|c|}
\hline P1 & 10.6212966898 & 1.6375239883 & 3.5326137274 \\
\hline $\mathrm{C} 2$ & 11.0499542048 & 2.4691918697 & 5.1145081757 \\
\hline 3 & 12.0528778209 & 3.4488147188 & $.1721634 / 9$ \\
\hline & 12.3970524101 & 4.0319565151 & 6.393417485 \\
\hline 5 & 11.7437353651 & & 7.5669464097 \\
\hline 6 & 10.7329819728 & & 7.5144057198 \\
\hline & 10.3875505017 & 2.0975577244 & 463519 \\
\hline & & 2.2989588879 & \\
\hline 9 & 773 & & \\
\hline & & & \\
\hline & & & \\
\hline & & & \\
\hline & & & \\
\hline & & & \\
\hline & & & \\
\hline & & & \\
\hline & & & \\
\hline & & & \\
\hline & & & \\
\hline & & & \\
\hline & & & \\
\hline & & & \\
\hline & & & \\
\hline & & & 443 \\
\hline & & & \\
\hline & & & 902 \\
\hline & & & \\
\hline & & & 0781 \\
\hline & & & 10958 \\
\hline & & & \\
\hline & & & \\
\hline & & & \\
\hline & & & \\
\hline & & & \\
\hline & & 051 & \\
\hline & & & 7.60 \\
\hline & & & \\
\hline & & 2818 & \\
\hline & & & \\
\hline & & & \\
\hline 41 & 10.3070863916 & -1.6231278828 & 6.1332584899 \\
\hline 42 & 9.2134948006 & -2.3082165069 & 5.7324109988 \\
\hline $\mathrm{C} 43$ & 11.8385150717 & -1.0185145189 & 2.5821632654 \\
\hline & 13.0015044337 & -1.7411389159 & 2.877513928 \\
\hline
\end{tabular}




$\begin{array}{lccc}\text { F45 } & 13.1911244527 & -2.2992394813 & 4.0934385286 \\ \text { C46 } & 14.0347510256 & -1.9513485632 & 1.9637179011 \\ \text { F47 } & 15.1408053200 & -2.6213859067 & 2.3294091847 \\ \text { C48 } & 13.9229367698 & -1.4620954517 & 0.6671166399 \\ \text { F49 } & 14.9173921370 & -1.6301480794 & -0.2149116671 \\ \text { C50 } & 12.7605911683 & -0.7928456197 & 0.3004661726 \\ \text { F51 } & 12.6254445940 & -0.3077690734 & -0.9443373335 \\ \text { C52 } & 11.7641437905 & -0.6044521980 & 1.2472721734 \\ \text { F53 } & 10.6620418340 & 0.0597388478 & 0.8170362547 \\ \text { H54 } & 12.5769359144 & 3.7518950487 & 4.2724086656 \\ \text { H55 } & 13.1833661942 & 4.7813507294 & 6.4246422312 \\ \text { H56 } & 12.0235357736 & 4.0960789202 & 8.5174663200 \\ \text { H57 } & 10.2186938076 & 2.3805881755 & 8.4214524105 \\ \text { H58 } & 9.6133039613 & 1.3422655768 & 6.2793641210 \\ \text { H59 } & 8.7784917424 & 1.0376341359 & 1.3199226140 \\ \text { H60 } & 6.5375615326 & 1.8476879458 & 0.7215681318 \\ \text { H61 } & 5.4059482740 & 3.5618853559 & 2.1316475817 \\ \text { H62 } & 6.5739078947 & 4.4801178776 & 4.1291498908 \\ \text { H63 } & 8.8234497501 & 3.6808055012 & 4.7301712952 \\ \text { H64 } & 10.3041940680 & 3.5148042407 & 1.2480294180 \\ \text { H65 } & 11.8738038025 & 4.3453938882 & -0.4565077432 \\ \text { H66 } & 14.2600922966 & 3.6386762119 & -0.4193806835 \\ \text { H67 } & 15.0639857731 & 2.1063095120 & 1.3735931415 \\ \text { H68 } & 13.5022027612 & 1.2653152929 & 3.0728779936\end{array}$

\section{$\mathrm{PPh}_{3}-\mathrm{CPh}_{3}$}

$\mathrm{E}\left(\mathrm{B} 3 \mathrm{LYP}-\mathrm{D} 3 / 6-31+\mathrm{G}^{*}\right)($ benzene $)=-1769.27072684139$

$\mathrm{E}\left(\mathrm{M} 06-2 \mathrm{X} / 6-311+\mathrm{G}^{* *}\right)=-1768.896411484$

$\mathrm{G}\left(\mathrm{M} 06-2 \mathrm{X} / 6-311+\mathrm{G}^{* *}\right)($ benzene $)=-1768.432517021$

$\begin{array}{llcc}\text { C1 } & -0.5836967678 & -0.5756258093 & 1.4685614110 \\ \text { C2 } & -1.1883437872 & -1.7980644278 & 1.7983729360 \\ \text { C3 } & -0.1989190418 & 0.2671839879 & 2.5315405736 \\ \text { C4 } & -1.3575257024 & -2.1849209685 & 3.1329244510 \\ \text { C5 } & -0.3580701710 & -0.1197032074 & 3.8622534536 \\ \text { C6 } & -0.9303124360 & -1.3580130345 & 4.1724354317 \\ \text { C7 } & -1.1780381905 & 1.1689863756 & -0.3100179369 \\ \text { C8 } & -2.0974212496 & 1.7055722506 & 0.6051935618 \\ \text { C9 } & -1.0365812651 & 1.8262929242 & -1.5495056299 \\ \text { C10 } & -2.8147481576 & 2.8717747206 & 0.3129599393 \\ \text { C11 } & -1.7441415230 & 2.9927176384 & -1.8398006660 \\ \text { C12 } & -2.6334177122 & 3.5306014339 & -0.9036817458 \\ \text { P13 } & 1.5416168875 & 0.2703342974 & -0.2424176914 \\ \text { C14 } & 2.5412784236 & 1.1853304084 & -4.6788943972 \\ \text { C15 } & 2.8446093528 & 2.1149561124 & -3.6808852748 \\ \text { C16 } & 2.5464667041 & 1.8383939304 & -2.3442984384 \\ \text { C17 } & 1.9341281243 & 0.6212957856 & -1.9947340875\end{array}$




\begin{tabular}{|c|c|c|c|}
\hline $\mathrm{C} 18$ & 1.6475953312 & -0.3212140309 & -3.0035015246 \\
\hline C19 & 1.9487015049 & -0.0344734545 & -4.3350268808 \\
\hline $\mathrm{H} 20$ & 2.7719101507 & 1.4060329851 & -5.7183907708 \\
\hline $\mathrm{H} 21$ & 3.3164245884 & 3.0600984277 & -3.9382413616 \\
\hline $\mathrm{H} 22$ & 2.7966323884 & 2.5734146916 & -1.5878927395 \\
\hline $\mathrm{H} 23$ & 1.1977732711 & -1.2754368494 & -2.7624944903 \\
\hline $\mathrm{H} 24$ & 1.7203971552 & -0.7691895638 & -5.1031271838 \\
\hline $\mathrm{C} 25$ & 2.8429267697 & 3.9436974871 & 2.2719722816 \\
\hline $\mathrm{C} 26$ & 1.7937089381 & 4.0633674185 & 1.3545864385 \\
\hline $\mathrm{C} 27$ & 1.3972024363 & 2.9669938844 & 0.5892369130 \\
\hline $\mathrm{C} 28$ & 2.0501043034 & 1.7267308921 & 0.7404180579 \\
\hline C29 & 3.1159257207 & 1.6163775796 & 1.6516877221 \\
\hline $\mathrm{C} 30$ & 3.5055027365 & 2.7217609133 & 2.4123124774 \\
\hline H31 & 3.1456058992 & 4.8007383221 & 2.8687595027 \\
\hline H32 & 1.2797411518 & 5.0133014417 & 1.2304615773 \\
\hline H33 & 3.6477041750 & 0.6799042655 & 1.7752672490 \\
\hline H34 & 4.3294775221 & 2.6229824279 & 3.1147383441 \\
\hline C35 & 4.2158240549 & -3.2885151372 & 1.0679972137 \\
\hline C36 & 4.3400763014 & -2.7448099327 & -0.213092985 \\
\hline C37 & 2.5863850558 & -1.1376493470 & 0.283226698 \\
\hline H38 & 4.8427077085 & -4.1241433376 & 1.369759785 \\
\hline H39 & 5.0669933728 & -3.1509669229 & -0.912222557 \\
\hline $\mathrm{H} 40$ & -1.6060447673 & 3.4735949177 & -2.805392062 \\
\hline H41 & -3.1873426699 & 4.4389367671 & -1.1284636196 \\
\hline $\mathrm{H} 42$ & -3.5204535142 & 3.2581616180 & 1.0446459593 \\
\hline $\mathrm{H} 43$ & -2.2788195528 & 1.2169335364 & 1.5541821612 \\
\hline H44 & 0.5865958628 & 3.0870508334 & -0.1176281354 \\
\hline H45 & -0.3852893894 & 1.4202095429 & -2.3121581206 \\
\hline H46 & 0.2113864835 & 1.2476566971 & 2.3295007648 \\
\hline $\mathrm{H} 47$ & -1.5473597645 & -2.4620928087 & 1.022560398 \\
\hline $\mathrm{H} 48$ & -1.8306010761 & -3.1392825085 & 3.35227618 \\
\hline H49 & -1.0567234797 & -1.6635283044 & 5.208326322 \\
\hline H50 & -0.0443132632 & 0.5550565715 & 4.655184063 \\
\hline C51 & -0.7358860531 & -1.2635439121 & -1.001178160 \\
\hline C52 & -0.0556793316 & -2.4977716190 & -0.9506521596 \\
\hline H53 & 0.6931351529 & -2.6781535744 & -0.1908401557 \\
\hline C54 & 3.2879311289 & -2.7468979023 & 1.9643902954 \\
\hline H55 & 3.1917870552 & -3.1546063212 & 2.9675922374 \\
\hline C56 & 3.5334009016 & -1.6741092732 & -0.6075729385 \\
\hline H57 & 3.6527730915 & -1.2667611390 & -1.6050931769 \\
\hline C58 & 2.4792356485 & -1.6766360651 & 1.581687858 \\
\hline H59 & 1.7765949946 & -1.2702137575 & 2.297085325 \\
\hline C60 & -0.3390182223 & -3.5226800533 & -1.85287773 \\
\hline H61 & 0.2101658610 & -4.4588961534 & -1.786619452 \\
\hline C62 & -1.7506872387 & -1.1233019044 & -1.9606957008 \\
\hline H63 & -2.3376855557 & -0.2153695734 & -2.0142366111 \\
\hline C64 & -2.0407520946 & -2.1528828281 & -2.8635550568 \\
\hline H65 & -2.8323183442 & -2.0095782597 & -3.5954604980 \\
\hline C66 & -1.3281934759 & -3.3516469270 & -2.8265845843 \\
\hline H67 & -1.5500669071 & -4.1480895432 & -3.5329438560 \\
\hline C68 & -0.3959904766 & -0.1310093918 & -0.0018728730 \\
\hline
\end{tabular}




\section{P-triptycene}

$\mathrm{E}\left(\mathrm{B} 3 \mathrm{LYP}-\mathrm{D} 3 / 6-31+\mathrm{G}^{*}\right)($ benzene $)=-1073.27574221013$

$\mathrm{E}\left(\mathrm{M} 06-2 \mathrm{X} / 6-311+\mathrm{G}^{* *}\right)=-1073.08558650274$

$\mathrm{G}\left(\mathrm{M} 06-2 \mathrm{X} / 6-311+\mathrm{G}^{* *}\right)($ benzene $)=-1072.871165742$

$\begin{array}{lccc}\text { C1 } & -2.1014960165 & 1.3727823653 & 6.3218563477 \\ \text { C2 } & -2.1940848368 & 2.6473870721 & 5.7436779049 \\ \text { C3 } & -1.0805530586 & 3.4913556041 & 5.7162423791 \\ \text { C4 } & 0.1359978945 & 3.0648162897 & 6.2682402289 \\ \text { C5 } & 0.2300057960 & 1.7956841740 & 6.8446000130 \\ \text { C6 } & 1.6729841659 & -0.5195350769 & 6.7232813753 \\ \text { C7 } & 2.7432194620 & -1.1182076033 & 6.0538993990 \\ \text { C8 } & 2.5639388076 & -2.3555764656 & 5.4186601483 \\ \text { C9 } & 1.3170834205 & -2.9857071247 & 5.4560735779 \\ \text { C10 } & 0.2414980430 & -2.3857872201 & 6.1274956537 \\ \text { C11 } & 0.4166046494 & -1.1556894808 & 6.7604966328 \\ \text { C12 } & -0.8927887425 & 0.9451382984 & 6.8705642914 \\ \text { H13 } & -2.9694395691 & 0.7158564158 & 6.3386956018 \\ \text { H14 } & -3.1374768772 & 2.9778196441 & 5.3145640227 \\ \text { H15 } & -1.1546841201 & 4.4785708406 & 5.2652347302 \\ \text { H16 } & 1.0037979053 & 3.7215911630 & 6.2458819095 \\ \text { H17 } & 3.7142750683 & -0.6271907131 & 6.0248533666 \\ \text { H18 } & 3.3964337103 & -2.8229607441 & 4.8971124979 \\ \text { H19 } & 1.1774520674 & -3.9453004906 & 4.9631976505 \\ \text { H20 } & -0.7286566272 & -2.8791231467 & 6.1546798417 \\ \text { C21 } & -0.4357336153 & -0.4856862639 & 11.3580553928 \\ \text { C22 } & 0.7442787537 & 0.2398425619 & 11.5430313985 \\ \text { C23 } & 1.4403827196 & 0.7405464115 & 10.4334399857 \\ \text { C24 } & 0.9503489500 & 0.5118963030 & 9.1454474554 \\ \text { C25 } & -0.2386557927 & -0.2206712113 & 8.9596472895 \\ \text { C26 } & -0.9290418228 & -0.7163934254 & 10.0653831447 \\ \text { H27 } & -0.9752131332 & -0.8751256077 & 12.2186321326 \\ \text { H28 } & 1.1259035406 & 0.4152431417 & 12.5465624890 \\ \text { H29 } & 2.3600788794 & 1.3049293482 & 10.5769566025 \\ \text { H30 } & -1.8463707139 & -1.2852977595 & 9.9240907736 \\ \text { P31 } & 1.7933555868 & 1.1203958644 & 7.6001225187 \\ \text { C32 } & -0.6968754218 & -0.4282533039 & 7.5148891041 \\ \text { H33 } & -1.6257914919 & -1.0058213187 & 7.4834552587\end{array}$

P-triptycene $+\mathbf{H}^{+}$

$\mathrm{E}\left(\mathrm{B} 3 \mathrm{LYP}-\mathrm{D} 3 / 6-31+\mathrm{G}^{*}\right)($ benzene $)=-1073.62956758328$

$\begin{array}{llll}\text { C1 } & -2.1116976887 & 1.3720543725 & 6.3063708589 \\ \text { C2 } & -2.2002311314 & 2.6489661434 & 5.7332753861 \\ \text { C3 } & -1.0951985723 & 3.5054768257 & 5.7125062283 \\ \text { C4 } & 0.1244742382 & 3.0967774611 & 6.2663291421 \\ \text { C5 } & 0.1993014576 & 1.8239330591 & 6.8338615876 \\ \text { C6 } & 1.6855470212 & -0.5547046736 & 6.7040257890 \\ \text { C7 } & 2.7684413243 & -1.1355669860 & 6.0426373301\end{array}$




$\begin{array}{lccc}\text { C8 } & 2.5741702253 & -2.3684528402 & 5.4070625793 \\ \text { C9 } & 1.3219926219 & -2.9896108096 & 5.4426538681 \\ \text { C10 } & 0.2420344743 & -2.3959713087 & 6.1120584315 \\ \text { C11 } & 0.4152832108 & -1.1705497272 & 6.7501675207 \\ \text { C12 } & -0.9080354238 & 0.9475567199 & 6.8633427584 \\ \text { H13 } & -2.9784506618 & 0.7148553323 & 6.3157912634 \\ \text { H14 } & -3.1420316510 & 2.9749095680 & 5.2983853070 \\ \text { H15 } & -1.1792878336 & 4.4919540100 & 5.2641355820 \\ \text { H16 } & 0.9843313381 & 3.7623433890 & 6.2536050367 \\ \text { H17 } & 3.7419575813 & -0.6517409493 & 6.0174006647 \\ \text { H18 } & 3.4017613607 & -2.8413165398 & 4.8848320443 \\ \text { H19 } & 1.1812391251 & -3.9462791007 & 4.9453395800 \\ \text { H20 } & -0.7271838440 & -2.8893404592 & 6.1329612834 \\ \text { C21 } & -0.4666575009 & -0.5013935935 & 11.3632444329 \\ \text { C22 } & 0.7110392409 & 0.2236240286 & 11.5696761130 \\ \text { C23 } & 1.4263326519 & 0.7323376193 & 10.4785244515 \\ \text { C24 } & 0.9324103499 & 0.4954822012 & 9.1952937035 \\ \text { C25 } & -0.2555577841 & -0.2348382467 & 8.9700376061 \\ \text { C26 } & -0.9526685984 & -0.7325002953 & 10.0682435597 \\ \text { H27 } & -1.0148061321 & -0.8925882848 & 12.2168957026 \\ \text { H28 } & 1.0757439207 & 0.3947112410 & 12.5790548060 \\ \text { H29 } & 2.3424336375 & 1.2967216176 & 10.6355998365 \\ \text { H30 } & -1.8699866945 & -1.2981915746 & 9.9208484320 \\ \text { C31 } & -0.6998836538 & -0.4324489606 & 7.5108430534 \\ \text { H32 } & -1.6267085228 & -1.0096421637 & 7.4748474112 \\ \text { P33 } & 1.6155224860 & 1.0097881522 & 7.6045349025 \\ \text { H34 } & 2.8085274648 & 1.7521571415 & 7.6523185896\end{array}$

P-triptycene- $\mathrm{CH}_{3}$

E(B3LYP-D3/6-31+G*)(benzene) $=-1113.01234036558$

$\mathrm{E}\left(\mathrm{M} 06-2 \mathrm{X} / 6-311+\mathrm{G}^{* *}\right)=-1112.76560300029$

$\mathrm{G}\left(\mathrm{M} 06-2 \mathrm{X} / 6-311+\mathrm{G}^{* *}\right)($ benzene $)=-1112.548744961$

$\begin{array}{lccc}\text { C1 } & -2.1358766242 & 1.3608186379 & 6.3181989885 \\ \text { C2 } & -2.2249008894 & 2.6372294827 & 5.7442599782 \\ \text { C3 } & -1.1162346608 & 3.4882574273 & 5.7119456861 \\ \text { C4 } & 0.1054732616 & 3.0733187703 & 6.2565572767 \\ \text { C5 } & 0.1829601586 & 1.8014105214 & 6.8248294275 \\ \text { C6 } & 1.6603022458 & -0.5673753358 & 6.7027738911 \\ \text { C7 } & 2.7390109174 & -1.1497328010 & 6.0360934359 \\ \text { C8 } & 2.5459021398 & -2.3845741016 & 5.4041871815 \\ \text { C9 } & 1.2952074972 & -3.0077425798 & 5.4471122081 \\ \text { C10 } & 0.2175120982 & -2.4128997161 & 6.1190111917 \\ \text { C11 } & 0.3922473393 & -1.1854550279 & 6.7533430569 \\ \text { C12 } & -0.9284200586 & 0.9321992711 & 6.8637653389 \\ \text { H13 } & -3.0056910368 & 0.7077907776 & 6.3369425725 \\ \text { H14 } & -3.1697305786 & 2.9670669786 & 5.3191742717\end{array}$




$\begin{array}{lrrr}\text { H15 } & -1.1993837323 & 4.4748276673 & 5.2635336829 \\ \text { H16 } & 0.9682020213 & 3.7347263965 & 6.2318569766 \\ \text { H17 } & 3.7117664887 & -0.6649208519 & 6.0026606813 \\ \text { H18 } & 3.3723779450 & -2.8567035553 & 4.8794136330 \\ \text { H19 } & 1.1537520398 & -3.9660906201 & 4.9533755890 \\ \text { H20 } & -0.7511600301 & -2.9072468014 & 6.1444894546 \\ \text { C21 } & -0.4654874972 & -0.4964103470 & 11.3653964436 \\ \text { C22 } & 0.7138318050 & 0.2287192267 & 11.5600474085 \\ \text { C23 } & 1.4206193934 & 0.7309781777 & 10.4603232007 \\ \text { C24 } & 0.9191716833 & 0.4898786146 & 9.1805520449 \\ \text { C25 } & -0.2697106927 & -0.2412426046 & 8.9697666626 \\ \text { C26 } & -0.9599769738 & -0.7334185452 & 10.0746543691 \\ \text { H27 } & -1.0080454041 & -0.8832449201 & 12.2245796925 \\ \text { H28 } & 1.0860675448 & 0.4044995919 & 12.5659609773 \\ \text { H29 } & 2.3376325435 & 1.2954378670 & 10.6114028245 \\ \text { H30 } & -1.8783758314 & -1.2997179440 & 9.9364373770 \\ \text { C31 } & -0.7202900632 & -0.4453121377 & 7.5141275475 \\ \text { H32 } & -1.6479485626 & -1.0217433827 & 7.4825110325 \\ \text { P33 } & 1.6153262167 & 1.0071604294 & 7.5931655424 \\ \text { C34 } & 3.1486395808 & 1.9613220926 & 7.6461803198 \\ \text { H35 } & 3.9317016455 & 1.3710862130 & 8.1343148558 \\ \text { H36 } & 3.4627693866 & 2.2082300540 & 6.6262832717 \\ \text { H37 } & 2.9859669960 & 2.8860226620 & 8.2106017582\end{array}$

\section{P-triptycene- $\mathrm{BF}_{3}$}

$\mathrm{E}\left(\mathrm{B} 3 \mathrm{LYP}-\mathrm{D} 3 / 6-31+\mathrm{G}^{*}\right)($ benzene $)=-1397.87653287699$

$\mathrm{E}\left(\mathrm{M} 06-2 \mathrm{X}-\mathrm{D} 3 / 6-311+\mathrm{G}^{* *}\right)=-1397.65906974397$

$\mathrm{G}\left(\mathrm{M} 06-2 \mathrm{X} / 6-311+\mathrm{G}^{* *}\right)($ benzene $)=-1397.441648155$

$\begin{array}{lrrl}\text { C1 } & -2.2297944505 & 1.3033343241 & 6.3181442071 \\ \text { C2 } & -2.3158831169 & 2.5832030009 & 5.7504687506 \\ \text { C3 } & -1.2030151822 & 3.4282511160 & 5.7239230371 \\ \text { C4 } & 0.0161129680 & 3.0026085827 & 6.2671635592 \\ \text { C5 } & 0.0935937597 & 1.7285430087 & 6.8299734165 \\ \text { C6 } & 1.5560430309 & -0.6167250434 & 6.7072286421 \\ \text { C7 } & 2.6376374270 & -1.2006663920 & 6.0472941899 \\ \text { C8 } & 2.4555318828 & -2.4382340166 & 5.4161744711 \\ \text { C9 } & 1.2084444363 & -3.0681603895 & 5.4537020338 \\ \text { C10 } & 0.1262518298 & -2.4748071663 & 6.1199981755 \\ \text { C11 } & 0.2949994237 & -1.2439127502 & 6.7510552709 \\ \text { C12 } & -1.0224062373 & 0.8689109681 & 6.8618598814 \\ \text { H13 } & -3.1015980771 & 0.6524228782 & 6.3349001827 \\ \text { H14 } & -3.2594949081 & 2.9196993808 & 5.3272584206 \\ \text { H15 } & -1.2799101700 & 4.4186090862 & 5.2817285689 \\ \text { H16 } & 0.8857287616 & 3.6547044252 & 6.2497215057 \\ \text { H17 } & 3.6051324763 & -0.7054952213 & 6.0225836223 \\ \text { H18 } & 3.2883711939 & -2.9065280964 & 4.8973068971 \\ \text { H19 } & 1.0735088018 & -4.0286553073 & 4.9618552596 \\ \text { H20 } & -0.8410282722 & -2.9726116457 & 6.1443162004\end{array}$




$\begin{array}{lccc}\text { C21 } & -0.5605557848 & -0.5498087425 & 11.3574409792 \\ \text { C22 } & 0.6187393562 & 0.1767777512 & 11.5440606675 \\ \text { C23 } & 1.3210264323 & 0.6736086506 & 10.4382360658 \\ \text { C24 } & 0.8213477985 & 0.4291610680 & 9.1590963013 \\ \text { C25 } & -0.3666473142 & -0.3020724489 & 8.9602860585 \\ \text { C26 } & -1.0561964956 & -0.7913399225 & 10.0679180359 \\ \text { H27 } & -1.1011829111 & -0.9331366836 & 12.2197433364 \\ \text { H28 } & 0.9948985946 & 0.3585316078 & 12.5478682670 \\ \text { H29 } & 2.2386702291 & 1.2398429253 & 10.5761149481 \\ \text { H30 } & -1.9750553064 & -1.3583096331 & 9.9325505396 \\ \text { C31 } & -0.8195163058 & -0.5085591823 & 7.5081786560 \\ \text { H32 } & -1.7474233737 & -1.0858056464 & 7.4769635897 \\ \text { P33 } & 1.5771730295 & 0.9821840219 & 7.5902513663 \\ \text { B34 } & 3.3514025365 & 2.0877529331 & 7.6467814217 \\ \text { F35 } & 4.2840228398 & 1.2486698335 & 8.2352973283 \\ \text { F36 } & 3.6345629319 & 2.3840391051 & 6.3225866233 \\ \text { F37 } & 3.0570322461 & 3.2063245563 & 8.4107112275\end{array}$

\section{P-triptycene-BPh 3}

$\mathrm{E}\left(\mathrm{B} 3 \mathrm{LYP}-\mathrm{D} 3 / 6-31+\mathrm{G}^{*}\right)($ benzene $)=-1793.17244830619$

$\mathrm{E}\left(\mathrm{M} 06-2 \mathrm{X} / 6-311+\mathrm{G}^{* *}\right)=-1792.804253687$

$\mathrm{G}\left(\mathrm{M} 06-2 \mathrm{X} / 6-311+\mathrm{G}^{* *}\right)($ benzene $)=-1792.331887774$

$\begin{array}{lccc}\text { C1 } & 3.3923823880 & 0.1654182427 & -1.8833250757 \\ \text { C2 } & 2.0153976058 & -0.1320013033 & -1.8323079054 \\ \text { C3 } & 3.3174060406 & 2.0097080613 & -0.2191458480 \\ \text { C4 } & 1.3609726293 & -0.6528262044 & -2.9514618213 \\ \text { C5 } & 1.9293345057 & 1.9305949614 & 0.0141185798 \\ \text { C6 } & 3.9706593207 & 3.2347201862 & -0.0905250141 \\ \text { C7 } & 3.2446854841 & 4.3790851389 & 0.2666674961 \\ \text { C8 } & 1.8677700973 & 4.2983636226 & 0.4900328027 \\ \text { C9 } & 3.8357879951 & -0.3065869696 & 0.5125799506 \\ \text { C10 } & 2.5083632480 & -0.6468537191 & 0.8423700323 \\ \text { C11 } & 2.0843369812 & -0.8688652464 & -4.1312393390 \\ \text { C12 } & 3.4481051090 & -0.5715752944 & -4.1863743240 \\ \text { C13 } & 1.2024902842 & 3.0725180814 & 0.3617153004 \\ \text { C14 } & 4.1047286522 & -0.0552849681 & -3.0612340133 \\ \text { C15 } & 2.2440103098 & -1.5646824279 & 1.8616363884 \\ \text { C16 } & 3.3120858352 & -2.1566964184 & 2.5473343924 \\ \text { C17 } & 4.6290787105 & -1.8268091247 & 2.2189004462 \\ \text { C18 } & 4.8928190519 & -0.8990200304 & 1.2021353950 \\ \text { H19 } & 0.3070799274 & -0.9043788414 & -2.9092979489 \\ \text { H20 } & 5.0425860417 & 3.2993012208 & -0.2673946241 \\ \text { H21 } & 3.7575224148 & 5.3329041538 & 0.3671776485 \\ \text { H22 } & 4.0057735012 & -0.7435027898 & -5.1041976888 \\ \text { H23 } & 0.1307108480 & 3.0199599214 & 0.5175233416 \\ \text { H24 } & 5.1680356840 & 0.1725484675 & -3.1037491149 \\ \text { H25 } & 1.2235955175 & -1.8097095934 & 2.1339894995 \\ \text { H26 } & 5.4551318951 & -2.2888334376 & 2.7547217770\end{array}$




$\begin{array}{lrrr}\text { H27 } & 5.9191717295 & -0.6404673608 & 0.9490981573 \\ \text { P28 } & 1.2508923930 & 0.2256326598 & -0.1895603347 \\ \text { C29 } & 4.0345832501 & 0.7155500742 & -0.6086603420 \\ \text { H30 } & 5.0996105980 & 0.9031143863 & -0.7693864258 \\ \text { C31 } & -0.9228649381 & -0.3904356621 & 1.7246135205 \\ \text { C32 } & -1.5589022433 & -1.5355830711 & 2.2402177568 \\ \text { C33 } & -0.4398104768 & 0.5381894392 & 2.6709465152 \\ \text { C34 } & -1.6957507431 & -1.7508990172 & 3.6176870593 \\ \text { C35 } & -0.5606750944 & 0.3326719080 & 4.0472994902 \\ \text { C36 } & -1.1907882443 & -0.8203002022 & 4.5300215846 \\ \text { C37 } & -1.1134358902 & -1.4464672738 & -0.7891247871 \\ \text { C38 } & -2.1565670784 & -1.4527956694 & -1.7348542901 \\ \text { C39 } & -0.3760357995 & -2.6424521144 & -0.6613375186 \\ \text { C40 } & -2.4388304713 & -2.5774090206 & -2.5204990220 \\ \text { C41 } & -0.6408912024 & -3.7689844835 & -1.4432729790 \\ \text { C42 } & -1.6765503403 & -3.7406992397 & -2.3840605761 \\ \text { C43 } & -1.5280007594 & 1.2244482709 & -0.3911453404 \\ \text { C44 } & -2.4481805562 & 1.9161005160 & 0.4199344936 \\ \text { C45 } & -1.2985639277 & 1.7598831072 & -1.6762643622 \\ \text { C46 } & -3.0904823081 & 3.0824249357 & -0.0150793677 \\ \text { C47 } & -1.9242359959 & 2.9270440374 & -2.1201864970 \\ \text { C48 } & -2.8253231552 & 3.5990489128 & -1.2864233255 \\ \text { B49 } & -0.7979051388 & -0.1366465403 & 0.1222705665 \\ \text { H50 } & 3.1087734157 & -2.8719854824 & 3.3407132870 \\ \text { H51 } & 1.5778046093 & -1.2754934247 & -5.0032829002 \\ \text { H52 } & 1.3042628669 & 5.1880903967 & 0.7603423286 \\ \text { H53 } & -3.3155969453 & 4.5094500835 & -1.6253587162 \\ \text { H54 } & -1.7078568663 & 3.3123940890 & -3.1150787660 \\ \text { H55 } & -3.7964143834 & 3.5875999740 & 0.6420030507 \\ \text { H56 } & -2.6667768053 & 1.5403150812 & 1.4159332324 \\ \text { H57 } & -0.6077204166 & 1.2582259941 & -2.3511042466 \\ \text { H58 } & 0.0483450466 & 1.4483044010 & 2.3288351957 \\ \text { H59 } & -2.7607923752 & -0.5593522120 & -1.8667220873 \\ \text { H60 } & 0.4329661794 & -2.6988161257 & 0.0638259632 \\ \text { H61 } & -3.2544843872 & -2.5418216820 & -3.2408378723 \\ \text { H62 } & -1.8878266979 & -4.6138481639 & -2.9980295135 \\ \text { H63 } & -0.0389986054 & -4.6675209177 & -1.3195412165 \\ \text { H64 } & -1.9561523873 & -2.2781241492 & 1.5528487160 \\ \text { H65 } & -2.1973382200 & -2.6482306828 & 3.9763489697 \\ \text { H66 } & -1.2899729194 & -0.9878052793 & 5.6005578958 \\ \text { H67 } & -0.1639548381 & 1.0710782199 & 4.7417519325 \\ & & & \end{array}$

\section{P-triptycene- $\mathrm{B}\left(\mathrm{C}_{6} \mathrm{~F}_{5}\right)_{3}$}

$\mathrm{E}\left(\mathrm{B} 3 \mathrm{LYP}-\mathrm{D} 3 / 6-31+\mathrm{G}^{*}\right)($ benzene $)=-3281.71872396809$

$\mathrm{E}\left(\mathrm{M} 06-2 \mathrm{X} / 6-311+\mathrm{G}^{* *}\right)=-3281.33705412171$

$\mathrm{G}\left(\mathrm{M} 06-2 \mathrm{X} / 6-311+\mathrm{G}^{* *}\right)($ benzene $)=-3281.006770229$

$\begin{array}{llll}\mathrm{C} 1 & 3.4530348617 & 0.0674732246 & -1.8462090021\end{array}$ 


\begin{tabular}{|c|c|c|c|}
\hline $\mathrm{C} 2$ & 2.0763905554 & -0.2332026421 & -1.8088762305 \\
\hline C3 & 3.3232738473 & 2.0384553566 & -0.3398079516 \\
\hline $\mathrm{C} 4$ & 1.4530756667 & -0.8023055200 & -2.9224424690 \\
\hline $\mathrm{C} 5$ & 1.9364930654 & 1.9643359216 & -0.0990962987 \\
\hline C6 & 3.9683481276 & 3.2741518324 & -0.3001545228 \\
\hline C7 & 3.2401403219 & 4.4358731068 & -0.0175310688 \\
\hline $\mathrm{C} 8$ & 1.8693321115 & 4.3583663840 & 0.2380918664 \\
\hline C9 & 3.8627653967 & -0.2021411240 & 0.5850852177 \\
\hline $\mathrm{C} 10$ & 2.5398964059 & -0.5589071420 & 0.9161324304 \\
\hline C11 & 2.2068502786 & -1.0950546284 & -4.0643913869 \\
\hline $\mathrm{C} 12$ & 3.5740456387 & -0.8122681814 & -4.0974699612 \\
\hline $\mathrm{C} 13$ & 1.2144153856 & 3.1224057810 & 0.2027208522 \\
\hline C14 & 4.1962088328 & -0.2243985508 & -2.9895634350 \\
\hline C15 & 2.2950042087 & -1.4440793813 & 1.9691824764 \\
\hline C16 & 3.3673878443 & -1.9549801795 & 2.7084379456 \\
\hline $\mathrm{C} 17$ & 4.6776188857 & -1.5906814098 & 2.3914442611 \\
\hline C18 & 4.9259741983 & -0.7183614285 & 1.3250137780 \\
\hline H19 & 0.3898751821 & -1.0005827925 & -2.9222369735 \\
\hline $\mathrm{H} 20$ & 5.0390985829 & 3.3306196751 & -0.4849839084 \\
\hline $\mathrm{H} 21$ & 3.7466047368 & 5.3976225710 & 0.0117927860 \\
\hline $\mathrm{H} 22$ & 4.1561760682 & -1.0406500539 & -4.9870466531 \\
\hline $\mathrm{H} 23$ & 0.1585808060 & 3.0741023717 & 0.4324958921 \\
\hline $\mathrm{H} 24$ & 5.2585057281 & 0.0089769943 & -3.0177595806 \\
\hline H25 & 1.2878569577 & -1.7583162225 & 2.2071507181 \\
\hline H26 & 5.5086116179 & -1.9915084713 & 2.9669328106 \\
\hline $\mathrm{H} 27$ & 5.9470188365 & -0.4447916778 & 1.0674614150 \\
\hline P28 & 1.2975070834 & 0.2333637045 & -0.1989763495 \\
\hline $\mathrm{C} 29$ & 4.0620134396 & 0.7282922543 & -0.6104523324 \\
\hline $\mathrm{H} 30$ & 5.1263006511 & 0.9192650399 & -0.7687498086 \\
\hline C31 & -0.9333764994 & -0.5102822052 & 1.6997667511 \\
\hline C32 & -1.6385412466 & -1.6144193993 & 2.1903620263 \\
\hline C33 & -0.4070861341 & 0.3112905323 & 2.7014619426 \\
\hline C34 & -1.7770003941 & -1.9006908281 & 3.5487790828 \\
\hline C35 & -0.5195779551 & 0.0700688250 & 4.0640140176 \\
\hline C36 & -1.2107102325 & -1.0575852887 & 4.4968036454 \\
\hline C37 & -1.1642874422 & -1.3820457727 & -0.8786398157 \\
\hline C38 & -2.2648488302 & -1.4037883267 & -1.7419175787 \\
\hline C39 & -0.4296202198 & -2.5716805000 & -0.8725506753 \\
\hline $\mathrm{C} 40$ & -2.5861335804 & -2.4909155662 & -2.5551424415 \\
\hline $\mathrm{C} 41$ & -0.7102136836 & -3.6794691861 & -1.6608437872 \\
\hline $\mathrm{C} 42$ & -1.8028261186 & -3.6380761050 & -2.5215572103 \\
\hline $\mathrm{C} 43$ & -1.5384024782 & 1.2627830707 & -0.2922128448 \\
\hline C44 & -2.4799019737 & 1.9202845475 & 0.5070134851 \\
\hline $\mathrm{C} 45$ & -1.3220941527 & 1.8783412784 & -1.5291824016 \\
\hline $\mathrm{C} 46$ & -3.1166883190 & 3.1042336690 & 0.1334862831 \\
\hline $\mathrm{C} 47$ & -1.9325972687 & 3.0532262443 & -1.9465358227 \\
\hline $\mathrm{C} 48$ & -2.8402026103 & 3.6818936159 & -1.0994494560 \\
\hline B49 & -0.7745861108 & -0.1327154569 & 0.1103804658 \\
\hline F50 & -0.4566205692 & 1.3104822671 & -2.4044333454 \\
\hline F51 & -1.6546320651 & 3.5847763132 & -3.1487250261 \\
\hline F52 & -3.4413611786 & 4.8207721554 & -1.4693368202 \\
\hline
\end{tabular}




$\begin{array}{lrrr}\text { F53 } & -4.0045146037 & 3.6861033688 & 0.9576705874 \\ \text { F54 } & -2.8574455874 & 1.4255273630 & 1.7079467783 \\ \text { F55 } & 0.2659127466 & 1.4334150458 & 2.3469289776 \\ \text { F56 } & 0.0298527210 & 0.9076772520 & 4.9594802097 \\ \text { F57 } & -1.3332349651 & -1.3218862333 & 5.8046766919 \\ \text { F58 } & -2.4648176323 & -2.9844067832 & 3.9472415144 \\ \text { F59 } & -2.2675376409 & -2.4770538871 & 1.3597122850 \\ \text { F60 } & 0.6391488900 & -2.6814934634 & -0.0457709277 \\ \text { F61 } & 0.0579691855 & -4.7801894110 & -1.6004467019 \\ \text { F62 } & -2.0979820441 & -4.6884491877 & -3.2993661954 \\ \text { F63 } & -3.6571888399 & -2.4391141498 & -3.3653229708 \\ \text { F64 } & -3.1205521826 & -0.3592766243 & -1.8224574751 \\ \text { H65 } & 3.1737645486 & -2.6450048735 & 3.5261767947 \\ \text { H66 } & 1.7185142358 & -1.5373216063 & -4.9293900828 \\ \text { H67 } & 1.3049677097 & 5.2570626796 & 0.4746459896\end{array}$

\section{P-triptycene-CPh}

$\mathrm{E}\left(\mathrm{B} 3 \mathrm{LYP}-\mathrm{D} 3 / 6-31+\mathrm{G}^{*}\right)($ benzene $)=-1806.21321353214$

$\mathrm{E}\left(\mathrm{M} 06-2 \mathrm{X} / 6-311+\mathrm{G}^{* *}\right)=-1805.80899716148$

$\mathrm{G}\left(\mathrm{M} 06-2 \mathrm{X} / 6-311+\mathrm{G}^{* *}\right)($ benzene $)=-1805.357147292$

$\begin{array}{lccc}\text { C1 } & 3.0255211621 & 0.1104605001 & -1.8357374401 \\ \text { C2 } & 1.6464267277 & -0.1899710756 & -1.8062979290 \\ \text { C3 } & 2.9512746103 & 1.9483022282 & -0.1542769448 \\ \text { C4 } & 0.9996159342 & -0.6955341298 & -2.9383808903 \\ \text { C5 } & 1.5619558469 & 1.8854974418 & 0.0890541071 \\ \text { C6 } & 3.6136290473 & 3.1681457075 & -0.0246091224 \\ \text { C7 } & 2.9013797903 & 4.3154980222 & 0.3450380301 \\ \text { C8 } & 1.5267844445 & 4.2464582674 & 0.5839952936 \\ \text { C9 } & 3.4705955884 & -0.3795221166 & 0.5628746450 \\ \text { C10 } & 2.1480883224 & -0.7393560395 & 0.9022559573 \\ \text { C11 } & 1.7364763997 & -0.8972600928 & -4.1099797523 \\ \text { C12 } & 3.1012103215 & -0.6022974932 & -4.1458913152 \\ \text { C13 } & 0.8468457188 & 3.0309708507 & 0.4546654426 \\ \text { C14 } & 3.7467398666 & -0.0991081623 & -3.0100645968 \\ \text { C15 } & 1.8966829363 & -1.6722741562 & 1.9133423311 \\ \text { C16 } & 2.9761744019 & -2.2554574880 & 2.5848865271 \\ \text { C17 } & 4.2869410839 & -1.9054261453 & 2.2530009692 \\ \text { C18 } & 4.5355434102 & -0.9676461232 & 1.2434632519 \\ \text { H19 } & -0.0546097492 & -0.9435548047 & -2.9204266303 \\ \text { H20 } & 4.6837737494 & 3.2244868662 & -0.2108477068 \\ \text { H21 } & 3.4232436288 & 5.2638842976 & 0.4444339662 \\ \text { H22 } & 3.6680169346 & -0.7651438595 & -5.0591439304 \\ \text { H23 } & -0.2217598659 & 2.9955047915 & 0.6278420773 \\ \text { H24 } & 4.8098649573 & 0.1286813182 & -3.0401906814 \\ \text { H25 } & 0.8850084411 & -1.9414901846 & 2.1918725965 \\ \text { H26 } & 5.1207155454 & -2.3611755869 & 2.7810438140 \\ \text { H27 } & 5.5571844487 & -0.6962679377 & 0.9873396680\end{array}$




$\begin{array}{lrrr}\text { P28 } & 0.9543917549 & 0.1719859894 & -0.1481099980 \\ \text { C29 } & 3.6625050801 & 0.6519440339 & -0.5529914429 \\ \text { H30 } & 4.7264187658 & 0.8402910734 & -0.7127527867 \\ \text { C31 } & -1.1193910508 & -0.3933981811 & 1.6476426435 \\ \text { C32 } & -1.9036608713 & -1.4626971344 & 2.1037207772 \\ \text { C33 } & -0.5763198953 & 0.4878037884 & 2.5987466755 \\ \text { C34 } & -2.1216239402 & -1.6554023004 & 3.4727160582 \\ \text { C35 } & -0.7857024573 & 0.2913220426 & 3.9639473828 \\ \text { C36 } & -1.5589471386 & -0.7857718794 & 4.4088406164 \\ \text { C37 } & -1.2968289465 & -1.4080561038 & -0.6944054387 \\ \text { C38 } & -2.4578848745 & -1.4183128402 & -1.4807640939 \\ \text { C39 } & -0.5266144325 & -2.5818138527 & -0.6280065113 \\ \text { C40 } & -2.8253578460 & -2.5634808809 & -2.1961533911 \\ \text { C41 } & -0.8871725680 & -3.7209647578 & -1.3477666114 \\ \text { C42 } & -2.0396481924 & -3.7158712967 & -2.1397117486 \\ \text { C43 } & -1.6748831596 & 1.1001262374 & -0.3589223763 \\ \text { C44 } & -2.6906392858 & 1.6731817249 & 0.4194671180 \\ \text { C45 } & -1.4068061322 & 1.6577457956 & -1.6211878128 \\ \text { C46 } & -3.4033610888 & 2.7850378370 & -0.0442805177 \\ \text { C47 } & -2.1103024538 & 2.7712020088 & -2.0802703899 \\ \text { C48 } & -3.1126770747 & 3.3436179466 & -1.2905243719 \\ \text { C49 } & -0.9146280495 & -0.1559126117 & 0.1316529899 \\ \text { H50 } & 2.7859837589 & -2.9810987655 & 3.3713834332 \\ \text { H51 } & 1.2383786108 & -1.2910020358 & -4.9921074507 \\ \text { H52 } & 0.9750241399 & 5.1387800062 & 0.8675585047 \\ \text { H53 } & -3.6634626464 & 4.2108866621 & -1.6461600019 \\ \text { H54 } & -1.8744463857 & 3.1878344363 & -3.0565205155 \\ \text { H55 } & -4.1881766154 & 3.2107971989 & 0.5763696096 \\ \text { H56 } & -2.9392493845 & 1.2572538401 & 1.3892484729 \\ \text { H57 } & -0.6462467655 & 1.2292242594 & -2.2655233340 \\ \text { H58 } & -3.0880313079 & -0.5383884491 & -1.5400006767 \\ \text { H59 } & -3.7311353341 & -2.5480146598 & -2.7975625623 \\ \text { H60 } & -2.3235842928 & -4.6025413891 & -2.7012686587 \\ \text { H61 } & -2.3553996076 & -2.1512823858 & 1.3986661878 \\ \text { H62 } & -0.2672455555 & -4.6120360574 & -1.2865210435 \\ \text { H63 } & 0.3656635102 & -2.6240974273 & -0.0127785180 \\ \text { H64 } & 0.0174032535 & 1.3409340009 & 2.2876269006 \\ \text { H65 } & -0.3452738513 & 0.9827219475 & 4.6781725416 \\ \text { H66 } & -1.7247552056 & -0.9407333680 & 5.4721269187 \\ \text { H67 } & -2.7354318468 & -2.4905806948 & 3.8015529929 \\ & & & \end{array}$

\section{He-ring}

$\mathrm{E}\left(\mathrm{BP} 86 / 6-31 \mathrm{G}^{*}\right)($ toluene $)=-23.15522221939$

$\begin{array}{lrrr}\text { He1 } & 2.4999755124 & 0.0000000000 & 0.0000000000 \\ \text { He2 } & -2.4999755124 & 0.0000000000 & 0.0000000000 \\ \text { He3 } & 0.0000000000 & 2.4999755124 & 0.0000000000 \\ \text { He4 } & 0.0000000000 & -2.4999755124 & 0.0000000000 \\ \text { He5 } & 1.7677496376 & 1.7677496376 & 0.0000000000\end{array}$




$\begin{array}{lrrr}\text { He6 } & -1.7677496376 & -1.7677496376 & 0.0000000000 \\ \text { He7 } & -1.7677496376 & 1.7677496376 & 0.0000000000 \\ \text { He8 } & 1.7677496376 & -1.7677496376 & 0.0000000000\end{array}$

\section{P-trip-Hes-ring complex}

$\begin{array}{lccc}\text { E(BP86/6-31G*)(toluene) }=-1096.36283945173 & \\ & & & \\ \text { He1 } & 3.7735999868 \# & 3.4439999914 \# & 5.4275999957 \# \\ \text { He2 } & 3.7668000200 \# & 1.0683000215 \# & 9.8272000084 \# \\ \text { He3 } & 5.2222999744 \# & 0.4646000077 \# & 6.6623000067 \# \\ \text { He4 } & 2.3179999802 \# & 4.0476999924 \# & 8.5925999941 \# \\ \text { He5 } & 4.7994000077 \# & 1.8292000049 \# & 5.3895000026 \# \\ \text { He6 } & 2.7423000070 \# & 2.6840000045 \# & 9.8658000008 \# \\ \text { He7 } & 4.7945999769 \# & 0.1493999800 \# & 8.5003999912 \# \\ \text { He8 } & 2.7457000127 \# & 4.3629000000 \# & 6.7544000168 \# \\ \text { X9 } & 3.7702000000 \# & 2.256100000 \# & 7.6274000000 \# \\ \text { C10 } & -1.7389037679 & 1.4132074549 & 5.0295880407 \\ \text { C11 } & -1.7873632337 & 2.7417485042 & 4.5762908083 \\ \text { C12 } & -0.7148324818 & 3.6034085873 & 4.8369688159 \\ \text { C13 } & 0.4028516583 & 3.1449442447 & 5.5571846845 \\ \text { C14 } & 0.4577421720 & 1.8223612545 & 6.0246298172 \\ \text { C15 } & 1.9736947064 & -0.4352966653 & 5.8746224704 \\ \text { C16 } & 3.1413898915 & -0.9294753495 & 5.2710470546 \\ \text { C17 } & 3.0941900736 & -2.0650441237 & 4.4426502025 \\ \text { C18 } & 1.8773995192 & -2.7167809935 & 4.2070867697 \\ \text { C19 } & 0.7000032574 & -2.2224763690 & 4.7914568400 \\ \text { C20 } & 0.7457090071 & -1.0886342639 & 5.6132297533 \\ \text { C21 } & -0.6249379782 & 0.9548148420 & 5.7451632121 \\ \text { H22 } & -2.5703873333 & 0.7276721939 & 4.8219438982 \\ \text { H23 } & -2.6601688739 & 3.0973137895 & 4.0165197136 \\ \text { H24 } & -0.7402920635 & 4.6395182049 & 4.4798248830 \\ \text { H25 } & 1.2290845266 & 3.8302693578 & 5.7407044140 \\ \text { H26 } & 4.0970304030 & -0.4335066288 & 5.4310832029 \\ \text { H27 } & 4.0168484678 & -2.4357750984 & 3.9811513714 \\ \text { H28 } & 1.8390964427 & -3.6036777400 & 3.5640650624 \\ \text { H29 } & -0.2613339066 & -2.7168655033 & 4.6022626855 \\ \text { C30 } & -1.0293100419 & -1.0480378146 & 10.0036763201 \\ \text { C31 } & 0.0602025850 & -0.3344674844 & 10.5184018634 \\ \text { C32 } & 0.9559347425 & 0.3144882337 & 9.6503198700 \\ \text { C33 } & 0.7733243723 & 0.2564775368 & 8.2588685027 \\ \text { C34 } & -0.3342784905 & -0.4614483009 & 7.7493309769 \\ \text { C35 } & -1.2261834608 & -1.1090027027 & 8.6148824724 \\ \text { H36 } & -1.7281154182 & -1.5554368192 & 10.6787581557 \\ \text { H37 } & 0.2204478099 & -0.2775081264 & 11.6013360664 \\ \text { H38 } & 1.7926082834 & 0.8656659435 & 10.0751207547 \\ \text { H39 } & -2.0790032416 & -1.6616796449 & 8.2005418580 \\ \text { P40 } & 1.9136000342 \# & 1.0922999977 \# & 6.9972999837 \# \\ \text { C41 } & -0.5075813812 & -0.4848063029 & 6.2367954877 \\ \text { H42 } & -1.4023343601 & -1.0668598392 & 5.9551104584\end{array}$


$\mathrm{PPh}_{3}-\mathrm{He}_{8}$-ring complex

$\mathrm{E}\left(\mathrm{BP} 86 / 6-31 \mathrm{G}^{*}\right)($ toluene $)=-1059.43965986558$

\begin{tabular}{|c|c|c|c|}
\hline He1 & $-0.2615000858 \#$ & $-1.5215000030 \#$ & -1.9663999904\# \\
\hline $\mathrm{He} 2$ & $0.2615999904 \#$ & $1.5214999674 \#$ & 1.9662999398\# \\
\hline $\mathrm{He} 3$ & $0.2005999376 \#$ & -1.9836999803\# & $1.5082000030 \#$ \\
\hline $\mathrm{He} 4$ & $-0.2004999977 \#$ & $1.9835999905 \#$ & $-1.5082999481 \#$ \\
\hline He5 & $-0.0431000230 \#$ & $-2.4785999656 \#$ & $-0.3239999712 \#$ \\
\hline He6 & $0.0431999170 \#$ & $2.4785000351 \#$ & $0.3239000216 \#$ \\
\hline $\mathrm{He} 7$ & $0.3268000441 \#$ & $-0.3269000000 \#$ & $2.4567999941 \#$ \\
\hline $\mathrm{He} 8$ & $-0.3266999495 \#$ & $0.3268000000 \#$ & $-2.4569000067 \#$ \\
\hline X9 & $0.0001000000 \#$ & $0.0000000000 \#$ & $0.0000000000 \#$ \\
\hline $\mathrm{C} 10$ & -2.9784806379 & -0.4274621367 & 1.9743650094 \\
\hline C11 & -4.0394896502 & -1.3378771531 & 2.1651706445 \\
\hline C12 & -2.4066260051 & 0.1784874309 & 3.1166877597 \\
\hline C13 & -4.5047491045 & -1.6395456446 & 3.4546005729 \\
\hline $\mathrm{C} 14$ & -2.8822766929 & -0.1115048062 & 4.4039732565 \\
\hline $\mathrm{C} 15$ & -3.9298359396 & -1.0285816141 & 4.5786743924 \\
\hline $\mathrm{C} 16$ & -3.2640762735 & -1.1345105552 & -0.7965774901 \\
\hline C17 & -4.2986578785 & -0.7096458310 & -1.6558839207 \\
\hline C18 & -2.9376650955 & -2.5104926416 & -0.7768249703 \\
\hline C19 & -4.9713034552 & -1.6266498123 & -2.4797354854 \\
\hline $\mathrm{C} 20$ & -3.6199153349 & -3.4278737434 & -1.5876613033 \\
\hline $\mathrm{C} 21$ & -4.6355725573 & -2.9876666939 & -2.4508357880 \\
\hline $\mathrm{C} 22$ & -3.0712221339 & 1.6532718015 & -0.0266113689 \\
\hline $\mathrm{C} 23$ & -3.7543740080 & 2.3963639711 & 0.9587314442 \\
\hline $\mathrm{C} 24$ & -2.9311086352 & 2.2266735270 & -1.3123943119 \\
\hline $\mathrm{C} 25$ & -4.2731786609 & 3.6691418971 & 0.6706976754 \\
\hline $\mathrm{C} 26$ & -3.4628049826 & 3.4910385452 & -1.6035840062 \\
\hline $\mathrm{C} 27$ & -4.1323614066 & 4.2228173560 & -0.6099707335 \\
\hline P28 & $-2.2600998330 \#$ & $-0.0001000440 \#$ & $0.3004999578 \#$ \\
\hline H29 & -2.4094349107 & 1.6750378083 & -2.1011473351 \\
\hline $\mathrm{H} 30$ & -3.3464397472 & 3.9072500371 & -2.6111251590 \\
\hline H31 & -4.5405384662 & 5.2148365058 & -0.8333653395 \\
\hline H32 & -4.7995392231 & 4.2254719739 & 1.4552346776 \\
\hline H33 & -3.8893504108 & 1.9780411418 & 1.9611114718 \\
\hline H34 & -1.5865690698 & 0.8929289450 & 3.0005597965 \\
\hline H35 & -2.4254031424 & 0.3760348221 & 5.2730592835 \\
\hline H36 & -4.2955549841 & -1.2647952810 & 5.5844086863 \\
\hline H37 & -5.3288863068 & -2.3520802271 & 3.5776291578 \\
\hline H38 & -4.5084874840 & -1.8161668387 & 1.2998471181 \\
\hline H39 & -2.1507922630 & -2.8725697128 & -0.1086409657 \\
\hline $\mathrm{H} 40$ & -3.3511306790 & -4.4898965962 & -1.5483848854 \\
\hline H41 & -5.1629372822 & -3.7017074117 & -3.0934827006 \\
\hline $\mathrm{H} 42$ & -5.7706638143 & -1.2713241147 & -3.1406744301 \\
\hline H43 & -4.5885583728 & 0.3453046121 & -1.6811964826 \\
\hline
\end{tabular}




\section{References}

1. Chen, L. Y.; Ren, P.; Carrow, B. P. Tri(1-adamantyl)phosphine: Expanding the Boundary of ElectronReleasing Character Available to Organophosphorus Compounds. J. Am. Chem. Soc. 2016, 138, 6392-6395.

2. Serron, S.; Huang, J.; Nolan, S. P. Solution Thermochemical Study of Tertiary Phosphine Ligand Substitution Reactions in the $\mathrm{Rh}(\mathrm{acac})(\mathrm{CO})\left(\mathrm{PR}_{3}\right)$ System. Organometallics 1998, 17, 534-539.

3. Durand, D. J.; Fey, N. Computational Ligand Descriptors for Catalyst Design. Chem. Rev. 2019, 119, 6561-6594.

4. a) Mayr, H.; Ofial, A. R. Philicities, Fugalities, and Equilibrium Constants. Acc. Chem. Res. 2016, 49, 952-965. b) Mayr, H.; Ammer, J.; Baidya, M.; Maji, B.; Nigst, T. A.; Ofial, A. R.; Singer, T. Scales of Lewis basicities toward C-centered Lewis acids (carbocations). J. Am. Chem. Soc. 2015, 137, 2580-2599. c) Follet, E.; Mayer, P.; Stephenson, D. S.; Ofial, A. R.; Berionni, G. Reactivity-Tuning in Frustrated Lewis Pairs: Nucleophilicity and Lewis Basicity of Sterically Hindered Phosphines. Chem. Eur. J. 2017, 23, 7422 7427.

5. IGMPlot is a software which enables a graphical visualization of NCI in molecules; each NCI being depicted by a green surface. See: a) Lefebvre, C.; Rubez, G.; Khartabil, H.; Boisson, J.-C.; Contreras-García, J.; Hénon, E. Accurately Extracting the Signature of Intermolecular Interactions Present in the NCI Plot of the Reduced Density Gradient Versus Electron Density. Phys. Chem. Chem. Phys. 2017, 19, 17928-17936. b) Lefebvre, C.; Khartabil, H.; Boisson, J.-C.; Contreras-García, J.; Piquemal, J.-P.; Hénon, E. Independent Gradient Model: a New Approach for Probing Strong and Weak Interactions in Molecules from Wave Function Calculations. Chem Phys Chem 2018, 19, 724-735.

6. Humphrey, W.; Dalke, A.; Schulten, K. VMD: visual molecular dynamics. J. Molec. Graphics 1996, 14, 33-38.

7. Poë, A. J.; Farrar, D. H.; Zheng, Y. Systematic Kinetics of High Nuclearity Metal Carbonyl Clusters. Associative Substitution Reactions of $\mathrm{Ru}_{6} \mathrm{C}(\mathrm{CO})_{17}$ with P-donor Nucleophiles. J. Am. Chem. Soc. 1992, 114 , $5146-5152$. 